\title{
GAMIFICATION: THE MAGIC CIRCLE OF TECHNOLOGY
}

by

\author{
Lyuba Encheva
}

Master of Arts, Ryerson University and York University, Toronto, Ontario, Canada 2009 Bachelor of Arts, University of Toronto, Toronto, Ontario, Canada 2003

A dissertation presented to Ryerson University and York University in partial fulfillment of the requirements for the degree of Doctor of Philosophy in the program of Communication and Culture

Toronto, Ontario, Canada 2017

(c) Lyuba Encheva 2017 


\section{AUTHOR'S DECLARATION FOR ELECTRONIC SUBMISSION OF A DISSERTATION}

I hereby declare that I am the sole author of this dissertation. This is a true copy of the dissertation, including any required final revisions, as accepted by my examiners.

I authorize Ryerson University to lend this dissertation to other institutions or individuals for the purpose of scholarly research.

I further authorize Ryerson University to reproduce this dissertation by photocopying or by other means, in total or in part, at the request of other institutions or individuals for the purpose of scholarly research.

I understand that my dissertation may be made electronically available to the public. 


\author{
ABSTRACT \\ Gamification: The Magic Circle of Technology \\ Lyuba Encheva \\ Doctor of Philosophy \\ Graduate Program in Communication and Culture \\ Ryerson University and York University 2017
}

In recent years gamification has emerged as a design trend in customer relationship management, marketing, education and governance. It promotes the use of game design principles in the organization of every day environments, tasks and interactions. As an offspring of advanced communication technologies, gamification relies on the unhindered use of networked devices that transforms every experience into a user experience. Borrowing on the ubiquitous popularity of video games, the premise of gamification is the technologically enabled relationship between virtual causes and real-life effects, and its promise - a mutually beneficial coordination of corporate and personal interest.

This dissertation outlines the socio-political implications of the concept of gamification through a critical examination of its content and intended meanings. The unpacking of gamification as an aspiration and a worldview reveals that as soon as we take for granted the equality of the sign and the signified, we also accept that life experiences do not exceed the signs we use to describe them. Therefore, to play life as a game, as gamifiers urge, is to live life by design.

The definition I coin considers gamification from the perspective of political consequences, rather than practical application and mechanics. I work towards this 
definition by focusing on the rhetoric of gamification as an expressed intention that constructs motives and renegotiates beliefs. Hence, the theoretical model I apply draws on the work of two major theorists. American rhetorician and philosopher Kenneth Burke offers a theoretical apparatus for the study of the form and rhetorical devices of addressed messages. French semiotician and social theorist, Jean Baudrillard, informs the deconstruction of the claims gamification makes. The treatment of language as intention and action that is necessarily subjective and interested, offers a liminal stand-point from where the vision of a gamified world can be seen as an ideology which normalises itself by rhetorical means. Thus, I propose that the concept of gamification, whether applied in practice or not, is a political act. It constructs an ideology that seeks to reconcile the myth of the sacrosanct freedom of the Western individual with the constant imposition of corporate and government demands for compliance, accountability and efficiency. 


\section{ACKNOWLEDGEMENTS}

This dissertation was brought to completion with the generous support and contribution of a great number of people. First and foremost, I would like to express my deep gratitude to my main research supervisor and mentor Isabel Pedersen for the careful guidance, enthusiastic encouragement and useful critiques of this work. Her personal investment and care about the success of this project made me feel as part of a winning team.

My heartfelt thanks to Steven Bailey and John Caruana for their close attention to my writing and timely criticism which pointed the way to a more sophisticated way of thinking and constructing arguments.

I am also particularly grateful to Paul Moore and Jo Ann Mackie for finding the solution to every administrative challenge I presented them with and for tirelessly cheering me on to the finish line.

This work would have not been completed without my colleagues and friends Temenuga, Iona, Lilia and Christine who alleviated the loneliness of dissertation writing with cheerful interventions, shared inspiration and lively discussions of ideas. Most of all, thank you

Boris for seeing the point in my text even when I did not, and Elena for the indispensable help with word-processing and formatting.

Finally, I wish to thank my parents and sister for the loving support and kind reminders that life continues even after the dissertation. 


\section{TABLE OF CONTENTS}

Abstract iii

Acknowledgements $\quad$ V

$\begin{array}{ll}\text { Introduction } & 1\end{array}$

1. Previous Scholarship on Gamification 6

2. Theoretical Resources and Method 15

Chapter 1: Burke and Baudrillard as Informants of the Critique of Gamification 25

I. Kenneth Burke: 28

1. Elements of the Pentad 29

2. Pentadic Ratios 30

3. Substance 32

4. Rhetoric 36

5. Logology 38

II. Jean Baudrillard: $\quad 39$

1. The Link between Symbolic Action and Political Economy 41

2. Stages of Simulation 45

Chapter 2: $\quad$ The Grammar and Rhetoric of Gamification $\quad 50$

I. Utilitarian Gamifiers

1. Hugos: Enterprise Games 53 
2. Penenberg: Play at Work 64

3. The Act of Persuasion of Utilitarian Gamifiers 72

II. Humanistic Gamifiers 73

1. McGonigal: Reality is Broken 74

III. Rhetoric on Gamification $\quad 85$

1. Bogost: How to Do Things with Games 88

Chapter 3: $\quad$ Pymetrics as an Integral Reality Artefact $\quad 100$

$\begin{array}{ll}\text { 1. Pymetrics in Context } & 104\end{array}$

2. The Science behind Pymetrics' Product 105

3. The Treatment of the Subject or Pymetrics as a Self-relation 111

4. The Pymetrics' Subject in Time 122

$\begin{array}{lll}\text { Chapter 4: } & \text { Flow, Freedom and the Gamified Sublime } & 127\end{array}$

1. The Sublime Effects of 'Flow' 131

2. Flow as Being in Control 134

3. Flow as Self-Preservation $\quad 140$

4. Bataille on Eroticism and Sovereignty 145

5. Flow as the Gamified Sublime 152

$\begin{array}{ll}\text { Conclusion } & 157\end{array}$

$\begin{array}{lr}\text { Bibliography } & 168\end{array}$ 


\section{INTRODUCTION}

"The realms of space invaders and spreadsheets are merging: are you ready to play your entire life as a game?"(Campbell, M. 2011)

The quote above is the opening sentence of MacGregor Campbell's article "Game on:

When work becomes play" published in the New Scientist magazine on January 12, 2011.

I have chosen it because it captures in a nutshell the aspirations of a growing trend in user experience design which seeks to introduce the "fun" of games in non-game contexts.

2011 is the year when "gamification" becomes a buzz word for marketers, interface designers, educators, human resource professionals and corporate managers who see it as a promising new strategy for motivation of desired behaviours. Coming to prominence around 2009, up to present day 2017, the gamification movement has generated a wave of book publications, government funded research in $\mathrm{Canada}^{1}$, US and Europe ${ }^{2}$, and regular

\footnotetext{
${ }^{1}$ IMMERSe: the Interactive and Multi-Modal Experience Research Syndicate based at the University of Waterloo has been awarded a partnership grant by the Social Sciences and Humanities Research Council of Canada (SSHRC) to establish a research network for the study of game interactions, gameplay mechanics, multimodality in the game, serious games and game-based learning, and games that change behaviour.

${ }^{2}$ Advanced digital gaming/gamification technologies was one of the topics included in the call for research ICT 2014 - Information and Communications Technologies made by the European Commission in 2013, with a total call budget of EU 658,500,000 (http://ec.europa.eu/research/participants/portal/ desktop/en/opportunities/h2020/topics/90-ict-21-2014.html\#tab2)
} 
following by a number of professional and academic conferences ${ }^{3}$. Companies, which create or use gamification applications are also multiplying. Some of the more established ones are Bunchball, Badgeville, Salesforce, IBM, Samsung.

This dissertation interrogates gamification as a rhetorical phenomenon, design concept and social practice. Through my analysis of its instantiations, I question the popularity of gamification, its relevance to the current cultural landscape and call for a serious examination of its implications. The suggestion that game design principles can be applied to the organization of every-day life brings into focus the increasing presence and social acceptance of pre-designed user experiences. Gamification, as an offspring of advanced communication and entertainment technologies, relies on the uninterrupted use of networked devices that necessarily transform every experience into a user experience with pre-determined possibilities and constraints. In this context Campbell's light-hearted invitation to play our entire lives as a game becomes problematic. Game theorists already interrogate the complex relationship between free choice and addiction, fun and control in game environments. But what happens when it is suggested that life itself is a game? Once the gamification of the everyday becomes the set goal of innovators, we have to begin questioning the said need to mix real and imaginary experiences. What are the benefits we expect, the problems that we hope to solve, or the problems that might arise due to the intentional convergence of the physical and virtual realms?

Presently, games like Pokémon Go claim to keep us physically active by enticing us to chase augmented reality Pokémons. Location-based applications like Four Square increase our 'popularity score' and sense of connectedness in return of continuous

\footnotetext{
${ }^{3}$ GSummit, San Francisco and New York (2010-2015); CHI PLAY: The ACM SIGCHI Annual Symposium on Computer-Human Interaction in Play (Toronto 2014, London 2015); Gamification World Congress (Spain, 2012, 2015)
} 
disclosure of our daily routes and activities. Gamified applications like Habitica and Chore Wars improve our productivity by making us compete against the cartooned avatars of team members and by awarding points for the completion of routine tasks. Yet, all of these games, gamified applications and social media platforms select narrow targets and produce limited effects.

What applied gamification would look like when perfected and ubiquitous is well illustrated by Ender's Game, a science fiction film released in 2013 based on the novel of the same name by Orson Scott Card. It describes a time in the future when computer simulations and gamification are the main means for human resource management and social control. As humanity prepares for a counter attack on an alien civilization, and the most elite warriors are children who excel at playing video games. Among them, Ender a child prodigy with an aptitude for quick adaptation to simulated environments and strategic thinking, is singled out by the leaders of the operation and put through a series of training tests. The final test before Ender's graduation is a large scale battle simulation which he leads from a commanding pool in front of huge video screens. When the testing battle finally ends with the triumph of Ender and his team, the screens are restarted to reveal that the battle was real and the homeworld of the alien civilization is already destroyed. Ender is shocked by the revelation and struggling to come to terms with the fact that he has just annihilated an entire world by playing and winning a "game". The protagonist's reaction of anger and regret at his supposed victory demonstrates the contradictory effects of gamified experience. His performance may have been enhanced by the calm, strategic thinking one can muster when unaware of the high "stakes of the game". Yet, the sense of empowerment he gets through the masterful execution of the 
task at hand is immediately removed by the realization that the final outcome is not the one he anticipated and intended. The game he played for the sake of excellence, turns out to be the unannounced means to someone else's end. Ender's freedom of action is reversed by the impossibility of free choice in the world of Orson Scott Card where simulation and reality seamlessly overlap. His novel (and the movie based on it) suggests that the value and utility of simulation are contingent on the quality of the orchestrated deception. As it appears, the 'game' part of gamification does not refer to a game, but to a simulation that bridges perilously the boundary between imaginary and real. Metaphorically speaking, gamification claims the problematic roles of both "the cheat" and "the spoil-sport" as described by Huizinga in Homo Ludens. Like the cheat, it "pretends to be playing the game, on the face of it still acknowledge(ing) the magic circle" (Huizinga ,1970, p.11). Yet, like the spoil-sport, it also "shatters the play-world itself" by disrespecting its conventions (Huizinga, 1970, p.11). Gamification is disruptive for Ender, or the gamified subject at large, because it is neither game, nor reality, but a simulation that breaks the magic circle of the game by opening it up to real-life consequences. This is a risky manoeuvre, which, according to Huizinga, certainly ruins the game, and according to Baudrillard, threatens the concept of reality altogether.

Scott Card's depiction of the future of perfect simulation and the forthcoming analysis of gamification rhetoric and discourse point toward the same implications.

\footnotetext{
${ }^{4}$ The Dutch historian and anthropologist Johan Huizinga uses the term "magic circle" to describe the intended separation between the play-world and ordinary world, which allows the player space for free experimentation: "All play moves and has its being within a play-ground marked off beforehand either materially or ideally, deliberately or as a matter of course. Just as there is no formal difference between play and ritual, so the "consecrated spot" cannot be formally distinguished from the play-ground. The arena, the card-table, the magic circle, the temple, the stage, the screen, (...) are all in form and function play-grounds, i.e. forbidden spots, isolated, hedged, round, hallowed, within which special rules obtain. All are temporary worlds within the ordinary world, dedicated to the performance of an act apart." (Huizinga, 1970, p.10) Many game theorists adopt Huizinga's term in their discussions of games and video games.
} 
Playing life as a game is a way of relating to the world that invalidates long-standing dualities such as imaginary and real, representation and experience, sign and signified. This state of "virtuality" or "integral reality", as Baudrillard calls the contemporary condition in a number of his later works, makes any differentiation impossible. Not only that, the merging of opposites, the lack of difference or simulated in-difference is intended and purposeful. As soon as we take for granted the equality of the sign and the signified, we also accept that life experiences do not exceed the signs we use to describe them. The unpacking of gamification as an aspiration and a worldview reveals that from the equivalence of lived experience with simulated ones, to the belief that life itself can be designed according to our liking, there is a small step, and we have already taken it. To play life as a game is to live life by design, but whose design?

With this work, I propose that the concept of gamification, whether applied in practice or not, is a political act. It constructs an ideology that seeks to reconcile the myth of the sacrosanct freedom of the Western individual with the constant imposition of corporate and government demands for compliance, accountability and efficiency. The gamified subject is socialized to feel as powerful and ecstatic as a demi-god, while her every move is transformed into the reliable means toward greater and ulterior ends. She is granted the right to enter the deception or the simulation of her choice; and the freedom to resign from freedom for the 'sense' of being in control.

Despite its alleged applicability in various areas of human experience, gamification has not emerged spontaneously as a social practice or a grassroots cultural phenomenon. It is more akin to a design vision, a concept whose pretext is the ubiquitous popularity of video games, its premise is the technologically enabled connection between 
virtual experiences and real-life effects, and its promise - a mutually beneficial coordination of corporate and personal interest. This dissertation revises the sociopolitical implications of the concept of gamification through a critical examination of its content and intended meanings. While I study how gamification speaks, and what it says, I also study its relationship to 'the real' (work), the 'imaginary' (game), and the lived experience of the subject who has to navigate this new space. For the purposes of this investigation, I have formed a theoretical model that draws on the work of two major theorists. American rhetorician and philosopher Kenneth Burke offers a theoretical apparatus for the study of the form and rhetorical devices of addressed messages. French post-structuralist thinker, semiotician and social theorist, Jean Baudrillard, informs the deconstruction of the claims gamification makes. Both authors serve my intention to expose ideological constructions in gamification texts, discourse and practice.

\section{Previous Scholarship on Gamification}

While examples of gamified experiences and products are growing in numbers, they have not been around long enough to be considered an established cultural practice or to be studied thoroughly. For that reason, existing theoretical discourse on gamification builds on scholarly traditions in areas of closest proximity. Thus, if gamification is about finding and enhancing the game-like qualities in everyday things, an investigation into gamification implies an investigation into the phenomenon of play. The nature of play has been an object of exploration for philosophers and cultural theorists for a long time. Some prominent discussions on the subject come from writers like Johan Huizinga (1949), Roger Caillois (1961), Brian Sutton-Smith (1997), and Bernard Suits (1978) all of whom 
seek to determine the significance of play in human life and culture. More immediately still, gamification is affiliated with game studies and game design theory, which emerge as fields of research after the rise of the video game industry in the 1980s. Social scientists and humanities scholars like Sherry Turkle (1995), T. L. Taylor (2006), Henry Jenkins $(2006 \mathrm{a} / \mathrm{b})$ explore the ways gaming affects player identity, the mechanics of multi-player interactions, or the functioning of video games as transmedia storytelling. At the same time, game designers like Chris Crawford (1984/1979), Salen and Zimmerman (2003), Raph Koster (2004) also begin to formulate a theory of game design outlining the principles of successful player engagement and retention. In the long line of people talking about games and play, the transition from gaming to gamification can best be attributed to economists and organizational theorists like Malone (1980), Malone and Lepper (1987), C. Coonradt (1985), Castronova (2006). Rather than being interested in the cultural, social or psychological implications of (video) games, they focus on the practical or economic advantages that can be derived from a widely popular practice. In his 1999 book The Dream Society, Rolf Jensen of the Copenhagen Institute for Futures Studies not only describes the business utility of games, but predicts a future economy and marketplace driven by storytelling and emotions rather than functionality and efficiency. Jensen, thus lays the ground for the commodification of human emotion itself. In this context games are reinterpreted as motivational tools in a variety of real-life settings, but also as safe environments for conducting social experiments and behavioural observations. The theoretical foundation of gamification in psychology is mostly derived from Maslow's hierarchy of essential human needs (1970a, 1970b), and the work of positive psychologist Mihaly Csikszentmihalyi who studies the mechanics of genuinely 
satisfying experiences. In his 1990 book Flow Csikszentmihalyi suggests that our love for active sports and games is due to a desire for deep involvement in challenging, straightforward tasks. On this basis, games and play can be redefined as work, but work properly structured to stimulate full engagement and induce intense, positive experiences.

The idea of work as game, or the gamification of everyday activities is then taken on by a number of authors who find the contemporary cultural context to be a fertile soil for its popularisation. These can be divided in various categories depending on the sphere of application and purpose of gamification they propose, but the important commonality between them is that they are all advocates of gamification and can be credited for the creation of its promotional rhetoric. D. H. Pink (2009), T. Chatfield (2010), J. McGonigal (2011), J. Shell $(2008,2010,2011)$ are some of the proponents of gamification with humanitarian aspirations who believe that games and gamification offer the best tools for the achievement of positive social change, personal well-being, and education. Jane McGonigal, for example, addresses the wider audience with the inspiration of a true game idealist. In her book Reality is Broken released in 2011, she expresses her belief that if games have the ability to keep us engaged and happy, they may be the answer to the creation of a better world:

A good game is a unique way of structuring experience and provoking positive emotion. It is an extremely powerful tool for inspiring participation and motivating hard work. And when this tool is deployed on top of a network, it can inspire and motivate tens, hundreds, thousands, or millions of people at a time. (McGonigal, J. 2011, pp.33-34)

Sharing her optimism Koster, Shell and Pink talk about "edutainment" (Koster, 2004), the "pleasure revolution" (Shell, 2011), and the capacity of games to satisfy the innate needs of human beings "to be autonomous, self-determined, and connected to one another" (Pink, 2009, p.71). 
At the other end of the spectrum, authors like D. Edery and E. Mollick (2009), B. Reeves and J. L. Read (2009), Gabe Zichermann (2011), M. Hugos (2012), Penenberg (2013) are promoters of gamification with utilitarian aspirations, in as much as they conceive of games and gamification as the means to the optimisation of business relations and economic growth. Their rhetoric, although still in support of human well-being and progress, more conspicuously normalizes an hierarchy of values where a person's positive experiences and emotions are subordinated to material gain.

The value system of promotional gamification rhetoric is consistent with that of applied gamification rhetoric. The latter one is produced by actual companies like Gamify Inc., Seriosity, Bunchball, Lithium, Pymetrics, and many others selling gamified services and applications or gamification expertise. This group of gamification experts and practitioners is primarily focused on what can be achieved by means of gamification and how. Thus, Lithium - a company that creates gamified solutions for social media management and customer service, urges its prospective clients with the imperative: "Drive the Right Customer Behaviors with Gamification!" ${ }^{5}$ Similarly, Bunchball, one of the first gamification companies promises on its website: "Now you can harness the power of gamification to engage your audience!" ${ }^{6}$. As a customer relationship expert, Kelly Liyakasa urges: "If you want better results from employees and customers, let them play" (Liyakasa, 2012). The title page of her article also states: "The things that make games so compelling "can equally make employees, partners, [and] customers addicted to your $b 2 b$ or $b 2 c$ offering." This is a way of speaking which transforms the inspired visions of gamification advocates into concrete, sellable method and practice.

\footnotetext{
${ }^{5}$ Lithium, Resources: "Drive the Right Customer Behaviors with Gamification!" (white paper) (http://pages.lithium.com/gamification-spectrum.html), (retrieved Nov. 10, 2016)

${ }^{6}$ (http://www.bunchball.com/, (retrieved Nov.10, 2016))
} 
The potential use of games in non-game environments is now acknowledged by the Social Sciences and Humanities Research Council of Canada, which in 2013 awarded a 2.5 million grant to IMMERSe and the Game Institute at the University of Waterloo to study game interactions, serious games, game-based learning, and games that change behaviour. The Games Institute, for example, participates in the GET FACTS project by gamifying allergy awareness through the production of table-top games that are capable of educating children and the general public of common allergens found in basic dishes. Peer Doc is another project which seeks to implement a gamification structure for an academic writing and mentorship site to further motivate and engage users.

In addition to the promotional gamification rhetoric and projects on applied gamification, there have been some involved theoretical discussions on the notion of gamification which have sought to define and situate it in a wider social and cultural context. These shape the theoretical discourse on gamification. Thus, Deterding, Dixon, Khaled and Nacke (2011a) are the first to coin a formal and comprehensive definition of gamification. According to them, the term "gamification" means: "using game design elements in non-game contexts". They emphasize that gamification is related to games (which have set rules), rather than free play or improvisation; it uses design elements rather than "full-fledged" games; and applies these in non-game contexts for other than pure entertainment purposes. The distinctions drawn by this definition legitimate the notion and outline its general parameter.

From a more critical stance, Ian Bogost, a game designer and media studies professor, dubs gamification with the infamous term "exploitationware" to expose the misuse of "games as a mod marketing miracle " (Bogost, 2011a). Later, in his book How 
to Do Things with Videogames (2011b) Bogost also treats games as a new medium that can be effectively applied in various real-life contexts. The latter is a sequel to his 2007 book called Persuasive Games, where Bogost claims that videogames open a new domain for persuasion that he names "procedural rhetoric". Bogost's "procedural rhetoric" draws on the Aristotelian model of effective oratory that moves though established steps of argumentation and familiar figures of speech. Additionally, based on Kenneth Burke, the author also claims that the symbolic systems we use are not necessarily and strictly verbal, therefore rhetoric can be understood to facilitate and describe human action in general. As he inserts computational processes within the rhetorical paradigm, Bogost defines procedural rhetoric as the "art of persuasion through rule-based representations and interactions" (2007, ix). Although Bogost is careful to maintain the distinction between persuasive games and gamification as a strategy of persuasion, his notion of procedural rhetoric points towards the applicability of games to purposes that exceed play. The conceptualization of games as a medium is a theme that permeates Bogost's earlier body of work. Ironically, the strong case he makes on the persuasive power of games may be seen to contribute, at least indirectly, to the argument of gamification. Bogost's innovative outlook on games as argumentative structures produces a method that is specifically tailored to assess the value and impact of games as cultural artefacts. While it effectively illuminates the functioning of procedural rhetoric, Bogost's analytical prism is not applicable to the examination of gamification rhetoric as an attitude.

Still, theorists who are concerned directly with gamification are far and wide between. The majority of in-depth analysis produced by the fields of media, game and cultural studies centers on the cultural significance of games, virtual reality and touches 
on (or can be referred to) gamification only peripherally. Thus for example, Grimes and Feenberg discuss digital games from the perspective of critical theory in order to construct a 'ludification theory" that re-imagines "games as sources of social order" (2012, pp. $25-27)^{7}$. With this, the authors emphasize that play and leisure have not only been assimilated into the rational realms of production and consumption, but games have come to function as institutionalized entities that reproduce "the larger processes of rationalization at work within modern capitalist societies" (2012, p.21). While Grimes and Feenberg take a stab at defining the political function of digital games, the question regarding the role of gamification on the contemporary socio-cultural scene remains open.

Another theorist who studies the impact of digital media and virtuality is Mark Poster $^{8}$. He studies virtualization as a new "mode of information" exchange that "instigates the transformation of existing cultural figures" and "encourages practices, that in turn, serve to construct new types of subjects" (Poster, 2001, pp.3-4). While he recognizes the need for critical examination of the "political culture of new media" (2001, p.4), Poster claims that this would be impossible without specifying the parameters of "virtual" configurations that enable new subject-object relationships (2001, p.18). In defining virtual reality, Poster points out that virtual reality applications "provide substitutes for the real" that allow the conditions of the real to be "tested without the normal risks" (2001, p.129). In these specific cases of doubling, he claims, "technology provides prosthesis for the real in order to better control it" (Poster, 2001, p.129). Poster's discussion on the political implications of the Internet and virtual reality can be extended to gamified interactions as well, but he does not yet address the specific subject-object

\footnotetext{
${ }^{7}$ Grimes, M. Feenberg, A. "Rationalizing Play: A Critical Theory of Digital Gaming", (Re)Inventing the Internet: Critical Case Studies, A. Feenberg and N. Freisen (Eds), Sense Publishers, Rotterdam 2012

${ }^{8}$ Poster, M. What's the Matter with the Internet, University of Minnesota Press, 2001
} 
relationships gamification entails. The taken for granted non-distinction between real and imaginary in gamification is of a different kind than that in virtual reality. If VR is considered a safe practicing environment, simulating but strictly isolated from the rest of life, the gamified transaction intends real consequences.

Following a similar course of investigation, Vincent Mosco claims that narratives of emergent technologies have an important mythological function. Mosco builds on Barthes' (1957/1887) idea that myth is produced when the connotation (culturally specific interpretation) of the sign appears as its denotation - a literal or direct meaning that is devoid of ideology or political implications. By applying Barthes ideas in the context of cyberspace, Mosco explores the next horizon of technological representation and myth formation. According to Mosco, "cyberspace has become the latest icon of the technological sublime, praised for its epochal and transcendent characteristics and demonized for the depths of the evil it can conjure" (2004, p.24). For Mosco the living myths of a society are "a form of reality", a meaning making mechanism, which seeks to reconcile the conflicts and contradictions within the value system of a given time (2004, p.13). They have to be studied both for "what they reveal" in their capacity of dream visions, and "what they conceal" in terms of political interests and mechanisms of power (Mosco, 2004, p.19). By showing how myth as the need for the sublime and political economy work in tandem to drive social practice, the author explains the immediate relationship and contingency between these often polarized forces. While Mosco does not specifically talk about it, gamification too mixes not only the embodied and the virtual, but the mythical and the political. In parallel with his claim that myth "inflects human 
values with ideology" (Mosco, 2004, p.30), I demonstrate what kind of "myth" constitutes the ideological dimension of gamification promotion rhetoric.

Joining the varied choir of voices that speak on gamification, my ambition is not to coin a more precise description of the phenomenon or to prove its existence and viability as a practice. Such an endeavour would imply a revision of the notions of play and games, and the difference in the meaning of traditional vs. technologically enabled games. It would also require the clarification of the difference between a game and reality, and then, the specifics of entities that can or should be classified as partially real or partially game. Gamification also posits the problem of the relationship between humans and technology. I do not explicitly elaborate on this problem here, but I do discuss the great confidence of our culture in the neutrality of science and technology that propels them into the role of arbiters and guarantors of fairness.

The definition of gamification I coin here stems from concern with political implications, rather than application and mechanics. I work towards this definition, not by studying the social practice itself, but by focusing on its rhetoric as an expressed intention that constructs motives and renegotiates beliefs. Gamification rhetoric is a declaration of how we want the world to be, and as such, it functions ideologically to harmonize existing social discords and discontents. Hence, the theoretical model I propose treats the concept of gamification as a tool in its own right, as a probe into a motivated kind of speaking which frames and formulates a life-world. 


\section{Theoretical Resources and Method}

Since this work explores how gamification emerges through discourse, rhetoric, and design concepts, it is most of all a study of the different ways gamification speaks. For that reason, authors who keep in focus the impact of the medium over the message ${ }^{9}$ lend the right analytical tools and critical perspective. The critical model I propose as part of my contribution combines the resources of rhetorical, critical discourse, and semiotic analysis. These analytical techniques emphasize the importance of language and symbolic representation in both the constitution and the interpretation of social phenomena. Therefore, they are particularly helpful in the investigation of an emerging concept, such as gamification, whose presence is better established through its own promotional discourse than in social practices. My analysis combines these approaches in order to better reflect on the different dimensions of the studied problem. Thus, I draw on rhetorical analysis to examine the concept of gamification as a motivated action, on discourse analysis to detail the processes of its affirmation as authoritative knowledge, and on semiotics to unpack the relationship between the constructed sign and conventional belief. My research method relies mainly on the examination of a variety of participatory texts as evidence and expression of the value structures and belief system that shape the present socio-cultural environment. However, at times, it is supplied by my participant perspective as the user of a gamified online application for career development and job search. This is the only way to access the bits of gamification rhetoric specifically

\footnotetext{
${ }^{9}$ The expression I use here "focusing on the impact of the medium over the message" is an unintended pun on Marshal McLuhan's famous aphorism "the medium is the message" (elaborated in his 1964 book, Understanding Media: The Extensions of Man). What I emphasise with this expression is that authors like Burke and Baudrillard remain conscious of the form of representation and the impact of the form over the content of the message. This emphasis may be influenced by McLuhan's idea, but was not a direct reference to him.
} 
addressed to the user and experience first-hand the presence or absence of its intended effects.

Having started as a concept, rather than a phenomenon, gamification is still better established in discourse than in practice. Whenever explained, promoted or contested, the notion remains the subject of a heated debate which is necessarily addressed and rhetorical. For that reason, the study of gamification rhetoric is an even richer source of information on the social implications and significance of the trend than the study of actual gamification applications. My criteria for analysis and definition of rhetoric are based on the American rhetorician Kenneth Burke's extensive writings on the rhetorical function of language. He defines rhetoric as "the use of language as a symbolic means of inducing cooperation in beings that by nature respond to symbols" (Burke, 1969b, 43). Drawing on Burke, I treat gamification rhetoric as a composite notion which includes: rhetoric about gamification - speech or writing that serve to promote and explain it; as well as the rhetoric of gamification - the language used in gamified applications or by those who apply gamification as a design strategy. The distinction between the two kinds of rhetoric, although formally necessary, is not always clear cut, because gamification theorists and promoters are often the ones who apply it.

Building on traditional principles of rhetoric and his view of language as action, Burke formulates the dramatistic pentad as a practical method for the interpretation and allocation of motive. Drawing on Burke's methodology, I treat gamification rhetoric as an action put forth by an actor through selected means and towards particular ends. The resources of Burke's dramatistic and rhetorical analysis help locate the source of that action, as well as catalogue the different types of participants, their roles, purposes and 
hierarchies. Analyzing samples of promotional gamification rhetoric through this prism brings into sharp relief the treatment of the gamified subject, and the ways in which her modes of action and agency are framed by the use of language. Burke's grammatology also unveils the foundational "substances" or definitions which frame the world as 'a given' and allows the examination of the relationship of the gamification attitude with the positivist tendency to ascribe absolute value to scientific knowledge.

As a rhetoric that promotes business applications and design strategies for the future, gamification makes claims that are not sufficiently verified in scientific research nor in practice, and their enthusiastic tone is reminiscent of the utopic optimism of predictive advertising. ${ }^{10}$ Therefore, to study the implications of gamification as a participatory discourse or applied rhetoric I turn to the social commentary of Jean Baudrillard. The radical contribution to postmodernism of this French post-structuralist thinker is a theory of the sign based on the assertion that reality is a construction of representation. The semiotic sign eventually conceals the absence of reality. Eugene Thacker writes that "In Jean Baudrillard's famous formulation, the simulacra is the 'copy without an original'; that is, the logic of simulation proceeds through a paradoxical circuit in which 'the real' is lost at the very moment that it can be perfectly simulated (2001, p.156). Indeed, Baudrillard's writing is drawn upon extensively to provide commentary and criticism of society's increasingly mediated digital lives, the culture and politics of digital space, digital communities and digital identity (Soja 2001, Koch 2005, Lapenta 2011, Constable 2006). The problem that he wants to expose does not lie in any particular

\footnotetext{
${ }^{10}$ Predictive advertising is a term we have coined to describe the adds of technological companies which promote products under development, rather than existing ones.

Encheva, L. and Pedersen, I. (2014) '“One Day...': Google's Project Glass, integral reality and predictive advertising' Continuum: Journal of Media and Cultural Studies Vol. 28 (pp. 235-246)
} 
social practice or phenomenon. The culprit is not technology, the internet, virtuality, or the productivist social order; it is rather an overarching attitude, a way of seeing which overlooks the arbitrariness of the sign in order to sustain its concrete presence and absolute value. In an interview for Le Nouvel Observateur in 2004, Baudrillard points to the close relationship between a society's modes of representation and world-view. He says:

The radical illusion of the world is a problem faced by all great cultures, which they have solved through art and symbolization. What we have invented, in order to support this suffering, is a simulated real, which henceforth supplants the real and is its final solution, a virtual universe from which everything dangerous and negative has been expelled. (Lancelin, 2004)

Baudrillard measures a society by the form and nature of its symbolic actions, or by the quality and type of "reality simulations" it creates. As a simulation of 'the real' and a 'realization' of the imaginary, the concept of gamification is indicative of the way contemporary society handles the "radical illusion of the world" by means of mixing representation with experience. The sample of applied gamification rhetoric I examine demonstrates the consequences of the contemporary movement toward the replacement of "symbolic substance" with perfect simulations. The attitudes and definitions invested in the language of gamifiers are the practical confirmation of Baudrillard's theoretical diagnosis of the contemporary condition.

While Baudrillard provides the theoretical directives for my claims, he does not provide a consistent method of analysis. Therefore, my exploration of applied gamification rhetoric and gamification discourse is also supplied by the means of discourse analysis and semiotics. Gamification owes its popularity to the fact that it breaks from conventions by replacing established oppositions with newly fashioned 
equivalences. However, loud slogans like "fun is the future" or "the future of work is play" $^{11}$ rely on the supposed contrast between work and play as much as on the denial of it. To unpack such contradictions, one should treat gamification as a discourse emerging at the point of convergence of both theoretical and participatory discourses, which frame the notion and determine its cultural interpretation. Since discourse is the starting point of knowledge, power relations and subjectivity, gamification discourse too contributes to the genealogies of familiar notions and the affirmation of specific cultural values as necessary and true. This is the right moment to consider what kinds of knowledge it validates, and what kinds of speaking subjects it produces.

My approach to discourse analysis is informed by the model of Canadian linguist and rhetorician Glen Stillar, who defines discourse as "the social activity through which we make meanings with linguistic and other semiotic resources" (1998, p.12). By extension, the object of discourse analysis is "language activity - actual texts occurring in real contexts" as "instances of meaning-making systems" that embody social activities and relationships (Stillar, 1998, p.20, p.107). The text's meaning depends on the relationship between linguistic signs and patterns, where the chosen application of the linguistic sign acquires significance in opposition to the multiplicity of its potential applications. As Halliday, whom Stillar credits, phrases it: "A text is 'what is meant', selected from the total set of options that constitute what can be meant" (Halliday, 1978, p.109). Furthermore, Stillar clarifies, since the "meaning" of a text is its "function in context", and "the functions of text in context always have social consequences",

11. Gabe Zicherman, "Fun is the Future: Mastering Gamification" GoogleTech Talks, (http://www.youtube.com/watch?v=6O1gNVeaE4g\&list=PL1B1A83D44CDBCA12) Nicole Lazzaro, "The Future of Work is Play", TEDxSoMa (http://www.youtube.com/watch?v=X_3KyV31iqg) 
discourse analysis scrutinizes the organizing, representing and interacting functions of language in order to draw conclusions on the way texts construct social relations, produce social norms and distribute power among groups and individuals. Isabel Pedersen also elaborates on the connection between language and social action as the basis for productive analysis. As she points out in her book Ready to Wear: A Rhetoric of Wearable Computers and Reality-Shifting Media, "Real people act through "symbolic action" and real people are acted upon by "symbol systems" (Pedersen, 2013, p.19). The analytical model I devise here is also informed by Pedersen's use of discourse and rhetorical analysis for the critical discussion of wearable technologies.

By now, it is apparent that the present discussion of gamification draws heavily on the work of theorists who caution against the equation of the sign with its referent, and stress the fact that "the efficacy of the symbol is to be found in its relationship to other terms in the terminology, not in its inherent accuracy in "summing up" the symbolized" (Stillar, 1998, p.82). For Baudrillard the inherent danger born in the confusion of representation and experience consists in the increasing overlap between virtual and physical reality that leads us to an even more advanced stage of simulation than the simulacrum. The kind of symbolic action gamification constitutes is symptomatic of what he calls 'Integral reality', but prior to that, it is the natural consequence of the scientific and universalistic discourse of political economy. Similarly, Kenneth Burke suggests that any philosophical interpretation or system of understanding rests on a selection of takenfor-granted definitions or "substances", which constitute the basic framework of what is. He shows how, the more absolute the value of these definitions becomes, the more philosophical understanding approaches ideological thinking. Perfect "logological" 
systems are perfect ideologies which rest on self-perpetuating paradoxical cycles that demand participation as the only possible recourse. As scholars of discourse and rhetoric Stillar and Pedersen add to the understanding of language as a reality shaping tool. Their work also traces how symbolic action translates into cultural conventions and practices. Thus, the approach I have chosen, is informed by authors and methodologies that rely enough on the connection between the social and the symbolic in order to measure one against the other, and yet continue to be aware and critical of the looming absolutism of the sign.

On this theoretical background, I propose that the concept of gamification not only intends, but takes for granted the equivalence between representation and experience, the sign and the signified. The implications of this equivalence, of playing life as a game, are far reaching. The view that virtuality is indispensable for the "augmentation" of embodied every-day life already makes it "realer" than real - the cause of the real. In the gamified environment the digital output of an individual player becomes the most 'realistic' measure of a person's presence and participation. Thus, as Baudrillard suggests, we observe the transformation of the individual into a data trace that inhabits a virtual realtime, where past and future are condensed into a perpetual present. To imagine that playfulness can be reliably induced by design, is also to treat games as mere composites of mechanics and technology. By extension, fun, desire, spontaneity and mystery can also be abstracted objects of design. They too are reproducible collections of conditions and features that can be transferred from one context to another by digital means. According to the logic of design and gamification, play can be made useful, work can be made entertaining, and "intrinsic" motivation can be externally induced. This way of thinking is 
the natural continuation of scientific objectivism that keeps away from subjective interpretation, but only to give licence to the subject to know with absolute certainty. Likewise, gamification ignores the impact of the player in order to allow for the complete precedence of the designer of the game.

The effects of equating spontaneous and pre-designed experiences, or real and imaginary ones, are also confirmed by the Dramatistic analysis of gamification rhetoric. The treatment of language as intention and action that is necessarily subjective and interested, offers a liminal stand-point from where the vision of a gamified world can be seen as an ideology which normalises itself by rhetorical means. As Burke and Baudrillard agree, it becomes ideological precisely when it loses the distinction between experience and representation or sign and signified. Thus, as a way of thinking, gamification constitutes a legitimate extension of liberal individualist ideology that is also founded on the absolute confidence in scientific truth. Rather than the proclaimed alternative of the neo-liberal subject pressed by material necessity and constructed by relations of power, the gamified subject emerges as its direct offspring. However, this one is not described as an "entrepreneur of himself", who invests in skill development and sells one's labour, but as a subject in free pursuit of one's intrinsic needs and desires that can be met by voluntary submission to the engagement strategies of digital stimulation systems. Gamification seeks to utilize human emotion and use it as the raw material in the production cycle of personal and economic prosperity and happiness. However, rather than designing appealing products, now we design desire itself - we design ourselves.

To contribute to the above argument, each chapter examines the concept of gamification from a different point of view. In the first chapter I describe and reflect on 
the analytical approaches of K. Burke and J. Baudrillard, and their applicability to the investigation of gamification. Regardless of apparent differences in tone and methodology, I demonstrate how their ideas work together to expose the ideological function of gamification rhetoric, and its possible impact as a program of action.

The second chapter focuses on the rhetorical means of promoters and 'experts' of gamification, and the hierarchical relationships they construct. Burke's grammar and rhetoric serve to analyse the texts of several books that promote gamification, as well as Ian Bogost's conceptualization of games as a medium in his 2011 book How to Do Things with Videogames.

In chapter three I apply the tools of discourse and semiotic analysis to examine a sample of applied gamification rhetoric that I source from the website of Pymetrics - a recruitment company which determines best career fit based on neuroscience games. I use the same analytical tools to discuss the texts and structure of the games, my first-hand experience as a player, the assessment I receive and my relationship to it. I conclude that the Pymetrics' way of speaking and treatment of users confirm the transition of Baudrillard's concept of integral reality into social attitude and practice.

Finally, chapter four examines the notion of 'flow' appointed by gamifiers and game designers as an explanation of the motivating power of games. In order to unpack its claims, I examine it on the background of preceding notions such as the classical sublime, Freud's eros and thanatos, and Bataille's eroticism. Here, I argue that the success of 'flow' as a promotional banner for gamification consists in its meaning making capacity as a persuasive periphrasis of sublime affect. The investigation into 'flow' as the 
coveted experience of the gamified subject also serves to evaluate the ideological potential of gamification. 


\section{CHAPTER ONE}

\section{Burke and Baudrillard as Informants of the Critique of Gamification}

As theorists who examine the limits of signification, Kenneth Burke and Jean Baudrillard devise strategies for vigilant observance of the distance between representation and experience. The original confusion of the sign with its referent is expressed in Burke's concept of definition. He uses the term "substance" as a reminder of the fact that definitions seek to capture the "essence" of a thing by describing its boundaries, its foundation, by appointing a core for it in something the thing is not. The initially provisional or interpretative equation of a thing with its "substance" becomes habitual, then automatic and absolute. Referring to the same problem, Baudrillard's word for representation is "simulation". "Simulation", "simulacrum", and "integral reality" are all terms designating the different stages of forgetting that initial condition for the equivalence of the sign and the thing it signifies. More importantly, they are also terms which reflect on the consequences of this forgetting. They indicate that the sign is no longer invested with the intention of the speaker, and its variable subjective interpretation is narrowed down to a singular, "objective" meaning. These basic terms for definition are only the starting points of Burke and Baudrillard's methodologies geared towards 
exposing the inherently ideological nature of language or the make-believe game of representation.

To examine the concept of gamification as a construct of ideological thinking means to be skeptical of it, to be looking for something that does not immediately meet the eye. After Marx, Engels and the Frankfurt school the word 'ideology' designates, not simply a set of beliefs and ideas about the world, but specifically the ideas of the ruling class which also determine the value system and governing principles of a society. As Marx has postulated: "The class which has the means of material production at its disposal, has control at the same time over the means of mental production." ${ }^{12}$ In Marxian terms, 'ideology' is what normalizes a certain political structure and power distribution as necessary and inevitable. To add to this, Althusser's Reading "Capital" reverse-engineers the mechanism of ideology by unpacking "the mirror myth of knowledge as the vision of a given object" (1970, p.19). He equates ideology with the production of knowledge, or rather, the production of "the knowledge effect of scientific knowledge", which is continuously reproduced as empirical reality by ideological state apparatuses (1970, p67). Ideology, then, is a distortion of reality that stands for reality itself, and to call its name is to expose it. The connotation of deceit is now reflected even in its dictionary definitions like the one of the Collins Dictionary of English which describes 'ideology' as a "speculation that is imaginary or visionary"13, and the Google definition that lists "visionary speculation, especially of an unrealistic or idealistic nature" ${ }^{14}$ as one of the

\footnotetext{
${ }^{12}$ Marx and Engels, The German Ideology quoted in Peter Brooker, A Concise Glossary of Cultural Theory

${ }^{13}$ Definition of "ideology" in Collins English Dictionary. Copyright $(\subset$ Harper Collins Publishers [Online] http://www.collinsdictionary.com/dictionary/english/ideology (Retrieved Nov 26, 2016)

${ }^{14}$ Google search for 'ideology' Nov 26, 2016

https://www.google.ca/search?q=ideology\&rlz=1C1TSCD_enCA463CA463\&oq=ideology\&aqs=chrome ..69i57.2680j0j1\&sourceid=chrome\&ie=UTF-8\#q=define: + ideology
} 
meanings. Therefore, to expose, or even suspect the presence of an ideology one needs a metalanguage of the kind Burke and Baudrillard provide.

Although similar in problematic, the two authors differ in orientation and devise their analytical tools in accordance with their goals. For Burke it is important to determine how language functions ideologically, whose, and what are the intentions that it mystifies. Therefore, his method assesses relationships and hierarchies constructed through speech, and attributes the ideological inclinations of language to the intentions and desires of a speaking subject. Baudrillard, on the other hand, is more interested in the specific social and cultural conceits that ensue from the ideological effects of representation. He, however, attributes these effects to seemingly opposite causes: it is the obsessive insistence on objectivity that disconnects the speaker from the means of representation, the sign from its content, and meaning from its reciprocity. (The "objective" sign born in this process is independent, an abstract entity of absolute truth value and validating impact.) Thus, while Burke constructs a critique of the absolutism of representation through a very thorough and deliberate examination of motive, Baudrillard keeps it at bay by means of "theory fiction" - a self-reflexive type of writing that avoids certainty and singularity of interpretation. As both their strategies facilitate the continuous renegotiation of meaning, Burke's choice of motive as the prism of analysis gives precedence to the subject, while Baudrillard's focus on the form of the sign as the premise of social analysis examines the subject in subordination to pre-existing communicative structures. Dressed in Burkean terms, Burke's own discourse can be loosely described as agent-centered, whereas Baudrillard's can be categorized as scenic (agent is influenced by circumstance). Therefore, as I apply their critical paradigms on the study of gamification rhetoric and 
discourse, I use the former to explore how the subject wishes forth and shapes its circumstances, and the latter - to reflect on the manner in which these circumstances then condition the subject.

In the following pages I describe in more detail Burke's dramatistic and rhetorical analysis and demonstrate how it can be applied to the examination of gamification rhetoric. Then, I review the tenets of Baudrillard's critical paradigm that facilitate the evaluation of the social and cultural implications of the gamification phenomenon.

\section{Kenneth Burke}

According to Kenneth Burke's philosophy humans are symbolic beings whose relationship to the world is enabled and determined by symbols. As he comments: "Symbolic communication is not a merely external instrument, but also intrinsic to men as agents. Its motivational properties characterize both "the human situation" and what men are "in themselves"" (Burke, 1969a, p.33). Thus, the symbolic systems we create and inhabit are representational, but also contain attitude and intention. They are a way of knowing the world, and a way of acting in it. By focusing on the dynamics of communication and the form of messages, in addition to their content, Burke establishes a standpoint for critical analysis which does not fall under the direct influence of its subject matter. Similarly, the examination of gamification as a rhetoric, rather than a mere statement, offers an insight into both outwardly spoken and implicit intentions of the gamification proposition. As a way of speaking it already is an action with an underlining purpose, and its consistent 'mannerisms' are more revealing than its claims. 
The resources of Burke's dramatistic and rhetorical analysis help locate the source of that action, but also outline the participants, their roles, goals and hierarchical relationships. The following pages give an outline of my method along with the Burkean terminology which facilitates it.

\section{Elements of the Pentad}

The first step towards a dramatistic analysis of a text is to determine the distribution of the dramatistic pentad or the roles ascribed to different participants in the action. In $A$ Grammar of Motives, Burke explains that the "titular word" he uses to describe his method is "dramatism", because "it invites one to consider the matter of motives in a perspective that, being developed from the analysis of drama, treats language and thought primarily as modes of action" (Burke, 1969a, xxii). He also observes that the "basic forms of thought" or patterns of expression which characterise drama, are common to all statements and attributions of motive. These Burke systematises in a "pentad of key terms" which signify the core relationships expressed in language:

In a rounded statement about motives, you must have some word that names the act (names what took place, in thought or deed), and another that names the scene (the background of the act, the situation in which it occurred); also, you must indicate what person or kind of person (agent) performed the act, what means or instruments he used (agency), and the purpose. (Burke, 1969a, xv)

Act, scene, agent, agency and purpose are the basic elements on which Burke's grammar of motives is build on. It may seem that the pentad is merely renaming the familiar parts of the sentence of conventional grammar: verb, subject, object, adverb, adjective. However, for Burke this change in terminology is needed to emphasise the fact that his 
grammar is concerned with the location of motive and force of action rather than the formal structure of language.

Drawing on Burke's understanding of speech as action, I will first read sample texts in order to determine: "what was done (act), when or where it was done (scene), who did it (agent), how he did it (agency), and why (purpose)" (1969a, p.xv). For example, this is how the approach can be applied to McGonigal's claim: "Games make us happy because they are hard work that we choose for ourselves"(2011, p.28). It appears here that 'games' are the agent since they perform the action of making us happy. To answer the question 'how they did it', one has to recognize that games make us happy by virtue of being equated with "hard work that we chose for ourselves". Rather than using any instruments, games themselves are also the instrument or the agency. Since the scene (the when and where of the act) is not specified, the statement should be considered valid under all circumstances, and is therefore a general truth. Finally, the purpose (the why of the act), which has also not been stated explicitly, must be assumed to coincide with the act itself ("make us happy"). The ambiguity here is further intensified by the fact that the purpose, or the original motive is hard to attribute to either "us" (the sufferer of the action) or games (agent/agency).

\section{Pentadic Ratios}

To name the act, agent, agency, scene and purpose of a claim is the first step towards the analysis of motive. According to the Burkean scholar Glenn Stillar "a pentadic analysis would be a mere cataloguing of the ideational structure (process, participants, and circumstances) of a text if it did not recognize the structuring capability of ratios" (1998, 
p.64). Burke describes the ratios, the particular combinations of elements in a given text, as "principles of selectivity rather than as thoroughly causal relationships" (1969a, p.18). Being a matter of an authorial decision, ratios help direct attention and construct motives, attitudes and orientations. For example, a scene:act ratio would treat the act as the natural consequence of given circumstances, or an agent:act ratio would suggest that the act was intended by the agent. For example, in McGonigal's statement ("Games make us happy because they are hard work we choose for ourselves.."), we observe a sequence of ratios which help situate the motive of the act. To begin with, the main clause constructs an agent:act ratio. The act (making us happy) is accomplished due to the capacity of the agent (games). On the level of the qualifier, however, the capacity or agency of "games" is based on their equation with "hard work that we choose". It appears that games as an agent/agency are determined by the co-agent "we" which is placed in the subordinate clause of the sentence. Hence, the ratio changes to co-agent:agency/agent:act. In other words, the initial sufferer of the act of games "us"/"we" qualifies the agency (hard work) that determines the agent (games), and ultimately owns the purpose of the act. In a single sentence McGonigal constructs a statement whose inbuilt ambiguity is expressed in a sequence of simultaneously existing and yet contradictory ratios of elements. There is a conspicuous doubling of the functions of the pentadic elements as games are both agent and agency, the player is both the sufferer of the act but also the main actor, and the act of making happy coincides with its own purpose. According to Burke, the possibility for multiple or layered interpretations embedded in texts is not a deficiency in clarity, but an expression of the "underlying enigma" of motives, as well as an important rhetorical device (1969a, xviii). Therefore, the purpose of dramatistic and rhetorical analysis, is to 
"study and clarify the resources of ambiguity" as the strategic moments of transformation (1969a, xix). What he is referring to are transformations in definitions, or changes in the "casuistry" from the perspective of which certain pentadic elements and ratios are made possible. As he says:

At every point where the field covered by any one of these [pentadic] terms overlaps upon the field covered by any other, there is an alchemic opportunity, whereby we can put one philosophy or doctrine of motivation into the alembic, make the appropriate passes, and take out another. (Burke, 1969a, .xix)

Thus, while the doubling of the pentad in McGonigal's sentence can be easily attributed to the structure of passive voice expressions, from the perspective of dramatism, the choice of the author is of greater interpretative significance. Why would McGonigal choose the passive voice over the active when constructing her sentence? Why is it important for her to emphasise that games can act, that games are hard work, over the fact that games are defined as such only by the person who plays? Similarly, why is the player construed as both acting and acted upon? This is a clear invitation for the renegotiation of definitions.

\section{Substance}

While the object of dramatistic analysis is the language of the text itself, the way in which a text foregrounds the pentadic elements pertains to an already established social framework and worldview. As Stillar explains, "every representation is the situated social practice of real social agents, who necessarily construct "reality" with reference to their practices and the terminologies that are a part of them" (1998, p.64). However truthful or straightforward these terminologies may attempt to be, they are necessarily synoptic and discriminatory. Burke points out that while we seek "faithful reflections of reality", we 
"develop vocabularies that are selections", and by extension, "deflection of reality" (1969a, p.59). The distribution of pentadic elements and ratios in a text are the result of these processes of selection, reflection and deflection. Moreover, ratios are not only responsible for directing attention and creating attitudes, but are conditioned by an existing system of definitions or terms, which Burke also calls "substantives". These are the in-text iterations of conventional beliefs which appear as references to the essences of things or reliable facts of life. However, according to Burke, the concept of "substance" itself contains and expresses the paradox of definition. Looking at its etymology, Burke notes that the word we use to describe what a thing "intrinsically is" refers to something on the outside of it - "something that stands beneath or supports the person or thing" (1969a, p.21-22). In other words, "the word "substance", used to designate what a thing is, derives from a word designating something that the thing is not" (Burke, 1969a, p.23). According to Burke, there are several types of definition that are responsible for the kinds of "substance" one can encounter, and each type expresses a different aspect of the "paradox of substance".

Contextual definition, for example, has to do with locating or placing the thing within its "natural" environment or scene. The word "define" itself suggests that to "determine a thing is to mark its boundaries" (Burke, 1969a, p.24). Based on the mode of defining, which assumes a formative relationship between a thing and its temporal or spatial context (scene) Burke arrives at the concept of geometric substance (1969a, p.24). The philosophical origin of this figure of thought that allows or rests on the reversal of "the intrinsic" into "the extrinsic" is traced back to Aristotle and Spinoza (1969a, p.2426). Burke observes how Spinoza's ideas on God and/or nature as the "total context, the 
universal scene" layered on the Aristotelian notion that the essence of each thing is to be found "in itself" forms the irresolvable ambiguity at the centre of contextual definitions. As he explains, "to see things as contextually "determined" by the "absolute" is thus to see them simultaneously in terms of "necessity" and "freedom"'" (Burke, 1969a, p.25-26).

Familial definition is another way of formulating "what is". Descriptions in terms of "ancestral cause" refer to "'general", "generic", "genetic", "genitive"' affiliations which serve to identify the members of a class, genus, tribe, family (Burke, 1969a, p.27). Although these too can be seen as definitions based on context, Burke points out that while "contextual definition stresses placement, ancestral definition stresses derivation" (1969a, p.28). When the essential constitution of something is construed by extension of its origin or derivation, Burke speaks of familial substance. He traces the beginning of this type of thinking back to Aristotle's idea of genus that was "originally not a logical, but a biological, concept", and Plato's doctrine of archetypes or perfect forms in heaven from which every worldly thing has been derived (Burke, 1969a, p.27-28). The presence of the "ancestral notion" is also discernible in the founding principles of Western feudalism and Nationalistic ideologies. This type of substantiation draws on the ambiguous relationship between the part and the whole, the individual and the generic quality as each defines the other.

If familial substance is rooted in derivation and possession, directional substance presupposes definition in terms of tendency and motion. Burke considers it "biologically derived from the experience of free motion" of the human body, and therefore emphasising "the sense of motivation from within" as pertaining to essences (1969a, p.31). As he points out, thought in terms of directional substance has gained prominence 
since the Renaissance and has been the main rationale behind doctrines of capital and techno-scientific progress. The implicit ambiguity at the center of this type of substance stems from the complexity of "motivation" which affords the transformation of intrinsically originating "free" movement into extrinsically "determined" motion and vice versa. In addition, defining with regards to "tendency" is defining, in equal measure, through a capacity that may or may not be expressed in the future, therefore is both present and absent.

Undoubtedly, for Burke all definitions or assertions of substance have one thing in common, and it has to do with the fact that "we necessarily define a thing in terms of something else" (1969a, p.33). The resulting ambiguity of substance or "agonism" of opposites is not only inevitable, but the core of any definition. In that sense, geometric, familial and directional substance can all be considered "special cases" of the more inclusive category of "dialectical substance" where one term of the dialectical pair constitutes the transcendence of the other.

Burke's treatment of substance as a paradoxical and agonistic entity reminds of the gap between representation and experience which we have to overlook for the sake of foundational "truths". In that sense, determining the presupposed substances within a text is to observe the function and circulation of the taken-for-granted beliefs of a given culture. To return to my example, the agent:act ratio in the main clause of McGonigal's claim is supported by the directional substance of games, which are defined in terms of their potential to make us happy. Their essential quality or 'true nature' is based on an inherent tendency. In other words, their way of acting/impacting is, in fact, what characterizes them best. It is due to that tendency or capacity to entertain that they are 
capable of completing the action. In the subordinate clause of the sentence, however, the ratio changes to agency:agent, and thus the 'substance' of games is determined by the "generic" qualities they share with "work" as another member of the same class of activities. Hence, games are also described in terms of familial substance. Finally, the coagent:agency/agent ratio reaffirms the familial substance of games, but adds an additional trait which exceeds the typical profile of its class. In contrast to work, games are also "chosen", and since they are "chosen" there is another agent that dominates the act. This brings us to the "original" or active form of the sentence, where games function as agency: "We play games to be happy". The question remains, what is the rhetorical intention of McGonigal who selected these particular ratios of the pentadic elements, and reimagined games in terms of directional substance?

\section{Rhetoric}

So far, Burke's dramatistic grammar relies on two important groups of terms to describe the construction of motive. The pentadic elements and ratios as Stillar explains, "highlight the relationships between terms of a text as the means for giving reasons", and the particular types of substance "furnish them with ostensibly inalienable kinds of "being"” (1998, p.72). Together, these lay the ground for the work of rhetoric which Burke has nick-named "stratagems with 'you' and 'me' quality about them" (1969b, xvii). The means of rhetoric can be synthesised in the following terms: addressivity, identification, transformation, and order. The 'you' and 'me' quality of rhetoric stratagems places addressivity in the centre of their definition. However, this is not to say that only rhetorical speech is addressed, but rather, that any speech is rhetorical, because 
it draws various participants into a common ground of understanding and action.

Moreover, the presence of language, according to Burke, both gives away and constitutes the alienation of human beings from each other, and from experience. Humans as "symbol-using" animals who are "separated from their natural condition by instruments of their own making" (1966, p.16), are also necessarily divided by different abilities, interests, beliefs, social status, class. Difference requires order, and order is created through "the mysteries of hierarchy", which permeate the fabric of "symbolic action". Hence, Stillar explains, "the function of rhetoric is to overcome division through identification and consubstantiality" (1998, p.73). To identify with another means to describe the world in overlapping terms or see it unified by similar substance. And yet, finding common ground in definition or interest does not mean becoming identical with another. As Burke writes:

In being identified with B, A is "substantially one" with a person other than himself. Yet at the same time he remains unique, an individual locus of motives. Thus he is both joined and separate, at once a distinct substance and consubstantial with another. (Burke, 1950/1969b, p.12)

By overcoming division, at least momentarily, identification initiates a transformation in the hierarchy. In other words, the purpose of the rhetorical act is the re-negotiation of the social order which produces it.

Looking again at McGonigal's claim ("Games make us happy because they are hard work we choose for ourselves.."), one can notice that even a single sentence can have rhetorical value. So far, the grammatical structure of the sentence rests on two opposing ratios that seem to cancel each other: the agent:act ratio where games act upon us, and the co-agent:agent ratio where we act upon games - our choice constitutes them as such. The first one defines games in terms of directional substance for their potential to 
make us happy. The second one, treats games in terms of familial substance by identifying it as a special category of work. The ambiguity of substance here indicates the awareness of the author of an existing division in opinions and attempt for renegotiation of definitions. Clearly, the popular definition of games presupposes a player who chooses to engage with an activity for its entertainment value or for its own sake. By structuring the claim the way she does, McGonigal attempts to convince us that games can be seen as consubstantial with work, and that their impact upon us is less dependent on our 'choice' than their inherent capacity to produce happiness. This amounts to the following transformations: games are re-defined as work and as a mysterious power, and the reader is invited to identify with that new definition. In fact, the use of words like "us" and "we" already assumes the consubstantiality of readers and writer.

\section{Logology}

As a final step of the analysis of sample texts, I also consider them as logological systems or as contributing to the discourse of gamification as a larger logological system.

According to Stillar, Burke's logology extends grammatical and rhetorical analysis "to the symbol systems themselves" (Stillar, 1998, p.77). Treated dramatistically, different discourses or philosophies exhibit motives and facilitate specific modes of action that are recognized and sustained by the presence of their respective ratios and substances. These discourses are, in their own right, "terministic screens" which seek faithful reflection, but select and deflect "the real". On this premise, I will treat gamification rhetoric as a comprehensive system of beliefs, expressed and asserted through a particular way of speaking, with its inherent attitude and program of action. This will allow me to examine 
the place and function assigned to the gamified subject as a technological agent, and consider the possible real-life implications for social actors implicated into a symbolic system of this kind.

As I demonstrate above, even a single sentence can offer abundant information about the author's motives and supporting conventions when subjected to dramatistic and rhetorical analysis. The application of the method to larger bodies of text offers more breadth, but also precision to the interpretation. Since it is impossible to analyse every sentence of every one of the chosen books, I focus on key passages such as the introduction, the conclusion, chapter titles, and definitions of game, happiness or fun, meaningful engagement, reality. I also seek out the places of transformation in pentadic ratios as the markers of rhetorical devices.

\section{Jean Baudrillard}

Jean Baudrillard is another theorist who founds his social critique on the analysis of signification processes rather than the "realities" they construct. He measures society by the nature of its symbolic action condensed in the form and structure of the sign. If Burkean Dramatistic grammar suggests that philosophical concepts should be treated as stratagems of the "you and me" kind where theoretical thinking is inevitably an exchange between interlocutors, Baudrillard treats our relationship to signs as indicative of our relationship to experience. Burke, who studies grammar as action and motive as worldview, still reads the history of philosophy through the perspective of an active subject. Baudrillard, who posits the structure of the sign at the premise of his analysis, emphasises the subject's pre-determination by existing communicative structures, and 
treats society as the concrete expression of its signification processes. As a theorist who would rather rely on the affective impact of representation than its fidelity to a confirmable truth, Baudrillard offers the right point of reference for the study of artefacts whose real value emerges as a by-product of the affect they produce. By definition, the products and applications of gamification, whose intention is to treat human emotions as a raw material for the production of cooperation, goods and profits, should fall in this category. Bellow, I trace briefly the evolution of some of the notions central to his critical paradigm and the discussion of the gamification phenomenon.

Baudrillard is a writer whose critical provocations win him great acclaim but also notoriety. He is commonly criticized for his sweeping generalizations, excessive irony, word games and self-contradicting claims. Indeed, too literal a reading of his highly abstract works will be misguided and prone to misconceptions. Baudrillard himself provocatively states that he respects reality far too much to believe in it $(2006, \mathrm{p} .4)$. His disposition is well aligned with his writing style, which is increasingly antagonistic and self-ironic in the later years of his career, when he devises a name to describe his own writing strategy. His "theory fiction", "simulation theory" or even "anticipatory theory" is meant to sabotage the conventions by reversing the traditional goals of theory - namely that of describing reality, producing results or predicting a future. Instead, he lays his bet on the seductive power of provocation and affect. In the foreword to The Intelligence of Evil, Chris Turner points out the "poetic (and often ironic) dynamic" of Baudrillard's language which "underwrites the authenticity of the symbolic order and its various forms" (2005, p.7). As Turner explains, "this spiraling play between concepts" and intentionally paradoxical terms aim to "preserve a secret, to guard a mystery" (2005, p.7). Dan Fleming 
and Damian Sturm, authors of Media, Masculinities and the Machine, also recognize that the value of Baudrillard's writing is not so much in the theoretical or analytical tools it offers, but in its ability to organize these "nonrepresentational stagings of affect (2011, p.7). The emotional impact of these stagings supersedes the particular representational details that lead to it, and leaves a lingering sense of an event, and a "feeling that we have grasped something" (2011, p.8). Thus, his body of work predominantly focused on the role of representation as the necessary go-between "the real and the imaginary", is carried out in somewhat of a prophetic tone, and takes the shape of a comprehensive diagnosis of the contemporary condition.

\section{The Link between Symbolic Action and Political Economy}

The theoretical genesis of Baudrillard's widely popular concept of the "simulacrum" as the simulation of a simulation without original referent begins far before the 1981 book of the same name: Simulacra and Simulation. In The Political Economy of the Sign he draws a parallel between the Saussurian, or Structuralist, division of the sign into signifier and content (signified + referent), and the Marxian explanation of the relationship between exchange value and use value. Baudrillard contends that it is this disconnect between sign and content, or lack of conscious investment and possibility for reciprocity in the signification process that generates political economy as its respective reality. In other words, the structure of the sign, or the kind of signification that is "the code", produces its corresponding social order. Although, Baudrillard does not strictly define "the code", neither its alleged alternative in "primitive" societies - "symbolic exchange", the concept emerges in the process of its use within his texts. As a thorough analysis of how and why 
Marxian critique fails to present an exit point or a radical alternative to political economy

The Mirror of Production is also a text which examines closely the workings of "the code". The code's alleged objectivity in combination with its detachment from any external referent facilitate a kind of logic that evolves and functions independently of any original cause while successfully maintaining its absolute claim on truth. Due to the functioning of this self-legitimating signification mechanism, the alternatives to the founding notions of political economy that Marx proposes prove to be their ideological justifications. One after the other, dialectical oppositions erected by Marx as radical critique of their counterparts in political economy become corrupted by the code which melts their apparent contrasts into sameness. Thus, exchange value and use value, concrete and abstract labour, work and non-work are shown by Baudrillard to rest upon the same fundamental definitions, namely that of man as labour power and history as production. Worse than that, the appearance of possible alternative that Marx creates with the notion of use value, only serves further the purposes of political economy. By providing it with an ideological horizon that is not radical enough to facilitate transcendence, it mirrors back the capitalist universe as the total real. For Baudrillard, this is all due to the holographic nature of signs and the code:

In fact the use value of labour power does not exist any more than the use value of products or the autonomy of the signified and the referent. The same fiction reigns in the three orders of production, consumption and signification. Exchange value is what makes the use value of products appear as its anthropological horizon. The exchange value of labour power is what makes its use value, the concrete origin and end of the act of labour, appear as its "generic" alibi. This is the logic of signifiers which produce the "evidence" of the "reality" of the signified and the referent. (Baudrillard, 1975, p.30) 
According to the logic of the code, the name proves the existence of the thing it names, hence the name takes precedence over the thing it names, to the effect of the code's complete independence from any original referent, but also the obsoleteness of the original referent. Thus, Baudrillard uses Marx' critique of political economy to demonstrate that the moment concepts take on "universal" meaning or become "a principle of explication" (1975, p.47), rather than interpretation, they cease to be subversive and cross over into the sphere of "repressive simulation" (1975, p.48). From then on, the meaning and validity of signs are exclusively secured on the basis of their relationship with neighbouring signs, hence leaving the authority of the code unchecked: And although at the best of times these concepts have been practiced as concepts without taking themselves for reality, they have nonetheless subsequently fallen into the imaginary of the sign, or the sphere of truth. From this point on they only invoke themselves in an indefinite metonymic process which goes like this: man is historical; history is dialectical; the dialectic is the process of (material) production; production is the very movement of human existence; history is the history of modes of production, etc. This scientific and universalistic discourse (code) immediately becomes imperialistic. ( Baudrillard, 1975, pp.47-48)

It is notable how Baudrillard's deconstruction of taken-for-granted truths seems to go through stages that are well in agreement with a number of Burke's notions describing different modes and principles of representation. Baudrillard's discussion of productivity and labour power subverts their status as fundamental notions by virtue of illuminating the "paradox of substance" that is inherent to their definitions (ex: defining use value in relation to exchange value), the "ambiguities" that arise in the process, and the "transformations" that occur for one dialectical opposite to be reduced to its respective other. In doing this, Baudrillard is also in agreement with Burke's claim that representation is necessarily selective, and although the underlining aspiration is for an 
accurate "reflection of reality", what occurs more often than not is some kind of "deflection". What Baudrillard calls "indefinite metonymic process" can also be read by Burke as the use of the familial substantive that brings out equivalences in notions that eventually leads to the elimination of resulting "tautologies". Following the logic of this progression, which Burke describes as the narrowing of the circumference of a notion's treatment, both authors illuminate the evolution of concepts or the arbitrary emergence of "universal" truths. An example that appears in both is the evolving meaning of the concept of nature. Burke explains how by reduction of the "concept of reason in God's order", to its narrower interpretation as "natural law", then "materialism", then to the concept of "environment" and even more specifically, the type of environment determined by "economic factors", we finally arrive at the very circumstantial interpretation of the economic as restricted to "the monetary and financial" as natural laws (Burke, 1969a, p.91). In a similar fashion, Baudrillard describes how the concept of nature, which in the 17th century is associated with "the totality of laws founding the world's intelligibility" namely God, after the Enlightenment is reduced to "Nature as a potentiality of powers; the primordial source of life" and thus enters "the era of its technical domination", where it is read as the "sign of the principle of production" (Baudrillard, 1975, p.54). By the logic of this gradual reduction of circumference, or the rationality of the code, Nature and man are finally equated with "economic factors of production" (Baudrillard, 1975, p.55). Thus, whether they call it 'narrowing of circumference' or 'metonymic process', both authors observe the signification process by which the notion of God as the reason of all things, is equated and then reduced to nature as matter in motion, and then to economic factors and the principle of production. In other words, the effects of the code seem to coincide with 
those of rhetorical definition where dualisms born out of the paradox of substance progress toward consubstantiality of opposing notions that prepares the elimination of one of the extremes. Moreover, the code functions according to the principles of signification described by Burke, because it is a rhetoric which forgets its original speaker and assumes an air of generality. Its reality effect, continuously intensified by its increasing detachment from original referents of any kind, is what Baudrillard persistently cautions against in his subsequent works.

\section{Stages of Simulation}

One of his latest books, The Intelligence of Evil offers an updated discussion on the function of the code in a virtualized environment. Here, once again, he rehearses the idea that "the invention of Reality, unknown to other cultures, is the work of modern western Reason” (Baudrillard, 2005, p.39). 'Primitive' or symbolic societies, according to Baudrillard as quoted by Turner, "still knew how to handle [the world of radical] illusion" (2005, p.12). Their make-believe world was both immediate and reverent of the inevitable distance between lived reality and its abstract sublimations. 'Objectivity' as this perpetual "concretizing, verifying, objectivizing, demonstrating" becomes a necessity only after "one is no longer sure in the existence of God, or when one has lost the naive faith in a self-evident reality" (Baudrillard, 2005, p.39, p.19). If the invention of objective reality is already a representation, the following stage naturally is that of the "simulacrum" as the representation of representation or the copying of a copy, which conceals the absence of the real or the original. However, in The Intelligence of Evil, Baudrillard is no longer satisfied with the exhaustiveness of 'simulacrum'. If his former description of the society of spectacle emphasized that "reality has succumbed to artifice", here Baudrillard finds it 
more accurate to state that after the loss of the sign, of images, and artifice, we "are left with absolute reality" (2005, p.67). He writes:

What we have in virtuality is no longer a hinterworld: the substitution of the world is total; this is the identical doubling of the world, its perfect mirroring, and the matter is settled by the pure and simple annihilation of symbolic substance. (...) We have moved, then, from objective reality to a later stage, a kind of ultra-reality that puts an end to both reality and illusion (Baudrillard, 2005, p.27)

As it seems, the natural evolution of "objective truth/reality" is to proceed towards its other extreme - that of complete relativity where all simulations are equally present and equally valid. The perfect models it produces are frozen in their own repetition as exact replicas of themselves, diverging ad infinitum from the real as becoming.

Baudrillard's claims may sound extreme if one is to forget that they constitute a commentary on our treatment of signs, symbols and artefacts. He himself continuously reminds the reader that the treats he sees regard the realm of concepts first:

Let us be clear about this: when we say reality has disappeared, the point is not that it has disappeared physically, but that it has disappeared metaphysically. Reality continues to exist; it is its principle that is dead. (Baudrillard, 2005, p.18)

However, the observed changes in our metaphysical understanding of the world are bound to affect our ways of being too. This contemporary way of being is what Baudrillard calls "Integral Reality". Unlike the "simulacrum" which is the basic principle of confusion of the sign with its content, Integral Reality is the consequence of that confusion. In The Intelligence of Evil Baudrillard goes to great lengths to describe its impact on everyday life. Some of the changes he points out include the demand of digital technologies for "heightened participation" (2005, p.31); the obsoleteness of the notion of time brought about by constant immersion in "immediate connectivity" (2005, p.30); and the confusion of experience with its shared 'real time' representations. As such, integral reality is also 
characterised by its artefacts which take after its form and content and reproduce it. Their means of expression and structure blur the distinctions between real and imaginary, virtual and lived experience, present and future, subjective and collective perspective, man and his digital traces, or man and machine. Baudrillard describes the impact of the code and its products in somewhat poetic terms:

What was once separated is everywhere merged. Distance is everywhere abolished: (...) between the subject and the object, between the real and its double. And this confusion of terms, this collision of poles, means that nowhere is value judgement now possible anywhere any longer: (...) By the abolition of distance, of the 'pathos' of distance, everything becomes undecidable. (Baudrillard, 2005, p.75)

The elimination of distinction and judgement, "this collision of poles" Baudrillard speaks about is the collapse of the familiar strategies of understanding based until recently on dialectical thinking, and expressed in the Burkean "paradox of substance". To consider a thing in relation to another allows space for discussion of difference, for the negotiation of meanings, for the awareness that every definition is an interpretation in transition. On the contrary, validation or verification on the basis of infinite equivalence achieves its absolute confidence in a real untainted by subjective interpretation - a real as indifferent and nihilistic as it is absolute. In the absence of the subject and motive, integral reality does not distinguish between fact and artefact. (If there's no subjectivity, there is no meaning, because meaning is subjective.) The collision of fact and artefact is the central stated goal of gamification which seeks to produce real-life results through immersion in fun, virtual environments. By definition, the gamified experience is designed with the intention of predicting and controlling future interactions and their outcomes. Available courses of action amount to a number of possible scenarios all of which have a pre- 
assigned meaning. In that sense, the future coincides with the present, and the gamified subject coincides with his/her electronic trace.

It is safe to suggest that, while Burke and Baudrillard start at different departure points and use different methods of analysis, they have similar understanding on the effects of signification processes on social practice, the mechanisms of emergence of conventional truths, and even the content of these conventional truths as shapers of the contemporary condition. Burke's study of signification places the "paradox of substance" at the center of all defining. He sees it as the ironic but unavoidable slippage that happens at the core of human cognitive processes which tend to organise the world by means of analogy, association, and deduction or simply by finding similarities and contrasts between new and familiar things. Problems arise when we forget that every definition is only an approximation of the truth, and an interested one as well. To this Burke finds a resolution by devising a method of analysis which treats any text as a dramatic action, and the subject/speaker as an actor who distributes the roles. Baudrillard, on the other hand, speaks of the structure of signs, and the logic of representation as something not based in cognitive processes, but the result of the accumulation of arbitrary interpretations of representations that harden into philosophical conventions. Thus, "the code" - cold and rational, is historically defined by the philosophies of the Enlightenment as its point of origin, but it continues to evolve and shape our world today. In a sense, Baudrillard's critique of the absolute claims of the code, and lament for the impossibility of "symbolic exchange" is precisely a call for recognition of the role of the subject as a speaker and organiser of reality. Similar to Burke who analyses for and through rhetorical intention, 
Baudrillard believes that there should be a possibility for "conscious investment" in signification, as well as reciprocity in the making and exchange of signs. 


\section{CHAPTER TWO}

\section{The Grammar and Rhetoric of Gamification}

The ideological functions of language come to prominence in rhetorical speaking to such a degree that rhetoric is understood only as the art of persuasion. For this reason, rhetoricians often feel obliged to differentiate the discipline from its object of study, or else - the science of rhetorics, from rhetorical means of communication. With the caveat that rhetoric is not only the art of persuasion, but also the critical framework for its examination, I use the Burkean analytical paradigm to disentangle the persuasive means of gamification rhetoric. Based on the relations of power and motive constructed by the analysed texts, I claim that gamification advocates are rhetors who coach an audience of business leaders or prospective gamifiers how to transform games and game-players into instruments of efficiency and productivity. The examined books establish an hierarchical order, according to which the gamified subject is grammatically overpowered by the game as well as the gamifier.

The chapter takes several books on gamification as examples of gamification

promotion rhetoric. These artefacts, or samples of rhetorical speech, are selected from a much larger body of literature on the application of game strategies in everyday life. I 
have divided gamification advocates in two groups. In the first one are authors who write what can be described as textbooks for the utilisation of games to pragmatic or business ends, while the second group is composed of writers who see a lot of humanistic promise in the democratic nature of games. The assignment of different authors to either the 'humanistic' or the 'utilitarian' group is done based on their own statements of purpose. Roughly speaking, the group of authors I call 'humanists' tends to emphasise the benefits of gamification for the player or end user. They also treat games as a "common language" that is accessible for players and designers alike, and can advance the dialogue or coordination of goals and ideas in an array of non-game contexts. On the other hand, those I describe as 'utilitarian', tend to focus on the capacity of gamification to serve the larger goals of the business, the corporate owner, or any other organizational body. Like any classification, this one also relies on generalisation, but it offers a starting point for the analysis and it helps determine to what extend authors' claimed goals correspond to their actual dramatistic treatment of games, gamification, and the gamified subject. For representative samples of utilitarian gamification rhetoric I use the books of Michel Hugos Enterprise Games and Adam Penenberg Play at Work; and for representative sample of humanitarian gamification rhetoric I use Jane McGonigal's book Reality is Broken.

As a kind of counterpoint to the rhetoric of gamification promoters, I also discuss the book of Ian Bogost How to Do Things with Games as an example of gamification theory, or rhetoric on gamification. He is known as one of the first fervent critics of gamification as the exploitation of games and playfulness. However, the means of dramatistic analysis reveal the same hierarchical order even in Ian Bogost's theoretical 
discussion of games as a medium. Although, he defines video games more broadly as a new communication medium, his book still explores their instrumental function and persuasive value in various real life contexts.

The analysis of the sample texts proceeds roughly through the following steps: 1) determining the distribution of pentadic elements; 2) establishing the dominant ratios and transformations of ratios through the text; 3 ) observing the function of substance as ground for motive; 4) examining the use of the rhetorical means of identification and consubstantiation within an implied hierarchy.

\section{Utilitarian Gamifiers}

By its very structure, the word 'gamification' suggests the presence of at least two distinct entities: a game and not a game. Again, based on the meaning of its suffixes 'gamification' is a noun which has been derived from the verb 'gamify' (to make something game-like) hence it implies an action led by an intention. According to the authors I have grouped under the title utilitarian gamifiers, the opposition foregrounded by the notion is between games and the 'real' world of work and business; whereas the intention of the gamifying action is the utilization of games for business enterprises. To fit the purpose, their texts are usually structured as textbooks or guide books offering expert advice on most current labour organization practices, and are openly addressed to a corporate or entrepreneurial audience. The tone of voice is authoritative and reassuring, and the line of argument follows a common trajectory. It usually starts by emphasising the established opposition between work and play, as well as outlining the problem of insufficient motivation at the workplace. In contrast to tedious work, games are mysteriously attractive to everybody 
(average age of gamers 37, both women and men play), and statistics are quoted to show that great numbers of people willingly spend innumerate hours playing. The central claim is that the proven enthusiasm for video games can be harnessed to produce real-life benefits for both employers and employees. All of this is possible, because gaming is not that different from hard work. Most authors support this claim by direct or indirect references to Csikszentmihalyi's theory of optimal experience and Maslow's hierarchy of needs. Gamification becomes the recommended tactic for closing the gap between work and play, tediousness and motivation. To prove its efficiency, the more recent books offer substantial amount of examples for its successful application in a variety of business fields and work environments. In the following pages, I will examine closely the rhetorical devices employed by Michael Hugos in Enterprise Games, and Penenberg in Play at Work. The first teaches how to "gamify" work or how to "businify" games, and the second explains why pre-designed reality is more real and more functional than reality. The examination of these books allows a glimpse into what can be seen as the emerging ontology of utilitarian gamifiers, its grammar and its definitions of concepts like games, reality (present and future), human happiness and freedom.

\section{Hugos: Enterprise Games}

The framework of actions, actors, and motives in Hugos' book is multifaceted and requires examination in stages. The top level of interaction is defined by the relationship between writer and reader, and their common ground or scene of existence. This common ground is established by the writer who initiates the communication exchange, and is the carrier of the original motive. Next in importance is the relationship between games and 
gamifiers, as well as games and users immersed into gamified environments. These secondary level acts are best observed in the definitions of games the author provides. The hierarchical structure that incorporates all of the above acts and participants emerges as a system of action where gamifiers determine games, and games determine players. The tracing of the elaborate transformations in pentadic ratios provides the foundation for the further analysis of the persuasive devices and intentions of the author.

The preface to Enterprise Games draws out the basic parameters of the casuistry Hugos works within and legitimates certain possibilities for symbolic action. As a statement of motive, it readily identifies the relevant context, the speaker, the audience, and the intent of the message. Here the grammar of interaction is already set in place and the elements of the pentad and the ratios they form can be seen as an exemplar of the prevailing attitude and dramatistic structure of the rest of the book. Since the book is about a new approach to business, it begins by establishing the need for this change. Hugos makes this call from the position of an expert, who addresses an audience comprised of "change-minded business executives", "people who advise them", "people who design games", and those whose "work is already taking on a game-like quality" (Hugos, 2012, viii). This varied crowd of prospective readers are all united under the pronoun "we" as the residents of the contemporary condition:

We are living in a time of big changes. We face changes driven by powerful forces like world population growth; rising prices for food, fuel, and raw materials; depletion of natural resources...

(...) the conflict between wage payers and wage earners is returning. (...) Companies hire and fire as needed to respond to market volatility and (...) fit the realities of our real-time, global economy. (Hugos, 2012, vii-viii) 
With this description, Hugos draws attention to the common ground of existence, where writer and readers meet in a particular relation, but also establish the 'scene' as the counterpart of game - the 'real' or the not-game that has to be gamified. The variety of issues he lists here amount to the main problem posed by his book - economic hardships leading to business instability. By limiting the scope of the discussion to the exclusive perspective of business enterprise, Hugos cuts out a piece of 'reality', and uses it to signify the whole. Thus, the definition of 'the real' is reduced to the economic reality we inhabit. This procedure that frequently partakes in substantiation, Burke calls "narrowing of the circumference" of terminology. Burke clarifies that while every terminology is a selection of reality which to function properly needs scope, a selection becomes a "deflection when the given terminology, or calculus, is not suited to the subject matter which it is designed to calculate" (Burke, 1969a, p.59). In that sense, Hugo's reduction of reality to its economic aspect constitutes a deflection. This narrow definition of the world, however, furnishes the scene:agent ratio that dominates the passage. The implication is that 'we' the readers and author alike, are conditioned by our common circumstance.

The remedy Hugos proposes against the challenges of this economically troubled world are games. Since the currents of the new time cannot be stalled or reversed, he recommends the adoption of video game technologies that are immensely popular and present a promising model for structuring of complex multi-participant interactions. The author's treatment of games in the following passage throws light on the relationships between games and gamified subjects, as well as games and gamifiers:

Games and associated technology we currently refer to as video games offer us more than just diversion and escape from difficult times. They offer us field-tested models to use for organizing companies and performing complex and creative tasks. They offer clear and 
compelling examples for how people can work together, build their carriers, and earn a living in rapidly changing and unpredictable environments. (Hugos, 2012, viii)

It is interesting to notice the ambiguous pentadic function of games which are treated both as an agent and an agency. By saying that games "offer us field-tested models to use for organizing companies", the author assigns them a human-like capacity to act (games offer models). At the same time, 'games' are the agency or tool employed by "us" for the organisation of companies. Thus, in this sequence of related actions, games lead the first action forming an agent:act ratio, but are then demoted to a means towards the end of "organizing" in the second. If this second action is properly paraphrased in an active voice it will again be expressed by the agent:act ratio, however the agent will be "We" (formerly 'us'), and the act will be "use games as models for organising companies". However, this active form of the statement can be seen as the chosen destination of the author's message. It is what the audience can be convinced in, but only after following the transformations of the pentadic ratios which advance from scene:agent to agency:agent and finally arrive to agent:act. In other words, the agent who is initially conditioned by one's environment, proceeds to adopting a new strategy (applying games in business) that now brings him into the category of an expert entrepreneur. As such, the agent is capable of determining the action.

The author's desire to guide his readership towards the empowered position of the expert gamifier is also confirmed by his direct statement of intent. Here, however, the composite agent "we" falls apart into segments with various pentadic roles:

My intent is to arm and inspire those who are in a position to influence or change the way businesses and organizations operate. I draw on my own experience as well as on the writings and the experiences of others in business and game design in order to present real-world examples of the merging of games and business. These examples outline 
salient features of an operating model for companies and economies that can deliver broad-based and sustainable prosperity. (Hugos, 2012, ix)

This statement reveals that Hugos invites a specific group of people to identify with the idea that games can become work. These are the people "who are in a position to influence or change" business processes. The so far unifying "we" and "us" is suddenly narrowed to the exclusion of people "whose work is already taking a game-like quality". Thus the union between the business leaders that Hugos properly addresses, and employees or players that he excludes, is now made conditional on "the merging of games and business" that will "deliver sustainable prosperity". More importantly, the split of the agent "we" correlates with the ambiguous pentadic function of games noted in the previous passage. Both of these grammatical gestures construct the hierarchical order of participants in the interaction. By now, these participants constitute at least three different types of actors where business owners, individual employees, and games can be considered the respective representatives of each group. The dramatistic functions of these actors vary from one context to the next in correspondence with the specific relationship the author seeks to emphasise at any given moment. Hence, the ambiguous pentadic function of games may suggest that from the perspective of business owners games function as means to an end, i.e. agency. From the perspective of the end user, player, or employee, games appear as another agent, or scene.

The special quality of games that allows them to alternate between the functions of agent and agency is examined further in the more elaborate definition Hugos offers in Chapter 1. He writes:

A game is an engagement engine - it attracts and engages players. You can measure the success of a game by the number of players it attracts and the level of engagement it gets from its players. Games are specifically designed to attract and engage people through the 
application of the four traits introduced in Chapter I: goals, rules, feedback systems, and voluntary participation. Looking at these four traits you could say that the combination of the first three traits is what creates the fourth trait. (Hugos, 2012, p.12)

Hugos' treatment of games as actors is emphatic and consistent. It is games that "attract", "engage", "induce", "offer", and in every way act upon rather than bear the action of players. The condition to exhibit the "right combination" of goals, rules, and feedback systems that reliably "induce voluntary participation" is a qualifying one. If it were not met, a game cannot be qualified as a game. Here, games are defined in terms of directional substance, or inherent potential. The player, on the other hand who has to be "attracted and engaged" and is expected to exhibit "voluntary participation", appears entirely passive. Paradoxically, his intrinsic motivation is effected by external forces. Pinpointed as the locus of action games in this context can assume the function of either an 'agent' or a 'scene'. If games are understood as a 'scene' which constrains the player in a scene:agent ratio, they constitute an environment of impersonal forces driven by "physical causes rather than spiritual ones" (Burke, 1969a, p.131). This delineates a materialistic, pragmatist casuistry, from the perspective of which empirical facts are the result of material processes. By extension, humans too are little more than the mechanical outcome of matter in motion. However, a scenic interpretation does not eliminate completely the possibility for action. A "scenically" derived agent, as Burke explains, is an individual entity, a "finite or determinate mode of Substance", whose primary tendency is to "forever persist in its nature" (Burke, 1969a, p.144). Thus, by ascribing the role of agent to games, Hugos inadvertently claims that in addition to being a straightforward, mechanical process, a game is also a self-originating force, whose "being or essence is intrinsic to it" (Burke, 1969a, p.144). Therefore, its mysterious power or impact on the players as co- 
agents cannot be easily influenced from without or reproduced. The presumption being that once an interaction or a process turns into a game, it acquires a "will of its own", and becomes an agent.

The fact that we can differentiate between three different types of actors, and 'games' are alternatively attributed the pentadic functions of 'agent', 'scene', and 'agency', is an indication that Hugo's statement of motive unfolds on several planes of action. For the sake of simplicity, the top layer can be named Act1, and is best summarized by the statement: entrepreneurs use games to overcome economic hardships. By extension, scene 1 is the challenging economic environment, agent1 are the entrepreneurs, and games are the chosen agency 1 . The dominant ratio describing this claim is agent:act, i.e. entrepreneurs are in control of their own actions by means of games. On the next plane, Act 2 can be described with the claim: Games are feedback systems that engage and attract players. Here, games constitute scene2 (or agent 2), and they determine players (coagents) in a scene:agent (or agent:co-agent) ratio. Finally, the mention of players' "voluntary participation" is to suggest that players too should, at some point, become the locus of motive on a hypothetical plane of action that I will designate as Act3.

This multilayered structure of motive is detailed further in the numerous examples of successful business practices Hugos describes. One of them outlines the case of Mike Chakos early on in the text to demonstrate the positive impact of purportedly gamemodeled organizations. Hugos introduces him as a "serial entrepreneur" who "has successfully started and turned around five companies over the last two decades" (2012, p.8). His success is attributed to the fact that Mike "creates companies that directly appeal to the four essential human cravings and that also incorporate the four game traits into the 
way they operate" (Hugos, 2012, p.9). The essential human cravings as per McGonigal ${ }^{15}$ (satisfying work, hope of success, social connection, being part of something larger than ourselves) are addressed in a statement of Mike's business philosophy, which reads: "We share the opportunity with qualified people to do the job they like to do; have fun working; be part of something bigger than themselves; earn as much money as they are capable of earning; and live the entrepreneurial experience" (Hugos, 2012, p.9). The results of Mike's vision and business practice which also incorporates the four game traits (goals, rules, feedback systems, voluntary participation) are then summarized thus:

Mike gets enthusiastic participation from the people who work in his companies because he shares the opportunity to be a successful entrepreneur with a larger group than is the norm in most companies today.

The bonus plan has evolved to promote team success instead of individual success. (...) This creates an environment of sharing and helping, as well as a good dose of peer group pressure that keeps people focused on doing their best.

This bonus program is the vehicle Mike uses to set up and perpetuate a feedback system that engages company employees. It keeps them focused month after month and year after year on operating the company efficiently and doing what they need to do to achieve company performance and sales objectives. (Hugos, 2012, pp. 9-10)

Mike's statement of business philosophy offers a glimpse of the plane of action I have referred to as Act3. Here for the first time the players/employees are described in active terms as they "do the job they like to do", "have fun", "live the entrepreneurial experience". However, even these "free" activities, where employees momentarily appear as agents in control of their act, are framed by the actions of more dominant actors, who create and "share the opportunity", "get enthusiastic participation from the people" and "keep them focused". This contradictory treatment of employees as both the originator of

\footnotetext{
${ }^{15}$ McGonigal, J. (2009) Reality is Broken. p.49 quoted in Hugos, M. (2012) Enterprise Games. p.5
} 
the action, and means towards an end (agent and agency) is eventually resolved as the collection of quotes above gradually display the proper place of all participants in the hierarchy of motives. Mike (agent1) uses the game-like feedback system (agency1) to ensure the engagement of employees (purpose1). Feedback systems or games (scene2) engage employees (agent2) and keep them focused. Engaged and enthusiastic employees (agent3) do "what they need to do to achieve company performance and sales objectives"(purpose3). In isolation, each layer is an act in itself with straightforward pentadic distributions. However, when considered together, the three planes of action form a complex structure, where the function of elements shift to accommodate the integrated meaning. This integrated meaning is carried by the introductory sentence with which Hugos invites the audience to examine Mike's business practices:

In particular, let's take a look at one of the techniques Mike uses to get people's enthusiastic engagement in doing what it takes to make the business successful. (Hugos, 2012, p.8)

This one sentence unites and directs the various acts on multiple levels toward the same purpose - business success. Clearly, by virtue of the fact that Hugos writes an instruction manual on gamification, his whole narrative is dominated by the purpose:act ratio. "We" the readers and students of gamification observe its efficiency in the field. The study of gamification is justified by the successful business it promotes, i.e. the purpose motivates the action. On the next level, Mike - the object of observation uses techniques to get people's enthusiasm. Here Mike is the agent, his techniques are the means to stimulate people, and people's engagement is his purpose, which, in turn, becomes the means toward the end of business success. Mike is described as an expert businessman, because he has selected the right tool (agency) to serve the right purpose. In that sense we can say 
that the dominant ratio here is agent:act - an expert agent controls the act. Finally, at level 3 the agent is most complex. "People" as agent are determined by Mike's "techniques", which "induce" their enthusiasm and voluntary participation. Mike's techniques function as a scene, in which Mike's direct presence dissipates, but his agency remains in one with his purpose. Paradoxically, while people (agent) are determined by Mike's gamified business practices (scene), they are still presented, and must conceive of themselves as self-motivated, originators of the act of "doing what it takes to make the business successful". Thus we observe how, on the one hand, "people's enthusiasm" functions grammatically as Mike's purpose and then Mike's agency, and on the other, it is meant to signify and be interpreted as the presence of people's own intent. However, their subordinate pentadic function suggests that the motive originates elsewhere. By being subordinated to Mike's intention, and determined by Mike's techniques, 'people' in turn become agency and their behaviour is automated motion rather than conscious action. Once again, the locus of motive is found in Agent1 - the corporate owner Mike, who clearly initiates and directs the action on all three planes. Along with the gamified bonus program which acts to engage employees, employees themselves, their happiness, as in "fun", and "free will", as in choice of work, have become vehicles for Mike's successful business enterprise.

The varied landscape of pentadic ratios within Hugos' text serve his rhetorical intentions. The text begins by establishing a common ground between the writer and readers through the process of identification. The writer who assumes the position of an expert, is willing to share his expertise with perhaps less informed, but equally forwardthinking and "change-minded business executives" who are faced with the same 
challenging economic environment and share the same passion for success (Hugos, 2012, viii). The reader is thus called upon to identify with a specific social position, value system, and circumstance. On this basis, the initial distinction between the expert and the laymen can be overcome by following the prescriptions of the guidebook and adopting the recommended strategies. This is what the changing pentadic ratios in the introduction teach. Before being familiar with gamification (agency) the agent is determined by his environment (scene). After adopting gamification as means, he can initiate the act and manage the environment (agent:act).

The ratio transformations in the definition of games is also significant. As Burke points out, it is a familiar "resource of Rhetoric" to "deflect attention" by stressing one ratio versus another (Burke, 1969a, p.17). The emphasis on the active role of games in determining the behaviour of players, distracts from the fact that the true locus of motive is in the leading agent - the business leader, for whom both games and players are simply means to an end, i.e. agency. As per Hugos' example, the goals and interest of Mike's business enterprise are disguised behind an impersonal force, whose indiscriminate effects are democratic enough to foster the free volition and action of employees. In other words, games allow Mike to make his goal - profitable business, appear as people's own original intent. He persuades the employees, not by straightforward arguments, but by designing a scene which feels open and unpredictable enough for the workers to be convinced in the spontaneity of their 'voluntary' action. Simultaneously, Mike persuades the readers or entrepreneurs by demonstrating that games are a perfectly controllable environment that can guarantee the desired business results. Thus, the relationship between gamifiers and the gamified subject that Hugos promotes is one of unequal power 
distribution due to deliberately asymmetrical access to information. As far as the employee or game-player is only partially aware of the intended consequences of the gamified act, she is not its originator. According to Burkean Dramatism, "the basic unit of action would be defined as the human body in conscious or purposive motion" (Burke, 1969a, p.14). Based on this definition, the voluntary participation of the gamified subject, is an action that has been reduced to motion. Similar to the reader of the book who can redeem himself to a higher position in the established hierarchy by following its advice, the gamified subject finds his capacity for action preconditioned by his participation in the game. Hence, the text creates a double bind according to which the transformation of the proposed hierarchy is possible only by accepting and conforming to its demands.

\section{Penenberg: Play at Work}

With his book Play at Work: How Games Inspire Breakthrough Thinking, Adam Penenberg is another author who fits into the category of utilitarian gamifiers. As one of the more recent sources on gamification, the book takes the opportunity to consider and argue against prominent critics, as well as present an updated list of success stories of gamification applications. However, the overall intent of the message and structure of the argument follow a similar trajectory to that of Hugos'. Once again gamification is presented as a progressive solution to a variety of issues such as, "endemic unhappiness in the workplace", "three hundred billion dollars in lost productivity", "abject negativity" and deteriorating public health since employees who are "emotionally disconnected from their work [are] about as likely as the unemployed to report suffering from chronic illnesses, diabetes, and high blood pressure and cholesterol" (Penenberg, 2013, pp.11-12). 
Like Hugos, Penenberg proposes that "games and game mechanics can be, if designed intelligently, a powerful way to drive engagement", but he doesn't see their use and impact limited to the context of business enterprise (Penenberg, 2013, p.6). Although he offers multiple examples of the fact that "games are good for business", he also stresses that games are ubiquitous, "creeping into almost every facet of our lives", forming a "game layer" over the world (Penenberg, 2013, pp. 6-9). Not only do they form the cultural indoctrination platform for the young generation, but are an inevitable part of the organization of lifestyles in the future. To defend such large claims Penenberg draws evidence from various sectors where games and "gamelike elements" have already become part of routine activities and work procedures. These are corporations, the military sector, healthcare, marketing, education, scientific research, governance.

While the rhetorical intentions of this text remain largely the same as those of Hugos, what makes it interesting is its greater attention to the psychological and biological processes which comprise the scientific ground of the gamification proposition. Inadvertently, Penenberg offers insight into the conceptualisation and treatment of human emotion by gamifiers, as well as its placement in the familiar structure of hierarchical relationships. In addition, his vision of the role of "gameful design" for the future of humanity is unabashedly enthusiastic, giving his rhetoric a utopic hue. The gravity of Penenberg's claims does not stem from their loyalty to fact or premonition of value, but from the demonstrated intention for and orientation towards a specific reality and a future. The implications of a "hackable" human brain immersed in ubiquitous simulation emerge in the author's treatment of games, happiness and the real. 
Penenberg's treatment of games exhibits the distribution of pentadic elements already observed in Hugos' text. It is a distribution that preserves the familiar hierarchical structure, according to which the gamified subject is an actor at the lowest level of action who has been determined by the actions of all other actors before him. The following are two passages where the sequence of actions and the relationships between actors are outlined:

Play at Work is not about games per se. It is about harnessing the characteristics that make them so engaging and applying them to other aspects of our lives. These game mechanics can be applied to the workplace to make employees happier, more productive and motivated, to increase company profits and improve worker safety, to market new products, and to help with customer service. They can help people learn and better retain information, to create new products and solve big problems. And they can foster healthier lives. Because they are predicated on providing a system of principles, mechanisms, and rules that govern a system of rewards that lead to a set of predictable outcomes, they can ratchet up a person's engagement, and increase happiness and productivity, which in turn can pay big dividends. (Penenberg, 2013, p.11)

It (the book) illustrates how businesses from the smallest start-ups to non-profit organisations to schools to government agencies and the biggest multinational corporations are unleashing gameful design - the characteristics that make games fun and addictive - to increase worker productivity and job satisfaction, train employees, get them to communicate better and interact more, incent them to be more environmentally conscious, and contribute to the creative process. (Penenberg, 2013, p.12)

Penenberg's statement of intent reveals that his book is not about games, but about the possibility of utilization of games, i.e. their transformation into agency. Games are divided into essential components, then reduced to mechanisms that can be figured out, and reapplied elsewhere to produce the chosen results. This is a definition of games in terms of directional substance (essence as potential) embedded in a materialist casuistry. As Burke quotes Encyclopaedia Britannica, materialism "regards all the facts of the 
universe as explainable in terms of matter and motion, and in particular explains all psychical processes by physical and chemical changes in the nervous system" (Burke, 1969a, p.131). Notably, this is exactly how games, or certain isolatable elements of games, are seen to conjure states of mind in players. States of mind like happiness, motivation, engagement are the result of specific game-derived processes. The chain reaction is traced in the last sentence of the first passage, where a "system of principles, mechanisms, and rules" is said to activate a "system of rewards" that produces "predictable outcomes" which, in turn, "ratchet up a person's engagement, and increase happiness". This description of mental processes is too vague to be questioned, but it certainly creates the impression that human feelings can be treated as reliable mechanisms. Inadvertently, Penenberg also defines states of mind in terms of derivational substance. Rather than an abstract spiritual state, happiness is now seen as the predictable result of mechanical processes; therefore, akin to mechanism itself, it can be re-applied as further means to the ultimate end of "big dividends". Once again, every purpose becomes an agency, or agency transforms into an end in itself.

As observed, in Penenberg's text, and earlier in Hugos', games have been assigned an ambiguous pentadic function. On the one hand, the whole first passage lists the variety of ends game mechanics can be applied to (agency); on the other, they are also the scene that determines the actions or reactions of players. The agent, at first, concealed in the passive constructions of the first passage, is directly named in the second, where businesses, organisations, agencies and corporations are shown as the true locus of intention. It is by their "gameful design" that a total scene is created which then determines the rest of the participants, their motions and emotions. And if the fact that 
gamified subjects are under the influence of gamifiers is not emphasised enough through grammar, "gameful design" is straightforwardly defined as "the characteristics which make games fun and addictive". Indeed, it is one of the purposes of this book to explain how and why human feelings and behaviour should be treated as predictable and controllable mechanisms, but also how that control is achieved by limiting information access or suspended disbelief.

If emotions are to be reimagined as means, they need to be both spontaneous and reliable: spontaneous, because that is a measure for genuineness of emotion, and reliable, because this is a measure for efficiency of means. In one of the numerous examples he gives on successful applications of "gameful design", Penenberg quotes the game designer Michael Fergusson saying: "Fun is functional!" (Penenberg, 2013, p.101) A statement like that made by an expert practitioner - a game designer, compliments well the argument that human emotions can and should be used as vehicles to greater ends. This palpable paradox signals the attempt of the author to shift the conventional understanding of emotion by defining it in relation to impersonal mechanisms, i.e. by familial substance. The supporting rationale for that definition is largely provided by Csikszentmihalyi's notion of 'flow', which consistently reappears in the works of most gamification proponents. Penenberg describes flow briefly as "a mental state that game players enter when they're completely immersed in what they are doing and lose track of time" (2013, p.22). The psychological conditions facilitating the state of flow have been identified by Csikszentmihalyi and have been translated into "game mechanics" by Penenberg and other gamifiers, who credit them, along with the hormone dopamine and evolutionary adaptations for the addictive power of games. As he arrives at the conclusion 
that "our brains can be hacked (...) without our being aware" (2013, pp.23-24) Penenberg successfully explains how the spontaneous emotions of players are simply mechanical reactions to external stimuli, and their free actions are predicated by their initial submission to the game framework. The condition for the proper functioning of this proposed system of interactions is the unequal distribution of information. From the point of view of the gamifier or designer (agent), who originates the action, and have complete system knowledge, the behaviour of the player (agency) is a mechanism. On the other hand, the gamified subject, who is involved in the game, but has limited knowledge of its structure or utility, perceives oneself as an agent who freely chooses one's interactions led by one's own intentions.

For Penenberg, this asymmetry in information access of designer and player, is essential to the magical power of the game or its capacity to sustain a comprehensive illusion of the real. In fact, great design presupposes perfect deception. Using the support of scientists like Nick Bostrom, a philosopher at Oxford University and director of the Future of Humanity Institute and Rich Terrile, director of the Center for Evolutionary Computation and Automated Design at NASA, Penenberg suggests that "there is an almost mathematical certainty" that we are all inhabitants of the game simulation of a being from the distant future (2013, p.200). The argument that the creator of the world is a game designer from the future may be an impossible to verify hypothesis, but is definitely flattering for a game designer of the present, who is invited, by means of rhetoric, to identify with a God-like being. In the same motion, Penenberg equates reality with simulation, the present with the future, and human consciousness with inbuilt variability: 
In this book I explore how games and game design have been seeping into virtually every aspect of our lives and how they can unleash breakthrough thinking. Maybe, though, these games are actually seeping into our game. Instead of each of us controlling our bodies with our minds, our brains have been hooked up to one vast neural network and we're just role playing in a massively multiplayer fantasy game. After all, when you look at someone you're not really seeing that person. Your brain is simply interpreting light waves, converting them into data that it can interpret. You don't hear someone speak. Your mind processes sound waves that your ears pick up. (...) As it is, we exist one step removed from reality. Our connection with the surrounding world is already simulated. (Penenberg, 2013, pp. 200-201)

This passage envisions a human being constrained by the total scene of the game - a game with an unfamiliar creator, which we consider reality, because we have no recollection of the moment we've made the choice to enter it. All this is indicated by the use of present perfect tense and passive voice in the clause: "our brains have been hooked up to one vast neural network". The action, of which we are subjects, has taken place outside of our awareness, and has been executed by unknown agents. Human individuals are thus reduced to brains, senses, perceptions, processes of interpretation of received data, and the author achieves this by means of familial definition. "We" are made up of simple elements and processes and are substantiated in equivalence to those. "Your brain" (or mind) is simply a data emitter and receiver, which works according to impersonal laws of nature. Moreover, this lack of awareness of how it all began is not only guaranteed, but it is posited as the main condition to perceive something as real:

Of course, you wouldn't know you were locked in a simulation. "If the simulators don't want us to find out, we probably never will", Bostrom wrote in a 2003 paper titled "The Simulation Argument: Why the Probability that You Are Living in a Matrix is Quite High". (Penenberg, 2013, p.201) 
Hence, the "reality" Penenberg invites us to embrace is a perfect simulation with an impenetrable boundary between a known and an unknown. This unknown, which is taken for granted and desired, allows for all kinds of speculations. One of these is the brave imagining of the author that time is circular and what is created in the future we live now; another one is that the entirely determined gamified subject of the now is his own future game designer - God:

Terrile finds inspiration in the idea that we may soon have the technological wherewithal to create our own synthesized universes. That would mean that we, who live in a simulated world, have created a simulated world, whose denizens wouldn't know they're the product of our collective computing imagination. Now, what if our master designers also lived inside a simulation? Same for those who designed their simulation. Potentially you could have levels and levels of sims, perhaps millions of them. In that case, Terrile speculates, if there is a creator for our world, it is us, or at least an offshoot of us hailing from a distant future. In a sense, "we are both God and servants of God", he says. (...).

Now that's what I'd call the ultimate game design. (Penenberg, 2013, p.202) Penenberg posits several paradoxes of substance, which signal his rhetorical attempt for revision of definitions. One is expressed in the consubstantiality of reality and simulation, and another in the consubstantiality of future and present. The central one, however, consists in the claim that the simulated humans who are role-playing without being aware of the larger "game", can also be the designer Gods. In other words, the desire to become God-like is contingent on the acceptance of complete determination from outside. The proposed way to overcome these contradictions, which are constructed by the text itself, is to accept them as given, and then follow the author's advice towards a resolution. 


\section{The Act of Persuasion of Utilitarian Gamifiers}

The Dramatistic reading of these two authors, earlier classified as utilitarian gamifiers, brings into view the grammatical patterns that lay the ground for the interpretation of these texts. Strictly speaking, the participants in the gamification interaction are the gamifier, the game, and the gamified. As observed, subjects or entities placed in the category of the gamifier (experts, game designers, business owners, corporations, government) are consistently ascribed the pentadic function of agent. They initiate the act and invest it with purpose. Games have an ambiguous status, which fluctuates between agency and agent/scene. In other words, in relation to the gamifier, games function as means to an end, i.e. agency. In relation to the gamified subject, games appear as another agent, or scene. Finally, subjects or entities in the category of the gamified (end user, employee, consumer, fun, happiness) function as agency. The gamified human actor, whose capacity for autonomous, engaged action is emphasised in words, grammatically proves to be the recipient rather than the initiator of the act.

The attribution of grammatical roles goes hand in hand with the reaffirmation of a set of foundational definitions. The close circumference of the chosen vocabulary reduces 'reality' and 'nature' to economic principles of production and exchange, where "higher dividends" naturally substitute for "absolute purpose". Human beings, mental and psychological processes are governed by material forces, which can be isolated, studied and therefore reproduced by design. Games are a mysterious force in terms of potential impact (directional substance) and a reliable mechanism, when defined in adjacency with feedback systems and technologies (familial definition). The paradoxes of substance that surface in these definitions expose the rhetorical intentions of the authors to re-negotiate 
established conventions. Thus, the technologically enabled design of experience is justified by laws of nature; action is reduced to motion or passion; freedom - to necessity. It becomes apparent that gamification discourse exploits the very dialectical pairs Burke identifies as foundational to representation and substantiation. As Burke explains, "the paradox of the absolute figures grammatically in the dialectic, making for a transcending of one term by its other, and for the reversed ambiguous derivation of the term from its other as ancestral principle" (1969a, p.38).

In as much as these texts intend to communicate and persuade, they presume the participation of different kinds of entities, or classes between which an agreement is sought. On the surface, distinctions in status are overcome by inviting the reader - a layman, to identify with the expert gamifier. However, the very process of identification presupposes the acceptance of an established order - an hierarchical structure of difference put in place by the text. This hierarchy, that divides and harmonises in the same gesture is sustained and justified by a mysterious necessity. As Burke explains, communication happens between "estranged entities, and strangeness is a condition of mystery" (1969b, p.177). Thus, the hierarchy that is established in Hugo's and Penenberg's texts, consistently displays an order of determination, according to which the designer determines the game, and the game determines the player. This order is put forth as essential and necessary.

\section{Humanistic Gamifiers}

The distinction I make between utilitarian and humanistic gamifiers is provisional on the respective authors' declaration of purpose and scope of treatment. Utilitarian gamifiers, 
such as Hugos and Penenberg, treat gaming and games more strictly from a business perspective. Hence, entertainment is no longer an end in itself, but a vehicle to the achievement of increased efficiency and profit. In this group of authors, the original intent of the act of gamifying resides in the gamifiers, who then employ games and players as means to their ends. In other words, if the goals of the gamifier and the gamified subject coincide, that is because the latter was convinced to adopt the goals of the former by way of gameful deception or suspension of disbelief. Humanistic gamifiers, on the other end, consider games more broadly as a way of restructuring experience to the benefit of individuals and society at large. As they place more emphasis on the purpose of the player as an agent, or the 'necessary' coincidence of purpose between gamifier and gamified subject, their narrative becomes even more ideological than that of utilitarian gamifiers. Moreover, a closer look at the grammar of humanistic texts shows that, despite the difference in orientation, the hierarchical structure of participants remains the same as the utilitarian one. This is confirmed once again by the text of Jane McGonigal's Reality is Broken. I have selected this author to represent the humanistic group, because she is one of the first to suggest that the joys of games and entertainment can have practical application. McGonigal is an enthusiastic ideologue of gamification, who emphasises the democratic nature of games and its transferability to other areas of life.

\section{McGonigal: Reality is Broken}

Jane McGonigal, acclaimed game designer and researcher, deserves the title humanistic gamifier by virtue of her idealism, and unfettered belief that the fun, magic and fairness of game play can be transferred into trivial life tasks and routines. Her proposition is that 
since games make us happy, we should all be gamers, and not just as a way of escaping reality, but as part of everyday interactions and activities. She appeals for a future where "we use everything we know about game design to fix what's wrong with reality", and where "we live our real lives like gamers, lead our real businesses and communities like game designers, and think about solving real-world problems like computer and video game theorists" (McGonigal, 2011, p.7). For that reason, McGonigal is certain that she is addressing "virtually every person on this planet", because "everyone (...) will one day become a gamer", and games should "be something everyone learns how to design and develop, because they understand that games are a real platform for change and getting things done" (McGonigal, 2011, pp.12-13). This initial address already contains the central claims of the book: a) human happiness is contingent on the overlap between games and reality; b) game designers are the most experienced "happiness engineers" who should be designing reality as well; c) players can be active participants if they become designers. The familiar hierarchy that places expert gamifiers (game designers) at the top of the act structure appears at the very beginning of McGonigal's book, which otherwise emphasises the necessary connection between the autonomy of users and the success of a given gamification scheme. Her text exemplifies the thought process according to which human intention, originally conceived as intrinsic to the person, is conceptualised as externally motivated, and hence, redefined as means toward ulterior ends.

More than other expert gamifiers, McGonigal pays special attention to the psychology of the player and the game conditions which contribute to players' happiness. As she insists that players can use games to become active creators of their well-being, the grammar she employs contradicts the wording of her messages. In the 2nd chapter 
called "The Rise of Happiness Engineers", McGonigal refers to the work of a number of positive psychologists in order to suggest that human happiness is not just an abstract notion, but a concrete state of mind that has been studied and proven to result from a combination of mental procedures. Based on Mihaly Csikszentmihalyi's work, McGonigal describes games as "flow-inducing"16 "autotelic"17 activities, which allow us to "regularly achieve the greatest form of happiness available to human beings: intense, optimistic engagement with the world around us" (McGonigal, 2011, p.36). This state, which Csikszentmihalyi has also called "flow", is most commonly reached as a result of "highly structured, self-motivated hard work" that is freely chosen and "done for pure enjoyment rather than for status, money or obligation" $(2011$, p.36). Following the core teachings of positivist psychology, McGonigal proceeds to reveal "the four secrets to making our own happiness" by naming the four major categories of "intrinsic rewards" 18 to be obtained by engagement in autotelic activities (2011, p.45).

Ironically, this wordy emphasis on the active role of individuals in the creation of their own happiness is not supported by the grammatical structure of McGonigal's text. Even the very description of how and why we should be the masters of our mental wellbeing, does not assign "us" the pentadic role of an agent. The pentadic ratios dominating the text are found in the following series of quotations:

\footnotetext{
${ }^{16}$ The notion of "flow" refers to "the satisfying, exhilarating feeling of creative accomplishment and heightened functioning" as defined by Csikszentmihalyi in Csikszentmihalyi, M. Beyond Boredom and Anxiety: The Experience of Play in Work and Games. (San Francisco: Jossey-Bass, 1975), xiii. Quote found in McGonigal 2011, p. 35

${ }^{17}$ McGonigal writes: "The very act of what we are doing, the enjoyment of being fully engaged, is enough. The scientific term for this kind of self-motivated, self-rewarding activity is autotelic (from the Greek words for "self", auto, and "goal", telos)" (2011, p.45). In note 17 she attributes the coining of the notion to Csikszentmihalyi, Beyond Boredom and Anxiety (1975), 10.

${ }^{18}$ McGonigal uses "intrinsic rewards" to describe a deep sense of personal gratification - "the positive emotions, personal strengths, and social connections that we build by engaging intensely with the world around us" $(2011,45-50)$. This term appears consistently in gamification discourse to refer to the player's own desire for and inclination towards certain types of activities, which he/she experiences as gratifying.
} 
The prevailing positive-psychology theory that we are the one and only source of our own happiness isn't just a metaphor. It's a biological fact. Our brains and bodies produce neurochemicals and physiological sensations that we experience, in different quantities and combinations, as pleasure, enjoyment, satisfaction, ecstasy, contentment, love, and every other kind of happiness. And positive psychologists have shown that we don't need to wait for life to trigger these chemicals and sensations for us. We can trigger them ourselves through scientifically measurable autotelic activities. In fact, from a neurological and physiological point of view, "intrinsic reward" is really just another way of describing the emotional payoffs we get by stimulating our internal happiness systems. By undertaking a difficult challenge (...), we can produce in our own bodies a rush of adrenalin, the excitement hormone that makes us feel confident, energetic, and highly motivated. (....)

And if we provoke our curiosity by exposing ourselves to ambiguous visual stimulus, (...) we experience a rush of "interest" chemicals also known as "internal opiates". These include endorphins, which make us feel powerful and in control, and betaendorphin, a "well-being" neurotransmitter that is eighty times more powerful than morphine.

Few of us set out intentionally to trigger these systems. We don't think of happiness as a process of tapping strategically into our neurochemistry. We just know what feels good and meaningful and satisfying, and that's the kind of activity we'll do for its own sake. (McGonigal, 2011, pp.47-48)

What stands out in the above passages is that while "we" are presumably the "source" of our own happiness, "we" are not the originators of the acts that lead to it, but the passive receivers. It is "our brains and bodies" that produce neurochemicals that, in turn, trigger emotions that affect us in a positive or a negative way. Although "brains and bodies" are in the role of the agent here, i.e. they perform the act in the sentence, they are still not active in the dramatistic sense of intending an act. Rather, what we observe is a series of mechanical processes which trigger motions that spread like waves, producing further motions. Thus the individual is seen as scenically determined by "life" or natural forces independent of his or her free will. In the same vein of thought, "intrinsic rewards" which 
are previously defined as "self-motivated" and pertaining to the person's essence, are now substantiated through familial alliance with neurological and biological mechanisms. Hence, "intrinsic rewards" are simply intrinsic to the body, rather than originating from any spiritual or psychic core of the person.

The ratio scene:agent changes once "we" decide to take charge of the material processes that move us. In the examples McGonigal gives, "we" become the agent, and choose to "undertake a difficult challenge" (act) in order to get a "rush of adrenalin" (agency) that will make us feel confident and "highly motivated" (purpose); or "we" provoke our own curiosity (act) in order to get a kick of "internal opiates" (agency) that make us feel "powerful and in control" (purpose). However, in this case, everything seems to be uncomfortably reversed. The "difficult challenge" and the "rush of adrenalin" are now assigned the role of agency towards the purpose of "feeling confident and motivated" that belongs to the agent "we". In other words, the agent as the supposed locus of the action acquires motivation to act as a result of stimuli received due to having already acted, or else, the agent acts in order to feel motivated to act. Meanwhile, the action of challenging yourself doubles as the means towards getting a certain feeling for yourself rather than accomplishing any exterior purpose. At its final limit, this is an agent who acts upon oneself, uses oneself as means, and is one's own purpose.

The circular causality McGonigal encounters through the concept of selfgamification might be precisely the reason why "few of us set out intentionally to trigger these systems". Happiness, "well-being", feeling powerful are abstract notions that make for evasive goals, but are even harder to imagine as means toward ends. In other words, happiness is not an action that can be wilfully exercised. It is rather a consequence or a 
side effect of being involved in other actions. Therefore, while McGonigal points out that we don't usually "think of happiness as a process of tapping strategically into our neurochemistry", she mentions further on in the book that it would also feel inauthentic or dishonest to do so. This kind of conscious self-deception constitutes a paradox, for the transcendence of which, McGonigal and gamifiers suggest that we use games. Games are thus introduced as the stand-in for an exterior or otherness that mediates the relationship between ourselves as the agent, ourselves as the means, and ourselves as the end receiver of our own actions. This implies that "we" willingly submit to their mediating effects thus becoming an agent/co-agent determined by games as scene/agent. Such a reading of McGonigal's text is validated by her grammatical treatment of games:

Games, after all, are the quintessential autotelic activity. We only ever play because we want to. Games don't fuel our appetite for extrinsic reward: they don't pay us, they don't advance our careers, and they don't help us accumulate luxury goods. Instead, games enrich us with intrinsic rewards. They actively engage us in satisfying work that we have the chance to be successful at. They give us a highly structured way to spend time and build bonds with people we like. And if we play a game long enough, with a big network of players, we feel a part of something bigger than ourselves (...)

Good games help us experience the four things we crave most -- and they do it safely, cheaply, and reliably. (McGonigal, 2011, p.51) Games, of course, help put people back in control. Real gameplay is always by definition voluntary; it is always an exercise of our own freedom. Meanwhile, progressing toward goals and getting better at a game instils a sense of power and mastery. (McGonigal, 2011, p.149)

These excerpts, like the group prior to them, still emphasise the free choice of players, but one has to notice that it is games that act. They have clearly taken over the function of the main agent and are the locus of a whole sequence of negative and positive acts, such as not fuelling our appetites for extrinsic rewards, but actively engaging us, giving us structure and so on. Whether an agent or a co-agent, "us" is clearly the sufferer of games' 
actions. At least this is the case in all but the last quote, where the structure "games help put people back in control" suggests the presence of an additional actor, who is being helped by games to put people back in control. This brings the structure of interactions to its familiar form used in the texts of utilitarian gamifiers. Once again, games are the agency employed by an incognito agent toward the end of producing an effect on people. This particular sentence is especially telling, because it contains the paradox of substance that constitutes gamification. If games put someone in control, this suggests that this someone is essentially passive, i.e. not the original locus of the act. But if games are used by someone to put someone else in control, the locus of the act is already twice removed from those who are said to be in control. As if in confirmation, McGonigal points out that, it is "a sense of power and mastery", a mere impression, that games "instil" in us, not the thing itself. This brings us to her description of game designers, who are consistently in the pentadic role of agent:

Game developers today understand that games become hits and make money in direct proportion to how much satisfaction they provide and how much positive emotion they provoke - in other words, how happy they make their players.

Game designers and developers are actively transforming what once was an intuitive art of optimizing human experience into an applied science. And as a result, they are becoming the most talented and powerful happiness engineers on the planet. (McGonigal, 2011, pp.37-38)

These short passages reveal that if games were acting upon players, they do so on behalf of the game designers or as mediator's of the designers' intentions. The first sentence makes several claims at once. On the one hand, it makes it clear that designers (agent) "understand" how to make effective games. On the other, it spells out that effective games are recognised by the fact that they "become hits and make money" (purpose). It also 
points out that the condition or the means for the success of a game is the happiness of the player (agency). It appears that the dominant ratio here is agent:act, since game designers initiate an act in line with their purpose and choose the appropriate means for its achievement. It is interesting to observe, however, how the grammar of the sentence positions games at almost an equal ground with game designers. Although games appear in the subordinate sentence, and are by implication determined by the designers, they are also actors, and the verbs describing their actions, "provide", "provoke" and "make", are far more direct and active than the verb "understand" which places the participation of game developers on the receiving end of the interaction. Once again, games are given an ambiguous role which allows them to function as both co-agent and agency, and once again, the impact of the leading agent (game developers) is partially overshadowed by the mysterious, but impersonal power of games. The verb "understand" also opens the possibility for an alternative reading of the sentence. It might be suggesting that game developers are determined by the expectations of players, or that their final purpose is not to "make money" or accomplish other goals with games, but to make players happy. If this were the case, the whole hierarchy of relationships would be reversed.

Dramatistically, the question whether "satisfaction", "positive emotions" and "happiness" are the final purpose of game developers and players, or the agency, is the most central one in McGonigal's book and her answers oscillate from one extreme to the other. This hesitation in meaning describes and facilitates the transition from game to gamified environment and from play to gamification. Thus, McGonigal's insistence above that game designers have become the most powerful happiness engineers on the planet is another statement that can point both to the understanding of happiness as the goal, and 
the understanding of happiness as the means. Its meaning becomes more concrete in the larger context of her book, which is, after all, about applying games to the task of bettering the real world. As soon as games acquire a purpose outside of play itself, they become means, and so does the happiness they are said to create. Thus, by calling game designers "powerful happiness engineers" McGonigal does not emphasise their potential to create true happiness and stop there, but to employ positive emotions as instruments toward further ends. This becomes even more apparent in the following passages:

The modern history of computer and video games is the story of game designers ascending to very powerful positions in society, effectively enthralling the hearts and minds - and directing the energies and attention - of increasingly large masses of people.

The people who continue to write off games will be at a major disadvantage in the coming years. Those who deem them unworthy of their time and attention won't know how to leverage the power of games in their communities, in their businesses, in their own lives. They will be less prepared to shape the future. And therefore they will miss some of the most promising opportunities we have to solve problems, create new experiences, and fix what's wrong with reality. (McGonigal, 2011, pp.10-11)

Here, McGonigal directly points out that game designers' expertise has risen them to "powerful positions in society" as they have become the masters of the "energies and attention" of "large masses of people". Designers are the first to know how to "leverage the power of games" to various ends, hence they have the first say in shaping the future and fixing reality. At this point the hierarchy of determinations acquires a more complete form. From the bottom up, players are determined by bodily processes and chemical reactions, triggered by emotions, triggered by games, designed by game designers. The agent at the top are game designers, games and happiness are the agencies applied to encourage certain effects and behaviours in players. Interestingly, in order to become truly empowered, players are also encouraged to conceive of themselves as their own 
means (agency) and end (purpose), i.e. to be the source of their own happiness. In his Grammar of Motives Burke observes that "doctrines that reduce mental states to materialistic terms treat motion as motive" (1969a, p.32). As if to illustrate his point, McGonigal's text demonstrates how the adoption of "motion as motive", or else, the directional substantiation of the human being as determined by material processes leads to the treatment of oneself as an instrument of oneself. By extension of the same logic, humans and human experience in general also become instrumental. Gradually narrowing the circumference of her discussion, McGonigal leads the reader through a series of eliminations of dialectical opposites. In a neat sequence, consciousness is reduced to psychology, psychological processes are reduced to emotional responses, emotional responses are reduced to chemical reactions. At last, the "exercise of our own freedom" through games is eventually transformed and reduced to predictable determination by external forces and mechanical processes. This is precisely what Burke means when he explains that "the directional [substance] is also susceptible of conversion from "free" motion into the "determined"" (1969a, p.32). Dramatistically, the difference between action and motion is in the locus of motive. Since motive is internal to an act and external to motion, motion is necessarily determined.

In addition to reaffirming the instrumentality of games and happiness by means of grammar, the passages above also function rhetorically. McGonigal invites the readers to identify with designers or, at least, gamers in various ways. The pentadic ratios and distributions in her text clearly indicate that the greater the mastery of games, the closer one is to owning the act. In addition to that, there are numerous direct encouragements addressed towards the reader to enter the hierarchy of game relations by recognising their 
appropriate place. Along with previous inspired verbalizations of the utter necessity and benefit of games, the last example above, is a prophetic warning, verging on a threat, for those who refuse to do so. The "people who continue to write off games" McGonigal predicts will be at a "major disadvantage", because not knowing how to "leverage the power of games" will prevent them from equal participation in shaping the future, or fixing reality. Thus, as the counterpoint of the powerful gamifier emerges the detached from society, reality, and future person who refuses to 'play' along or be gamified. "Autotelic" activities as games may be, game playing is not voluntary, but the first legitimate form of participation in the society McGonigal envisions. It is an entry requirement to the lowest level of the hierarchical order constructed by the text that the reader has to accept for fear of being ostracized. Thus, only after accepting the proposed structure, and one's subordinate position in it, the reader is also prescribed the means to overcome one's subordination. Again, McGonigal's text illustrates the tendency of dialectical opposites to convert into one another. Her suggestion that empowerment comes at the price of subordination is both paradoxical, and to be expected. After all, notions like 'power' and 'freedom' have no meaning outside an established hierarchical structure.

Seen through the Burkean Dramatistic lens, McGonigal's humanism proves to be the ideological extension of the sincerely utilitarian gamification discourse of business gurus. The player who is the means of himself toward the purpose of himself (his own means towards his own happiness) is not any less utilitarian, or susceptible to utilization than the wholly determined and easily predictable gamified employee. The logic that treats happiness as a goal in itself, sought as a 'natural' and reliable effect of chemical 
reactions, also makes an instrument out of it. Since the freedom to play is, in fact, obligatory, and the being in control is a "sense" produced by external stimuli, the player's "intense engagement" or will to act is reduced to an automatically triggered motion or compulsion. Finally, the fact that McGonigal's scope of discussion is much wider than that of business entrepreneurs does not make games any less instrumental. Her goal may not be as pragmatic as improved work efficiency and increased profits, but it is far more ambitious. She aims at fixing reality and shaping the future by means of games.

\section{Rhetoric on Gamification}

If the promotional rhetoric of gamification constructs a doctrine that formulates the world "as is", theoretical discussions of the concept should open the ground for a more exhaustive, critical engagement with the problem. The external point of reference of theoretical discourse qualifies it as a kind of metalanguage or a logological perspective that should serve to insert the notion of gamification into a larger epistemological system. To explain the need for distinction between dramatism and logology, Burke himself refers to the traditional distinction in metaphysics between ontology and epistemology ${ }^{19}$. As he affirms, his notion of dramatism is ontological - "it stresses what we are: the symbolusing animal" (Burke, 1985, 89-93); meanwhile logology is epistemological because it deals with "words about words" (Burke, 1970, 14), and "the discriminations that we make by language constitute our realm of knowledge" (Burke, 1985, 89-93).

\footnotetext{
${ }^{19}$ Kenneth Burke (1985) Dramatism and logology, Communication Quarterly, 33:2, 89-93, DOI: 10.1080/01463378509369584 [online] Retrieved Dec 14, 2016 (http://dx.doi.org/10.1080/01463378509369584)
} 
By assuming the role of a commentator on the gamification proposition, and by treating videogames as a medium or a language, Bogost claims to write "words about words" or construct the logological perspective on gamification rhetoric. For this reason, it is interesting to observe whether his way of speaking, mainly in his book How to Do Things with Games, exhibits a difference in the hierarchical treatment of the gamified subject, games, and gamifiers. The forthcoming dramatistic analysis of Bogost's book demonstrates that even if he assumes the position of a theorist the construction of motive in his discussion is aligned with the hierarchical structures observed in gamification promotion rhetoric.

Ian Bogost's awareness of the persuasive power of words shows in his own critical approach. A game designer and a media theorist, he is one of the first critics of gamification who dubs it "exploitationware". In a famous blog-post from August 8, 2011 titled "Gamification is Bullshit", he exposes the hidden agendas that feed the popularity of the notion in a few straightforward sentences:

The rhetorical power of the word "gamification" is enormous, and (...) it takes games - a mysterious, magical, powerful medium that has captured the attention of millions of people - and it makes them accessible in the context of contemporary business. (...)

This rhetorical power derives from the "-ification" rather than from the "game". ification involves simple, repeatable, proven techniques or devices: you can purify, beautify, falsify, terrify, and so forth. -ification is always easy and repeatable, and it's usually bullshit. Just add points.

I've suggested the term "exploitationware" as a more accurate name for gamification's true purpose, for those of us still interested in truth. Exploitationware captures gamifiers' real intentions: a grifter's game, pursued to capitalize on a cultural moment, through services about which they have questionable expertise, to bring about results meant to last only long enough to pad their bank accounts before the next bullshit trend comes along. 
I am not naive and I am not a fool. I realize that gamification is the easy answer for deploying a perversion of games as a mod marketing miracle. I realize that using games earnestly would mean changing the very operation of most businesses. ${ }^{20}$

The exploitation Bogost objects against is of a rhetorical nature. Gamification, as he points out, is a game of words that allows the free association between concepts and areas of human activity previously kept apart. According to him the addition of the suffix ification to 'game' corrupts its true essence of a "mysterious, magical, powerful medium", and by transforming it rhetorically into a "simple, repeatable, proven technique[s]", makes it applicable to the mundane ends of business. In other words, Bogost objects to the ambiguous substantiation of games in terms of both predictable material processes and mysterious powers. His term "exploitationware" emphasises the presence of a hidden agenda to take unfair advantage of the 'good reputation' of games by using it as bait. It is clear that Bogost considers gamification a trickery on at least two counts: 1) it constitutes an imaginative mix-up of words, which intends the confusion of notions and their transformation 2) the games in gamification are "perversions" which can only produce short term effects. It is precisely because of his critical stance that Bogost seems likely to provide an alternative view and treatment of games and the gamified subject. However, while the author is offended by the misappropriation of the word 'game' and disputing the efficacy of gamification, he does not entirely deny that games can be used in a business context. Moreover, the insistence that games are a 'medium' already expands the concept of play to incorporate the additional meaning of "an intervening agency, means,

\footnotetext{
${ }^{20}$ Ian Bogost, "Gamification is Bullshit" [online] original date of post August 8, 2011 (Retrieved April $18,2016) \mathrm{http} / /$ bogost.com/writing/blog/gamification_is_bullshit/
} 
instrument" deriving from its original Latin use. ${ }^{21}$ Hence, Bogost's defence of the 'authenticity' of games does not seem to exclude their conceptualisation as means.

\section{Bogost: How to Do Things with Games}

How to Do Things with Games details Bogost's understanding of the significance of games outside of game contexts. In it Bogost argues that videogames should be understood as a "pervasive medium, one as interwoven with culture as writing and images", and with "valid uses across the spectrum, from art to tools and everything in between" $(2011$, p.7). To illustrate his point, he then describes in what ways the effect of games on us can be likened to that of established media such as language, literature, visual art, radio, TV, film. He also examines the ways gaming differs from and occasionally surpasses familiar methods of communicating and mediating experiences. The following is a more detailed definition of videogames, which exhibits the pentadic distribution and ratios the author works with:

Understanding the properties of a medium does help us better comprehend their nature and their implications. Videogames, the subject of this book, also have properties that precede their content: games are models of experiences rather than textual descriptions or visual depictions of them. When we play games, we operate those models, our actions constrained by their rules (...). On top of that, we take on a role in a videogame, putting ourselves in the shoes of someone else (...). Videogames are a medium that lets us play a role within the constraints of a model world. And unlike playground games, and board games, videogames are computational, so the model worlds and sets of rules they produce can be far more complex. (Bogost, 2011, p.7)

\footnotetext{
${ }^{21}$ medium (noun sg.) 3. an intervening substance, as air, through which a force acts or an effect is produced; 6. an intervening agency, means, or instrument by which something is conveyed or accomplished.

media. (n.d.). Dictionary.com Unabridged. Retrieved November 20, 2015, from Dictionary.com

website: http://dictionary.reference.com/browse/media
} 
It is interesting to observe how "we" - the players - are initially the agent who "plays" and "operates" games as "models of experience", but we are also "constrained" or determined by their rules. This appearance of reciprocal interaction between games and players, which implies the player's willing acceptance of the rules of the game, is redressed in the next sentence, where games take precedence once again. They are a medium that "lets us play a role within the constraints of a model world". The ratio agent:act (player chooses to play) is thus transformed into scene:agent ratio where games are the scene "constraining" the player. It is also hard to ignore the familiar emphasis on the fact that videogames are the kind of scene that directly acts upon us. It "lets us" do things or limits our actions as if fully animated with the choice or intention of a dramatic agent. This use of grammar shows that Bogost himself substantiates games in an ambiguous fashion. They function as a medium, therefore agency that we construct and use to our ends; but also as a scene or agent that constrains whoever assumes the role of the player. Furthermore, the consideration of games as scene signals a materialistic paradigm where everything is seen as the result of knowable and reproducible physical processes. The consideration of games as an agent assigns them an essence of a mysterious power, that cannot be entirely known, predicted or reproduced. This treatment of games is analogous with the one observed in the work of McGonigal and the two authors before her, or the concept of gamification at large. As Bogost himself recognizes, it is the kind of attitude that mixes the reliable simplicity and utility of -ification with the undeniable power and authenticity of the mysterious. 
Starting with the title, How to Do Things with Games, the reader is justified to imagine that the main objective of the book at hand is to prove that games can be understood as instruments. Granted that they are instruments or a medium, more questions follow. One has to ask, to what ends are they applied, and by whom. The titles of chapters seem to offer the quick answers to the question, not how, but what can we do with games, and although quite opaque and abstract, a lot of them point towards emotions rather than actions. The list of contents reads: "Art", "Empathy", "Reverence", "Music", "Pranks", "Transit", "Branding", "Electioneering", "Promotion", "Snapshots", "Texture", "Kitsch", "Relaxation", "Throwaways", "Titillation", "Exercise", "Work", "Habituation", "Drill" "Disinterest". Six out of all twenty chapters directly name emotional states, but even the rest, prove to treat specific emotions as the purpose or the consequence of game design. The following are quotes from the chapters named "Kitsch", "Relaxation", "Exercise" and "Promotion". They give a taste of Bogost's tone, intention, and treatment of the interactions between designer, game and player:

In Diner Dash [videogame], sentimentalism is accomplished by invoking the moral fortitude of hard work. It is a game in which a good work ethic, careful attention, and persistence always yield success. (...) The idea is one that appeals strongly to people. Despite received ideals of Puritanism and the American Dream, modern life is riddled with a strong dose of unfairness and random circumstance. By surrounding oneself with posters, or games that espouse ideals of control, the timeworn hope of pure will breads the wistfulness that makes kitsch appealing. (...) But for those who would seek such a purpose in games, one issue remains: kitsch was always meant to be displayed, to serve as a marker of an upward-looking bourgeoisie. (Bogost, 2011, "Kitsch" pp. 86-87)

Leaning forward is useful when the desired effect of a game is high attention and twitchiness. But what if we wanted another kind of experience from a game, from time to time at least: a relaxing lean-back experience - a Zen game. Of the few attempts to create relaxation in games, Journey to Wild Divine is most deliberate. It is marketed as a new 
age game, a game for wellness. Using a fingertip controller that measures heart rate and skin galvanic response, the player exerts control by attempting to manage this biofeedback. The player might have to regulate heart rate to balance a ball or aim a bow. (Bogost, 2011, "Relaxation" p. 89)

Exergames must inspire their players to move. But they also must inspire their players to want to move. In doing so, these games both adapt existing rituals and practices from other domains, like sports, and establish new ones unique to videogames. (Bogost, 2011, "Exercise", p.114)

While advergames promote a company and its products, promogames offer an incentive to consume the company's goods independent of the game's representational properties. (Bogost, 2011, "Promotion", p.68)

The above passages demonstrate the range of applications possible for videogames. The chapters "Kitsch" and "Relaxation" focus on successful strategies for design of emotional states. As the target of design, human emotions are familially defined as a commodity ruled by the market logic of supply and demand. "Sentimentalism", "wistfulness", "high attention", "twitchiness" or "relaxation" are sentiments designated as the various possible purposes of the act of design, or play. In turn, videogames are again the agency applied by an unspecified agent. Whether designer and player coincide or not, it is the purpose here that determines the act (purpose:act ratio), i.e. the appeal of sentimentalism, wistfulness or relaxation justify the design of games that address the said emotional needs. The purpose also determines the means (videogames), and, consequently - the agent (designer/player), who has chosen them in accordance to the purpose or submits to his own means (designs or games) to receive the pleasurable experiences that were the original purpose. Bogost's use of grammar thus leads him to the circular causality already observed in McGonigal's conceptualisation of gamification, where the agent acts upon herself, uses herself as means, and is her own purpose. This transpires in Bogost's example of the relaxation 
game Journey to the Wild Divine, where the player regulates his heart rate to balance a ball that indicates that he is calm. To play the game successfully, one needs to be already calm, however, it is the game that measures, gives evidence, and takes credit for causing the player's calmness. Here, once again, games as a mediator or a medium, absorb the responsibility of the agent to relieve one from the embarrassment of deliberately designing one's own emotions. Since evolutionally emotions are part of the human response system, they have to emerge in reaction to external stimuli in order to feel genuine. For this reason Bogost, like McGonigal and the utilitarian gamifiers before them, use the "mystery" of games as justification to promote them to the pentadic role of agent. A good example for that is the passage from the chapter "Exercise", where videogames incite movement, as well as desire for movement in the player. This double emphasis means to suggest that once emotion is tied to action it becomes consistent and predictable - a behaviour. In other words, games as the scene determine the behaviour of the player as the agent. Moreover, the word "must" indicates that someone external to the interaction game-player intends and prescribes what games should or should not be able to do. With this, the hierarchy inherent to the gamification concept is reaffirmed by the standard sequence of dependencies according to which the gamifier determines the game, and the game determines the gamified subject. The definitions of advergames and promogames in the last quote above single out one such occasion when the purpose of games is entirely pragmatic - the promotion of products or brands. The gamifier, still partially concealed behind passive constructions, can be recognised in the business owner or the corporation.

As observed, in Bogost, like in McGonigal, the pentadic role of videogames shifts from agency to scene or agent, while human emotions and behaviour function as purpose, 
but are treated as agency as well. In short, appointed purposes transform into agencies towards further purposes that often remain undisclosed. In line with McGonigal's proposition that we should all learn to design games, Bogost seeks the saving grace of gamification in the idea of a player who is equipped to act through games knowingly. His conception of the agent player is based on J. L. Austin's theory of language as a performative action and comes closer to Burke's definition of the dramatistic agent as the original locus of the act. In the following passage Bogost spells out both the failure of gamification and how it should be addressed:

When a game performs an action without the player's understanding of its implications, it confuses performativity and exploitation. "I do" is a meaningful performative utterance because bride, groom, and witnesses all fully understand its implications. But the ESP Game veils the possible implications of image tags, for example, as tools of surveillance as much as for image searches.

Performativity in discourse couples speech to real-world action rather than representation. Performativity in videogames couples gameplay to real-world action. (...) In addition, the player must develop a conscious understanding of the purpose, effect, and implications of his or her actions, so that they bear meaning as cultural conditions, not just instrumental contrivances. (Bogost, 2011, pp. 121-124)

According to Bogost a game becomes performative under two conditions: the first one is that the action within the game produces results outside the game; and the second - those results are well understood and intended by the player himself. If the player's actions happen to produce effects he did not intend, they would fall under Austin's definition of "unhappy performative". Judging on the majority of examples Bogost's book presents, performativity through games is more of a good wish rather than an actuality. Moreover, if the "mystery" of games has so far been affirmed as a necessary condition for the efficacy of gamification, the idea of conscious self-deception or conscious self-distraction 
by means of games not only constitutes a contradiction in terms, but defeats its own purpose. The performative games Bogost describes are honest about the sought results and collectively agreed upon, but may be perceived as too 'real' or adding more rules to the work place rather than fun. The game $A t t e n t^{22}$, for example, ascribes a monetary value to employees' attention by attaching a price tag in "serios" (made-up currency) to interoffice emails. Employees are thus alerted to the greater importance of certain messages, and are encouraged to give them priority since that earns them more serios. In turn, to have their emails answered they have to spend out of their personal reserve, and the cost for emailing managers is higher than that for emailing co-workers. Attent may be considered a game because it introduces novelty into a routine environment, and a fresh perspective on the time cost of email correspondence, but participation in it can hardly be voluntary. If one chooses not to play, one risks exclusion from the communication loops at the company and that would eventually compromise one's performance. Once an obligation, the so called game is quickly reduced to a fanciful way of introducing new regulations at the workplace. The initial 'fun' and novelty of the serios script currency is likely to wear down and turn into another complication to the tedious task of handling email. Although an ingenious idea in some ways, this game is a structural filter for company procedures which has been applied there by the management in a patronizing, disciplinary gesture. It is also noteworthy, that even as Bogost gives an example of a performative game, where the player should be the one intending the act and its consequences, the grammar he uses still places games in the dominant pentadic role of

\footnotetext{
${ }^{22}$ Attent is a game made by Seriosity - an enterprise solutions company founded by Byron Reeves and J. Leighton Read authors of Total Engagement, Harvard Business Press 2009.

(http://www.seriosity.com/attent_demo.html)
} 
scene/agent. It is still the game that conditions the player's behaviour, as well as consciousness:

Time management isn't the answer, less email is. And one way to reduce the email people receive is to make it more precious to send. Attent tries to do exactly this, making email more expensive to send, or at least making people more deliberate about how they do so. (Bogost, 2011, p.122)

The fact that employees turned players cannot have choice in the matter whether to play or not is enough to cast doubt on the proposition that Attent is a game, but even more so on the proposition that it is performative play. According to Austin, one of the conditions for a successful performative utterance is the sincerity of the person speaking. To give a promise without meaning what you say constitutes an "infelicity" ${ }^{23}$, and so should playing out of obligation.

The examination of Bogost's texts reveals that, although he begins with a stern critique of the concept of gamification, his own description of videogames as a means of communication does not differentiate by far in terms of grammar use or even content. The author's effort to conceptualise videogames as a "language" anyone can master, is a rhetorical device that seeks to attract a larger following to the idea of their egalitarian or, at least, neutral nature. While, it is Bogost's intention to demonstrate how videogames,

\footnotetext{
${ }^{23}$ In his book How to Do Things with Words, J.L. Austin explains that there are a number of conditions that need to be fulfilled before the utterance of words can successfully coincide with the performance of an act. There needs to be "an accepted conventional procedure", which should be invoked under the "appropriate circumstances" and executed by all participants both "correctly" and "completely". The persons "participating in and so invoking the procedure must in fact have those thoughts and feelings" for which the procedure is designed, and must intend to and behave according to their pronouncements subsequently. His most famous and telling example of performative utterance is saying "I do." in the context of a wedding ceremony. If any of the above conditions are not met, then the performative utterance is "unhappy" or "infelicitous" (pp.13-15). In addition, Austin points out that speech-acts, like all acts, can be considered "infelicities" if performed "under duress, or by accident, or owing to this or that variety of mistake, say, otherwise unintentionally" (p.21).

Austin, J. L. (1975) How to Do Things with Words. Second Edition, Edited by J. O. Urmson and Marina Sbisà, Harvard University Press, Cambridge Massachusetts
} 
like many familiar art forms before them, successfully answer our need for emotional stimulation, or experimentation with alternative life scenarios, his narrative is also instructive, pointing towards existing challenges in game design, and obstacles before the incitement of particular emotions. Established art forms already offer access to emotional states or experiences without the disadvantage of actual consequences in material reality. However, a novel or a film does not aim to micromanage the reactions of its audiences. According to Bogost, to design videogames is to design for specific emotions (or the experiences that trigger them) that are desired or pursued by players, and utilised by gamifiers to ends reaching beyond those of the individual player or play itself. Human emotion, turned into a commodity, is shown to be the purpose of design and the instrument of design applied towards subsequent greater purposes. Bogost carefully avoids pointing at a single grand mission that games can be applied to, but he certainly propagates their importance, versatility and impact onto the real world. As he refrains from ethical judgements on the larger implications of the gaming medium or performative play, he still uses the grammar of promotional gamification discourse that places the player at the bottom of the hierarchical structure it conjures and affirms. Videogames are a medium because they are rhetorical. They certainly communicate and enact the intention of the designer or the gamifier, but not necessarily that of the gamified subject or player. Therefore, to suggest that they are a medium that allows equal participation is to work towards supplying the needed ideological justification for a practice Bogost himself has called "exploitationware". 
The Dramatistic and rhetorical analysis of the texts of Hugos, Penenberg, McGonigal and Bogost illuminate not only the representational value and content of their respective messages, but their function as motivated action. As representatives of the so called "utilitarian" type of gamifiers, Hugos and Penenberg lay the ground for the conceptualization of games as means. The utilitarian perspective, whether business oriented or not, has a directness of tone and simplicity of intention that facilitate the identification of participants, roles, and acts in the gamification interaction, and illuminate their organization in a comprehensive structure of dependencies. Both authors treat games as complex systems of interactivity, where the gamified subject is submitted for processing. Games act upon the player, mechanically, as an environment driven by predictable physical processes would; or they act "mysteriously", out of a will of their own - a stand in for the will of the gamifier. Thus, the locus of the defining act of the gamification interaction is in the gamifier, who uses the game as means to transform the player into means towards some end. Or else, the so called 'voluntary' participation of the player is grammatically reduced to automatic motion. For Hugos, whose narrow circumference of treatment defines reality as economic conditions, the worthwhile purpose of gamification is the improved efficiency of employees and increased profits of the business enterprise. For Penenberg, whose circumference of treatment is so wide as to allow him to equate the everyday world with a game or a simulation, the worthwhile purpose of gamification is the successful design and management of human behaviour. Penenberg's motto, "Fun is functional!", is the best indication of how human feelings, or states of mind are consubstantiated with mechanisms. Rather than the ultimate purpose, now happiness is reduced to an agency - the instrument towards some further end. In 
Penenberg's universe, where 'the real' is sustained by the perfection of the simulation, the free choice and action of the gamified subject is a matter of perceptual illusion hinged on carefully limited access to information.

In contrast to the more straightforward distribution of intent and power in utilitarian gamification texts, humanist gamification writing attempts to rehabilitate the gamified subject. For that reason, its audience is wider, addressing those who are meant to inhabit the gamified environment, as well as those who are meant to design it. The persuasion technique humanist writers like McGonigal use is to invite the gamified subject to identify with the gamifier, or better yet - to recognize oneself as a self-gamifier. Working towards the psychological justification of such a proposition, McGonigal arrives at the formulation of a paradoxically cyclical self, who is defined in terms of "matter and motion" and functions as its own resource, instrument and purpose. Similarly, by defining games as a medium of communication, Bogost emphasises their democratic, all-inclusive nature, but also stresses their applicability to the creation of experiences and the evocation of emotions on popular demand. Whether they are conceptualised as the end goal or the incentive for further desired behaviour, human emotions acquire the quality of market commodities, and like commodities, happiness, fun, and spontaneity are locked in a perpetual limbo between the pentadic role of agency and the pentadic role of purpose.

This transformation of the end goals into further means, as Burke suggests, are the predictable giveaways of a materialistic, pragmatic casuistry of the neo-liberal kind. In that respect, it would not be too far fetched to claim that considered holistically, the effects of gamification discourse are ideological in function, and the ideology it constructs 
places human emotion on the conveyor belt of mass-production along with play and experimentation.

The close examination of these four texts on gamification legitimates their consideration as representative of a growing discourse or an integrated logological system with its own program of action and real-life implications. As a way of speaking, it constitutes a whole new framework of thought with its foundational definitions and hierarchies that assert themselves as necessary and true. To further evaluate their organizational capacities, it would be useful to study not only promotional rhetoric and theoretical discourse on gamification, but also the discourse or language of applied gamification. For that reason, in the following chapter I will look at the website of Pymetrics (recruitment company which uses games for profiling job seekers) as an example of applied gamification rhetoric. 


\section{CHAPTER THREE}

\section{Pymetrics as an Integral Reality Artefact}

The overview of gamification theory, or rhetoric about gamification through the Burkean Dramatistic prism, exposes its value system, foundational definitions, and mysteries that taken all together comprise a comprehensive worldview. As a way of speaking, gamification theory is an act with a purpose and direction, it forms an attitude. This attitude is then realized in cultural forms such as actual gamification products and applications that organize concrete social practices. If gamification theory forms an attitude, or a prescription for action, gamified applications, like Pymetrics, are its realization. Drawing on Baudrillard's extensive commentary on symbolic structures as foundational to society's way of life and belief system, I examine the practice of gamification as framed and reflected by existing gamification artefacts.

Admittedly, by treating language as action and grammar as a way of selecting a reality, Burke studies the motives of the participants in a culture in correlation to their worldview. By studying the structure of the sign and signification, Baudrillard diagnoses society as a framework of symbolic interactions permitting only the exchanges specific to its form. Therefore, while the first instructs that gamification rhetoric is a motivated act, 
the second observes the effects of this act on the fabric of experience. On the background of Baudrillard's notions of the code, and integral reality, the concept of gamification and its applications, appear as the symbolic strategy of our time for handling "the radical illusoriness of the world" (Baudrillard, 2005, p.32). Therefore, I claim, that the existence of gamification as a particular relationship to knowledge, self and time justifies the use of Baudrillard's integral reality as a descriptive term for the contemporary condition. Conversely, the application of gamification as a design principle is bound to produce integral reality artefacts.

To prove that gamification applications are integral reality artefacts, I analyse the gamified recruitment application of Pymetrics, and demonstrate how its rhetoric is derived from a worldview analogous to Baudrillard's integral reality. The suggestion that computer games can assess personality traits or proclivity toward a specific career, directly implies the contingency between virtual and embodied experience. Pymetrics assumes the translatability of in-game action into behaviour patterns, and the game scores into evidence for inherent potential and future performance of users. Therefore, to function as promised, gamification applications merge fact and artefact, blur time distinctions, and equate experience with its virtual representations. In other words, they exhibit the characteristic effects of what Baudrillard calls the "total substitution" of reality with virtuality, or what his translator Chris Turner paraphrases as a "programme of total production which itself supplants the world, (...) turning it wholly into known" (Turner, 2005, p.9).

My method of measuring applied gamification rhetoric against Baudrillard's framework of terms involves the analysis of the content of Pymetrics' website, my Trait 
report and my first-hand experience of the games. Inhabiting the perspective of a direct participant or user of a gamified application is the only way to access the bits of applied gamification rhetoric reserved for and specifically addressed to users. To the extent that my analysis of the Trait report and games is also an account of my perception and reaction to my own Pymetrics evaluation my analysis involves autoethnographic elements. According to Ellis, Adams and Bochner, "Autoethnography is an approach to research and writing that seeks to describe and systematically analyze (graphy) personal experience (auto) in order to understand cultural experience (ethno)" (Ellis et al., 2011). Although contentious (Coffey (1999), Sparkes (2000), Holt (2003)) ${ }^{24}$, this self-reflexive mode of analysis incorporates the researcher's subjectivity and bias as an inevitable part of the research process and allows me to treat my personal account of a gamified subject as indicative of the general effects of gamification. Moreover, a theoretical discussion with elements of self-reflection is better aligned with Baudrillard's aphoristic style of theorising characteristic of his Cool Memories series. With its melancholic mood, dated entries, and increasingly fragmented narrative, the series follows the format of a journal that displays the traces of fading personal memories. This is Baudrillard's way of avoiding the totalizing claims of traditional theorizing and allowing meaning to emerge through snippets of random thoughts and poeticisms that are endlessly interpretable, and never complete. This type of "theory fiction" is his way of showing "the self-evident in all its

\footnotetext{
${ }^{24}$ Since autoethnographic evaluation usually takes the form of self-reflection, it is often criticised for being self-indulgent and self-interested rather than critical (Coffey, 1999). Its qualitative and interpretative criteria of evaluation are not standardized, and therefore, hard to compare to the conventionally traditional requirements of qualitative and quantitative research for objectivity, reliability and reproducibility of findings. For this reason, autoethnography is still a contentious method, often considered at the boundaries of legitimate academic research (Sparkes 2000; Holt, 2003).
} 
glory, at the expense of truth, with contemptuous disregard for reality" (Baudrillard, 1996, p.17).

The product of Pymetrics can be treated as a cultural artefact, because it is a social media phenomenon. More importantly still, the product of Pymetrics is an integral reality artefact because it treats scientific representation as identical with physical reality, the subject as a digital trace, and time as ahistorical. The word "science" appears on Pymetrics' website as a sign of unquestioned authority. "Science" is a foundational notion, which authorises actions and claims, while its own content remains unexamined, and beyond doubt. In a world organized by Baudrillard's "code", the name proves the existence of the thing it names. My analysis of Pymetrics' website shows that "science" has become a sign of itself - both a symptom of and a justification for the equation of representation with experience. The same "scientific" approach also transpires in the construction of the games as assessment tools. As a consequence of the equivalence placed between the real and its sign, the subject is identified with and constituted by her digital trace. The structure and language of the Personal Traits Reports demonstrate how the virtual real-time actions of players who respond to the demands of the moment, are then understood as a pattern with a stable meaning. In that sense, events and actions taking place in virtual real-time signify and supersede embodied experience. Time is condensed into 'real-time' where the present is consistent with the future since both are equally known. Thus, while the behaviour of the player in the game is generalized into a sign for the person, the game environment itself functions as the condensed version of all possible contexts and environments one could ever be acting in. 


\section{Pymetrics in Context}

Pymetrics is a New York City-based company which combines "neuroscience games and big data to help companies get better at hiring" ${ }^{25}$. The company was established in 2013 by the MIT graduates Frida Polli (CEO) and Julie Yoo (chief data scientist), and is recognized as one of the pioneers of gamification. Presently, Pymetrics carries $\$ 8.85$ million in funding from sponsors like Khosla Ventures, Mercer, Randstad and \#builtbygirls. The Pymetrics games, which are available online and on mobile devices, "assess 50 different cognitive, emotional and social traits of the person playing". Based on the accumulated data, the Pymetrics algorithms, then, determine the ideal career for the job seeker and select the best talent for companies. Their client base consists of more than 25 companies among which is consumer packaged-goods giant Unilever. Pymetrics belongs to an expanding group of companies and government agencies which use gamification for recruitment, user engagement and goal tracking. Another example of a game-based recruitment company is Knack which was founded in 2010 and claims to blend "mobile games, cutting-edge science, and smart technology into an awesome experience that gives people from all walks of life the power to discover their talents and reach their potential for success" ${ }^{26}$. The U.S. Army has been using games for training purposes for decades, but by 2008 it started sending the transportable "Virtual Army Experience" game units to malls and public events to attract new recruits. Other recruitment games are Reveal by L'Oréal, Trust by Danone, and Moonshield by Thalès, which test the professional and cognitive abilities of aspiring employees by immersing

\footnotetext{
${ }^{25}$ Pymetrics was most recently featured in Upstart 25 in 14 th place among start-ups that will change the world. I am quoting from the article on them on CNBC's website retrieved March 29, 2017 (http://www.cnbc.com/2017/02/28/upstart-25-pymetrics.html)

${ }^{26}$ Quote from Knack's website. Available online at: https://www.knack.it/ [Retrieved 23 June, 2017]
} 
them in virtual environments build in the likeness of real-life work situations. In addition to recruiting and training, games are also used to keep track of employee success rates (Chore Wars, SCVNGR), customer loyalty (Samsung Nation, Teleflora Rewards), and personal goal achievement (Recyclebank, Mint.com) (Chou, 2017). The entertainment value of all of these games, or rather gamification tools, may vary, but what they have in common is that they strive to connect virtual experiences and actions with real life effects. They are designed to evaluate and manage the behaviour of a gamified subject, and their authority rests on data algorithms which function on the assumed equivalence between the sign and its referent. Due to these underlying commonalities, I expect that their treatment and effects on the gamified subject are essentially similar to those of Pymetrics.

\section{The Science behind the Pymetrics Product}

As a company which founds its business on gamification, Pymetrics faces the important challenge of not only advertising its services, but legitimating its innovative approach. Undoubtedly, games offer the benefit of being a popular attraction, but their utility or relevance in a business setting has yet to be supported by conventionally acceptable arguments. In other words, the transferability of games' impact in the physical world has to be verified by affirming the affiliation of virtual games with everyday physical reality. Pymetrics claims to have constructed and proved this direct transference between the lived and the imaginary with the help of science. Hence, neuroscience and the algorithms based on it are the reality element implanted in the imaginary of games, due to which the effects of games can be expected to reach further than the field of the imaginary. 
Pymetrics' website builds its argument with the use of short declarative snippets, which appear as slogans one at a time as one scrolls down the page. The banner statement on their title page is in an imperative voice. It reads: "play games to find your first job or best fit career". This is followed by: "Resume review is biased, our algorithms are not: pymetrics is blind auditions for career assessment + hiring". The website offers several tabs for further investigation: for job seekers, employers, career centers, the science, the games, in the media. Expectedly, the tone and arguments used for their respective types of audience differ, but scientific justification is central to all addresses. In the section for job seekers, the first slogan reads: "Play games based on decades of neuroscience research". The section for companies and employers opens up with the statements: "Neuroscience games asses unbiased cognitive and emotional traits in people. /Algorithms developed from successful employees' game play determine which traits predict success in over 100 careers and companies."

The emphasis on the role of science in the creation of Pymetrics' games has an important function. It brings Pymetrics' claims within the realm of convention and popular belief. Neuroscience is trusted to place the alleged practical uses of games beyond further doubt. Yet, the website's link devoted to the science behind the games does not offer very much additional or concrete information. The opening banner of this tab states: "More than just games: Our assessments are rooted in decades of scientific research". This claim is not supported by examples of studies, external links, or a list of publications. Instead, the information that follows is organized in a question-answer format and it lets the site visitor know that the co-founders Frida Polli, PhD, and Julie Yoo, $\mathrm{PhD}$ have "combined their decade of neuroscience research experience at Harvard 
and MIT to bring neuroscience assessment and data science to the world of career assessment and recruiting". The pymetrics games are then described as "science exercises that were developed by the global neuroscience community over several decades of wellestablished neuroscience research". What the games assess, rather than how, is the focus of the next snippet of information:

The platform consists of 12 games that assess 90 key cognitive and personality traits. Together, these games provide a snapshot of a person's unique characteristics. The 12 required games and 8 optional ones were selected by the founders as tasks that would most reveal the traits that would be relevant for assessing career fit. They were also chosen to be tasks that are not known for gender or ethnic bias. (Pymetrics, 2013)

The above several quotes exhaust the most relevant references to "the science" that should back up Pymetrics claims and the service they offer. However, with the use of general terms and statements like "science exercises", "global neuroscience community" or "decades of well-established neuroscience research" they prompt more questions than provide answers. On a closer look, the section on "the science" repeats terms like "neuroscience", "research", "assessment", "cognitive traits" multiple times, not to unpack them, but to give more weight to its announcements on what the games can do. With this, the company demonstrates that, on the one hand, it does not expect prospective users to question the scientific foundation of its product, and on the other, it does not trust that users will be interested or capable of understanding even a digest explanation of the scientific research or methods at work. Thus, the only proof given for the authoritative presence of science is the word science itself.

Taking for granted the happy marriage of scientific fact and playful imagination, Pymetrics insists that algorithm based games should be trusted to succeed where humans 
have not. Therefore, it first prompts prospective job seekers with the following sequence of imperatives:

Play games based on neuroscience research!

Discover your unique cognitive and emotional strengths!

Explore careers where your inherent traits lead to success!

Get recruited by companies you are perfect for! (Pymetrics, 2013)

On the next link named 'For Employers', it addresses prospective hiring companies with more imperatives:

Find and engage the best candidates anywhere!

Use our unbiased scientific games and algorithms to identify talent!

Recruit top-notch candidates that fit the cognitive and emotional profile of your successful employees! (Pymetrics, 2013)

And finally tells us what science and technology do better than all of us at 'The Science' link:

The pymetrics games are a series of neuroscience assessments that evaluate 50 cognitive, emotional, and social traits. Based off of those traits, we can recommend careers and companies where you are predisposed to succeed at.

Neuroscience games asses unbiased cognitive and emotional traits in people. Algorithms developed from successful employees' game play determine which traits predict success in over 100 careers and companies.

Algorithms are gender, ethnic and pedigree bias-free so that everyone has equal opportunity at all careers. (Pymetrics, 2013)

The first set of invites make it clear that, by participating, the individual user will learn something definitive about her "natural" tendencies and character that she did not know before, and that is 'objectively' true, i.e. confirmed in reality by science. Based on this personality blueprint, the user will then be matched with fitting careers, or be recruited by 
companies. And although the verbs "play", "discover", "explore" imply free intentionality, the person is directly told what to do, and promised positive outcomes. Neuroscience, according to Pymetrics, unveils the user's affinities and capacities with a concrete certainty no individual is capable of when pondering the mystery of oneself. Therefore, algorithms, rather than personal preferences, should be the more reliable guide to personal success and happiness; and the right and responsibility of making choices should be traded for the promise of the safe bet.

In a similar fashion, Pymetrics' address to companies, urges the abolition of established hiring practices and criteria for the sake of unknown assessment mechanisms that should be more precise, efficient and far-reaching. Such a proposition holds only on the background of an overarching belief in the powers of modern science and technology. It is neuroscience games that can "assess" reliably cognitive and emotional trends, hence can "predict success" in various careers, and are also "bias-free". In a word, the collective wisdom of human science is more authoritative and trustworthy than the know-how of any human individual. From this standpoint Pymetrics offers a tool that users apply to their own ends, and yet to do that, they need to submit to it blindly, apply it on themselves resigning to the role of passive participants led and framed by mysterious mechanisms. This is the familiar paradox of technology that inevitably puts forth dependence as the condition of being in control.

If the reliability of computational algorithms is taken for granted, the only concern remaining is how to not "game the game", or otherwise ensure that people do not understand the workings of the algorithms and cannot manipulate the results. This is the kind of reassurance on this subject Pymetrics posts in the FAQ section of its website:

Can you fake the games? 
Pymetrics games are very difficult if not impossible to fake for several reasons. First unlike questionnaires, a person can not figure out what the right answer is and so it is hard to know how they change their behaviour to get the right outcome. Second, even if a person knew what a test was measuring and how it mapped to a career algorithm and tried to game it, we measure behaviour at the microsecond level, which is not a level under conscious control. (Pymetrics, 2013)

The mechanical tool's impenetrability to personal motives guarantees its 'objectivity' and efficiency, because science and technology are conventionally seen as the more reliable bridge to reality than individual perceptions and interpretations. Thus, scientific knowledge originally produced by persons has to be automated and turned into a 'selfsufficient' algorithm locked to the further intervention of persons, so that it can be trusted as truth. The depersonalisation of science is part of the process of its mystification that on the one hand makes its authority indisputable, and on the other, makes it into a sign of itself.

The belief that human science is a confirmation or an extension of reality itself is yet another example of what Baudrillard calls "this superstitious belief in - this hysteresis of - the 'real"' (2001, p.18). Pymetrics' treatment of science as the real itself, rather than an attempted interpretation of it, is a performance or the kind of "imposture" Baudrillard considers definitive of our times. One of the consequences of this hysteresis of the real in the case of Pymetrics, but also in general, is the equation of the subject with his virtual presence or data trace. Once again, to suggest that a player's performance in a set of neuroscience games can reveal one's behavioural traits, cognitive capacities, core personality features, is to make a number of assumptions. The first one is that there is an integrated self or a kernel of personhood, which persists as a constant; the second is that this constant or personality blueprint may not be knowable to the person herself, but is 
obtainable by means of science - i.e. 'scientific' games; and finally, that the virtual trace she creates in the process of play directly translates into a certain kind of embodied behaviour. These assumptions facilitate and shape the experience of the player, which I examine further by taking on the role of a Pymetrics user or job-seeker.

\section{The Treatment of the Subject or Pymetrics as a Self-relation}

Pymetrics seeks to disentangle the 'mystery' of human potential with the help of the familiar scientific procedure of taking apart the object of study into its composite elements. Human behaviour, they claim, may be complex, but it is not unpredictable, it may seem spontaneous to the person acting, but it forms a pattern to an external observer who has the patience and the right tools for collecting data. For that reason, user personality is compounded into a diagram of inherent potentialities solidified in the Traits Report as the proper description and proof of the player's presence and essence.

Pymetrics' gamified algorithms, however, are themselves systems of signification, which forget their interpretative origin and take for granted the exact translatability between various types of signs, as well as the exact equivalence between facts of embodied reality and the signs that represent them. It is precisely the ostensible translatability of the imaginary into the real that guarantees the authority of their claims. Thus, the convoluted, but mechanical process of the games' algorithm is trusted as the guardian of scientific objectivity against subjective interpretations or intentions. The following analysis of my personal experience of the Pymetrics games traces more closely the subject's submission to a framework of mechanical processes and beliefs that reconstitute it into a sign of itself. 
The standards of "scientific objectivism" shape the treatment of the Pymetrics user in the following ways: 1) the subject is examined as a composite of basic, analysable elements; 2) the algorithm-based games serve as the criteria and the tool of the subject's legitimation. As Baudrillard reminds us, the human is continuously framed by the technologic:

Behind every television and computer screen, every technical operation which confronts him daily, the individual is analysed in return, function by function. He is tested, experimented on, fragmented, harassed, summoned to respond: a fractal subject doomed henceforth to be disseminated in the networks. (Baudrillard, 2001, p.50).

My interaction with the Pymetrics gamified algorithms and the resulting personal assessment illustrate, although in a limited way, the process and effects of the submission of the gamified subject to technological criteria of validation.

By signing up for their service the user of Pymetrics agrees to play a series of 25 games. After completing this requirement, I receive my assessment in the form of "Trait Report" which considers the following traits as constitutive of human behaviour: a) cognitive traits: attention, planning, flexibility, distraction, processing, memory, pattern recognition; b) emotional traits: delayed gratification, emotion, effort; c) social traits: fairness, risks, trust, learning, creativity, altruism, comfort with ambiguity. Many of these traits are divided further into sub-traits, which illustrate how one deals with different levels of risk, or is capable of reading emotion. As it says on the link "The science", these are the traits the games were designed to assess, and "were selected by the founders as (...) the traits that would be relevant for assessing career fit" (Pymetrics, 2013). If I were to agree that the above formulated traits are truly the main determining factors of my professional behaviour, I would also have to accept that I am $98 \%$ fit for a career in Business Development, 97\% fit for a career in STEM Research; 96\% fit for Investment 
Management, and barely $69 \%$ fit for a career in Writing. Based on my cognitive profile, Pymetrics matches me with an internship position for Fidelity Investment, but also recommends jobs like VP of Business Development for Imo.im, and Business Development Representative for Reonomy. My initial reaction to this assessment is emotional. I believe it enough to be flattered by the high-paying jobs presented as my best fit, and be disappointed with the lower match scores for other jobs I have believed myself well qualified for. As I remind myself that I am the critic, not the believer in algorithms, I take a further look into the details of my assessment. I find out that the one characteristic I do not have in common with writers is the fact that I am not slowed down by distractions $76 \%$ of the time. This perhaps is to suggest that writers tend to be slowed down by distractions. On the other hand, I am also deemed suitable for a STEM researcher, because I am $100 \%$ attentive. The difference in score between attention and nondistraction points to the fact that according to Pymetrics attention and distraction are not the opposite ends of the same continuum, but separate categories altogether. I open my Trait report to find out that the Distraction trait has been divided into five components: Distraction Filtering Effort, Distraction Filtering Agility, Distraction - Freedom of Distraction (Speed), Distraction Filtering Ability, Distraction - Freedom from Distraction. For each one of these I have different scores. As it turns out, I effortlessly avoid distraction $100 \%$ of the time, but I block out distractions slowly $97 \%$ of the time. Therefore, I am not slowed down by distraction $76 \%$ of the time, but I am also open to new information $65 \%$ of the time. All in all, I remain focused in a distracting environment $55 \%$ of the time. In contrast, my Attention Duration is unwavering at $100 \%$, while my Attention Control is described as $74 \%$ thoughtful rather than efficient. 
To see so many different parameters of distraction and attention matched with varied numbers is overwhelming, and makes me wonder how were these chosen as significant cognitive traits; how are they measured; what is the meaning of these scores and how do they compare to my actual life experience. As the subject of this assessment, I am initially inclined to believe it, but I then find it hard to fully agree or disagree with it for two reasons. First, I do not know what exactly these traits stand for; and second, I do not experience myself in fragments, so I don't know how I translate into a composite of pointed parameters. My behaviour in situations, my reactions to the environment depend on the meaning I ascribe to my experience, a meaning which paints a complete picture, and yet, an always changing one. How could I agree or disagree with the results with any confidence, when scenarios surging from my memory are contextually bound and both affirm and contradict these measurements.

To form an opinion on the validity of this assessment I am pressed to consider myself "objectively", that is, as an object of scientific investigation; and my behaviour as something separate and independent of me - something I have never been fully aware of or intending. Already separated from myself, 'my behaviour' or 'me' as an object of investigation, is also independent of context - it is assumed to retain its integrity beyond specific circumstances or contextual determination. This experience of the Pymetrics user is intuited in Baudrillard's description of the modern individual in one of his later books

\section{Impossible Exchange:}

In the final stages of his 'liberation' and emancipation through the networks, screens and new technologies, the modern individual becomes a fractal subject, both subdivisible to infinity and indivisible, closed on himself and doomed to endless identity. In a sense, the perfect subject, the subject without other - whose idividuation is therefore not at all 
contradictory with mass status. Quite the contrary indeed: he is the dispersal of the masseffect into each individual parcel (...).

The modern individual, being no longer part of an order greater than himself, but a victim of his own will - commanded to be what he wants and to want what he is - ends up resenting himself, and as a consequence, frittering himself away in the exhaustion of his possibilities: a new form of voluntary servitude. (Baudrillard, 2001, 47-48)

Baudrillard's premonition loses its metaphoric oomph in the context of Pymetrics and its meaning becomes very literal. The scientific or objective approach to self-investigation that the company professes entails all of the paradoxical tensions mentioned above. Users are invited to play the games for the sake of freedom - that is freedom of choice between more and better options, and freedom of better self-knowledge and control. On the other hand, for the Pymetrics tool to be necessary, the user has to agree that he does not know himself, and has to agree to be told what he wants in order to want it. It is also true that as he recognizes himself in the Trait Report, he accepts the imposition of a core "indivisible" identity, that has been derived based on likeness, rather than difference, with established models and templates, and, once assigned, will be endlessly perpetuated through networks. Virtual reality and networks, however, are too impersonal, neutral and automatic to be experienced as otherness. Hence, the Pymetrics user is automatically selfgenerated as the "subject without other", whose individuation does not contradict "massstatus", but crystallises it. He is a subject who has become the "victim of his own will" because his will as well as himself is automatically generated by the networks (i.e. Pymetrics), and his striving for 'liberation' (self-control) ironically takes the form of "voluntary servitude" (self-submission to the authority of Pymetrics).

Since I am not equipped to argue with neuroscience, I can only question the "objective" validity of my cognitive traits report by examining the structure of the 
Pymetrics games, or rather, tracing the chain of signification equivalences they base their claims on. Pymetrics games demonstrate precisely how the absolute value of the sign translates into absolute assertions of truth that, in turn, facilitate the appearance of the subject as a digital trace of itself. To illustrate this process, it is sufficient to describe not more than three out of the 20 games that are currently used to generate the "inherent" traits reports.

Let's take, for example, the game called Stop 1. This game lasts one minute. The player is instructed to press the space button every time she sees a green dot on the screen, and not to press it when she sees a red dot. Dots flash every other millisecond, changing colour in an irregular fashion. The time for response is very short. If the player manages to fulfill the instructions without a mistake she is deemed $100 \%$ Attentive, if she responds to the wrong colour, she is told that her "mind sometimes wanders" at the respective percentile. After the end of the game, a short explanation reads: "Trait: Attention Duration (+6 additional traits): We measured how you attend to the same continuous activity." For this assessment to be true, the continuous activity of pressing a button in response to a colour prompt is considered a generic sign for any type of activity that may exist, therefore the score received here is transferable everywhere else. Is this to say that if I am able to press the space bar every time I see a green dot in the span of a minute, I should also be able to listen to an hour long recitation of poetry without missing a word? If not that, what is the type of attention that is described and evaluated here, and to what kind of real-life activity does this game stylization correspond? Perhaps to account for the fact that different types of activities might require different types of attention, the pymetrics team offers an additional cognitive trait that measures attention - Attention 
Control. And although it seems reasonable to suggest that attention can be described in terms of duration as well as directionality (i.e. control), it is a bit hard to imagine how one aspect of the attention parameter can exist in the absence of the other. How can I be continuously attentive, but lose track of the direction of my attention? According to Dictionary.com the original meaning of the word "attend" comes from the Latin attendere which means literally "to stretch toward", or "stretch one's mind toward"27. And since one's mind is only applied to an object only for as long as it holds that object in mind, it seems superfluous to distinguish between attention control and attention duration. Unless, of course, neuroscience research of human cognitive processes does not require new terms to name its discoveries. If this were the case, even the definition, or the content of "attention" as a sign, is dubious, and yet, as a user, I am pressed to believe that it corresponds to something inherent in me.

Another game, where the reliance on assumed equivalences between signs and facts of life can be observed is Dots. This is a game which lasts $5 \mathrm{~min}$. The screen is divided by a X on both sides of which appear two pictures of human faces. This happens every second. The player is informed that after the faces disappear a black dot may or may not appear either on the right or the left side of the $\mathrm{X}$. The player is asked to hit the space bar every time she sees a dot appear either on the right or the left side of the X. Occasionally, one of the two faces that flash only for a few milliseconds at a time appears deformed by some strong emotion. The score is formed based on accurate response to the black dot. If the player manages to hit the space bar after every dot, she is considered $100 \%$ "emotionally steady". If she misses all dots, she is considered $100 \%$ "sensitive to

\footnotetext{
${ }^{27}$ attend. (n.d.). Online Etymology Dictionary. Retrieved August 18, 2016 from Dictionary.com website http://www.dictionary.com/browse/attend
} 
other's negative emotional states". At the end of the game, the player is informed that this game measures Emotion Sensitivity or "how likely you are to be affected by negative emotional interactions or other people's negative emotional states".

The first time I played this game, I was focused on responding to the dot. The flashing faces appeared and disappeared so quickly that I wasn't sure whether the dot comes every time after the faces, or occasionally skips. I barely noticed that some of the faces frown, and wondered what for, but only briefly. I was assessed as $64 \%$ sensitive to people's moods. The second time I played the game, I intentionally did not respond to the dot. Instead, I observed the pattern trying to figure out the reasoning behind it. I noticed that while it does alternate between the left or right side of the $\mathrm{X}$, the dot always appears after the faces disappear. Hence, the player's response is due in every single sequence of prompts. Since, I did not respond more than 5 times for 5 minutes the blurb at the end of the game read: "You tend to be sensitive to the moods of those around you. Bad moods are contagious for you, so beware!". This result unveils the underlining assumptions that make this game into a programmed test with pre-set results. Clearly, if the player does not manage to respond to the dot, she must be distracted by the frowning faces. Therefore, she is sensitive to people's emotions. Alternatively, if she is focused on the dot and responds accurately, then she is not moved by the distorted faces. Thus, lack of response to the prompt does not simply signify distraction, but distraction with the emotions on the pictured faces, i.e. "lack of response" equals "emotional sensitivity". The click of a button, or the absence of it - 1 or 0 - becomes the telling sign of the emotional characteristic of a person responsible for one's behaviour in embodied life. This is how Pymetrics translates a sign with another sign, and finds direct correspondence between the 
virtual and the real. What if that equation is not right? What if the distraction was caused by an event external to the game, or perhaps the lack of response was not even caused by distraction, but a slow reaction to the very quick prompts? Personally, I can confirm that the first time I played the game I barely noticed the faces' expressions, and yet my accuracy was only $64 \%$. I believe I was overwhelmed by the multitude of what seemed like meaningless prompts, and was trying to figure out the purpose behind them.

The enforced equivalence between different layers of signification, the sign and the real, is also well played out in the game Eyes. This is a game which lasts about 3 minutes, and is made up of 27 questions. The player is presented with 27 black and white pictures of the eyes of people from various ages and sexes, and is asked to choose the one out of four words which best describe the emotion in them. The pictures are rectangular strips which exclude the rest of the face. Each picture is shown for 6 seconds only. At the end of the game, the player is told that this game assesses the trait: Emotional Identification from Eyes. If the emotions are identified correctly, the score given is $100 \%$ "In tune with emotional cues from others' eyes", if the responses do not match any of the pre-set "correct" answers, the score given is "You are not slowed down by emotional cues" $100 \%$ of the time.

This game would function as a test under a number of presumptions. To claim that human emotions can be reliably identified from a two-dimensional photograph is to suggest that a visual sign such as the photograph can have an exact correspondence to a life emotion, and this correspondence can be trusted repeatedly. The player, however, identifies the emotion represented by the visual sign, not by experiencing the emotion, but by using a word for it - a lexical sign to translate the visual one. Then, to claim that this 
translation from visual to lexical sign is accurate means that it has somehow been verified. Perhaps, Pymetrics asked the person pictured to verify that the word chosen as the correct answer actually expresses the exact emotion that she experienced in that photo-moment. If Pymetrics researchers actually did that, they are likely to have received a very hesitant answer, because even the one experiencing the emotion would often struggle to name it with a single word. Or, were the people photographed connected to an EEG reader which traced their brain impulses and assigned specific names to each combination of signals, and then aligned it with the visual image as well? But that would be another kind of translation from electronic impulse to lexical sign to actual experience. In this sequence of translations the possibility for multiple interpretations is endless. Words cannot match emotions exactly, neither can pictures. And while the problem of language and representation has preoccupied the minds of Western thinkers for centuries, technologists like Pymetrics forego the issue of exact translation of sign to sign, and sign to reality, and assess the responses of players with enviable confidence. It is due to the absolute truth value of the sign, its direct equivalence with the real or complete indifference to it, as Baudrillard suggests, that a computer algorithm can tell me whether I am emotionally in tune with other people or not.

While the player of Pymetrics' games is constantly invited to forfeit one's inadequate subjective judgement, and accept the higher authority of algorithms, a more careful examination of the games shows that the personal trait profiles produced by Pymetrics are also the result of multi-layer subjective interpretations strung in sequences of questionable equivalences. To insist on the verification of the equivalences between these various signs, and layers of signification seems impossible, or even petty. Indeed, 
fluidity of meaning and interpretation are recognized as inseparable part of the original spoken exchange. Virtual communication, however, takes pride in verifiability, exact reproducibility of signs and clean transmission of meaning. Without consideration of the accumulating imprecision in translation, virtual images spread about as impenetrable bubbles of truth, albeit independent of any person's lived experience.

The kind of techno-scientific objectivity exemplified by Pymetrics, demands the dismantling of the person into a set of constitutional elements, which retain their meaning regardless of personal intention or variability of circumstance. Therefore, human behaviour can be detached from the human and predicted as an observable parameter. As a player, I am asked to believe that there is an objective truth about me - a core of me that is knowable, but I don't know it, so I need to be told, and I need to become, or agree that I am what I am told. By suggesting that they can predict what kind of career is most likely to make me feel happy and accomplished, Pymetrics treats even happiness as another objective parameter that is confirmable outside of subjective experience. To judge personal meaning and happiness irregardless of the person, is the kind of paradox that illustrates not only that signs are treated as proof for the existence of what they signify, but also of what Baudrillard calls the "painful revision of the principle of knowledge" (2001, p.22). As he explains in the Impossible Exchange:

Today, they say that science no longer 'discovers' its object, but 'invents' it. We should say, then, that the object, too, does more than just 'discover' us; it invents us purely and simply - it thinks us. (Baudrillard, 2001, p.23)

Accordingly, Pymetrics is the product/object of science that, in turn, invents us. 


\section{The Pymetrics' Subject in Time}

The subject as a sign of itself, is a subject "divested of its subject position" in the sense of agency or impact, but in being reduced to its "nuclear identity", it has also become infinitely and perfectly reproducible (Baudrillard, 2001, p.22, p.48). There is a solidity, and a permanence in the sign that makes it transferable from one context to the next, and this is what Derrida calls the "remainder" - the grapheme or the thing which stays the same and makes a sign recognizable from one use to the next ${ }^{28}$. While Derrida conceptualises identity as a sign, or a text, which constantly refers back to its previous contexts and anticipates its future through constant imitation of itself $^{29}$, what we see in the context of Pymetrics is not a subject constituted in language and identity emerging through the play of differences in différance ${ }^{30}$, but a subject as a virtual "remainder" - a perfectly reproducible and identical with itself grapheme. Assuming that virtual causes can produce real-life effects, Pymetrics adopts the right to claim that while they may not yet be able to program human behaviour, they can certainly predict it. Their theoretical knowledge of the inherent potential or the original blueprint of the personality of the player makes them the owner and producer of the subject as a virtual grapheme of itself.

As far as this grapheme is certain to be consistent with itself in virtual time and space,

\footnotetext{
${ }^{28}$ J. Derrida, Speech on Différance, Margins of Philosophy, 1982, electronic source: www.hydra.umn.edu/derrida/diff.html, 15 - 16

${ }^{29}$ Derrida's treatment of the subject as a text is exemplified in the work of Sidonie Smith, who sees autobiographic narration not as self-expressive but a self-forming act: "There is no essential, original, coherent autobiographical self before the moment of self-narrating. Nor is the autobiographical self expressive in the sense that it is the manifestation of an interiority that is somehow ontologically whole, seamless, and "true"'.(17) "The history of an autobiographical subject is the history of recitations of the self. But if the self does not exist prior to its recitations, then autobiographical storytelling is a recitation of a recitation." (21) Sidonie Smith, Performativity, Autobiographical Practice, Resistance a/b Auto/Biography Studies 10.1 (1995)

${ }^{30}$ Derrida describes Différance as "the movement according to which language, or any code, any system of referral in general is constituted "historically" as a weave of differences." (from J. Derrida, Speech on Différance, Margins of Philosophy , 1982, electronic source: www.hydra.umn.edu/derrida/diff.html)
} 
Pymetrics' knowledge of its characteristics in the present can be extended to the future as well. For this reason, Pymetrics' algorithms that categorise types of personalities based on cognitive parameters that are expressed in sets of behavioural characteristics and forecast respective types of performances can be deemed to constitute a specific relationship to time. This relationship to time is expressed in the use of language and practices.

Just as the game is considered a synthesis or a stylization of all life situations that call for a certain response or action, so is the person's trait report a shorthand for all possible future responses nascent in a given person at any given point of life. The value of the Pymetrics product and service is precisely its "predictive power", as advertised by the company both on its website and in the media. This is, for example, how Pymetrics describes itself in the Forbes Magazine:

Neuroscience has revolutionized our understanding of the brain and given us novel ways to assess cognitive and personality traits. We apply this neuroscience-based assessment and prediction technology to helping people find their optimal career path, and helping companies hire in a smarter and more diverse way. (Gross, 2017)

Then on their website they also state:

Resume review is both biased and lacking in predictive power. (...) Pymetrics algorithms are highly predictive and are gender, ethnic and pedigree bias free to diversify hiring. (...) Pymetrics algorithms result in a 50\% greater likelihood a hire will stay with the company. The "predictive power" Pymetrics sells is acquired by the resources of science and technology, namely the games which "assess 90 key cognitive and personality traits" that together provide a "snapshot of a person's unique characteristics". This "snapshot" is to function as a map of personal proclivities whose validity doesn't seem to expire. On the contrary, the player is allowed to play all the required games only once. Any subsequent run of the same games does not change the original score so as to preserve the 
authenticity of the assessment. As specified earlier, the user cannot "fake the game", because assessment happens at the "microsecond level, which is not a level under conscious control". In other words, my split-second reaction, of which I have no conscious control or understanding is most revealing of my 'true nature' to be expressed in present and future. Therefore, the virtual trace I have left in response to an automatic trigger, automatically prompts the printing of a label of me that should last me a lifetime (or for at least as long as it takes Pymetrics to come up with a perfected version of the games that allows and necessitates an update of users' profiles). A lifetime is condensed into a microsecond. The knowledge of me Pymetrics acquires, unhindered by my personal bias or intentions, is to be considered permanent and absolutely reliable, although I am not either permanent nor unchangeable. Moreover, this knowledge of me is received simultaneously by myself and others, and the consensus of who I am precedes any opportunity for action or becoming. I am, before I have become, and people know me before they've met me, before I have been in life.

The experience of the Pymetrics user can, thus, be summarised by Baudrillard's description of the effect of integral reality or virtuality on the subject in time:

Time itself, lived time, no longer has time to take place. The historical time of events, the psychological time of affects and passion, the subjective time of judgement and will, are all simultaneously called into question by virtual time, which is called, no doubt derisively, 'real time'. (....) With this notion of 'real time', all dimensions have contracted to a single focal point, to a fractal form of time. The differential of time having disappeared, it is the integral function that wins out: the immediate total presence of a thing to itself, which signifies that reality is henceforth the privilege of that which is identical with itself. (Baudrillard, 2005, pp.30-31)

The machinic logic of the Pymetrics algorithm is thus bound to construct the gamified subject as a predictable mechanism - a finished machine. Its algorithms produce a 
character assessment that has the quality and effect of a final judgement - a prediction that fulfils itself by laying out a program of action. This is the kind of unforgiving completeness of interpretation that Baudrillard seeks to avoid with his "theory fiction", "fables" in Cool Memories as well as most of his later writings.

As a social media product that organizes the exchange of information in the public sphere, Pymetrics' games and website constitute a cultural or a communication artefact. As such, they are a concrete manifestation of the kind of symbolic action that characterizes and constitutes the ontology of our time. Thus, the application of Baudrillard's critical prism on the analysis of Pymetrics allows me to take account of the emergence of the gamified subject as an effect of the representational structures that precede it. Baudrillard's use of "integral reality" as a descriptive label for the contemporary condition aims at the fallacies that constitute it. The equation of the sign with its referent that inserts the "code" in place of symbolic substance produces "integral reality", where the sign has been transformed into the most legitimate evidence of existence. With this new "ultra-reality" that cancels both reality and illusion, virtual experience is equated to life experience, constructed environments to natural environments, time is condensed into 'real-time' where the present and the future are equally known. Integral reality as the functioning worldview transpires in the way Pymetrics' website and games construct the real, the subject and time through discourse. Here, scientific claims are instantly validated by the mere presence of scientific terms. The game environment is treated as the condensed version of all prospective work environments, and the player's in-game performance is predictive of one's professional conduct. In other words, virtual performance is generalised into a sign for the person, 
because a person's essence can be discovered in the sum of momentous reactions to game prompts that express deep-seated inclinations. According to Pymetrics, the selfunderstanding and intentionality of the gamified subject are contingent on its selfobjectification and voluntary submission to unknown algorithms. This subject that manifests and materialises through its digital trace, has the same appeal as the virtual reality that delivers it. It is neat and complete, an intelligible mechanism that can be reliably applied or transferred from one context to the next. The gamified subject as a grapheme emerges in an ahistorical virtual moment that endows it with an air of universality and permanence. Attributes such as these may not reflect the familiar course of embodied existence, but suit better the demand for efficiency and predictability of the neo-liberal techno-scientific complex. 


\section{CHAPTER FOUR}

\section{Flow, Freedom and the Gamified Sublime}

In the preceding chapters I have traced the emergence of 'gamification' as a concept, a design strategy, and a theoretical notion. The promotional rhetoric of gamification describes it as user experience design strategy and carves a niche for its business application. The applied rhetoric of gamification sells services and inculcates users in particular subject positions and value systems. Theoretical discourse on gamification seeks to define it as a notion, situate it in a larger cultural context and evaluate its implications as a practice. In addition, my own critical analysis of gamification rhetoric and discourse centres on its ideological elements and functions by foregrounding their grammatical, rhetorical and semiotic mechanisms. Yet, in order to unravel the mysterious appeal of gamification, one also has to consider the perspective and motive of the end user. Is the gamified subject simply bedazzled into a virtual Wonderland whose enticing propositions turn out to be manipulative techniques toward ulterior ends? Or, does the deception work because it is willingly embraced?

Personal motivation has traditionally been the object of investigation of psychoanalysis, psychology and philosophy. While, I cannot presently embark on a participant-based study of gamified user experience, I choose to address the problem 
through a theoretical inquiry into the notion of "flow" which was originally coined by positive psychologist Mihaly Csikszentmihalyi and, then, used by promotional gamification rhetoric as the scientific legitimation of the power of games and gamification. 'Flow', therefore, is the conceptualisation of the subjective experience of the gamified subject, which I assess based on its relationship to several more established notions such as the sublime, freedom, and ecstasy ${ }^{31}$.

In his 1990 book Flow: The Psychology of Optimal Experience, Csikszentmihalyi describes the state of 'flow' as a moment of intense involvement when a feeling of easy mastery is combined with a dissipating sense of time and self. By examining the limits of subjective consciousness in the interplay between action and passion, intentionality and surrender, Csikszentmihalyi's concept offers ground for the conceptualization of the gamified sublime. The sublime as a liminal experience that tests and shapes the boundaries of subjectivity has its roots in its classical definitions. Edmund Burke speaks of the sublime as "delight" which "turns on pain," or a sense of "swelling and triumph" felt "when without danger we are conversant with terrible objects" (Burke, 1998, pp.8384). Similarly, Kant's discussion of the sublime as an aesthetic judgement is only part of his enduring effort to detail the functioning of the human mind. For him, it is a given that the sublime is "a disposition of the mind (...), but not the object, which is to be called sublime" (Kant, 2000, p.134). A number of contemporary theorists treat the experience of the sublime as foundational to subjectivity. Vincent Mosco claims that technological "myths that celebrate various forms of the sublime" are not simply illusions that need to be dispelled, but are meaning making and self-formulating strategies that "animate

\footnotetext{
${ }^{31}$ An earlier version of this chapter was published in 2017 under the title "The Gamified Sublime" in Trifonova, T. (ed.) Contemporary Visual Culture and the Sublime, Routledge: Taylor \& Francis Group
} 
individuals and societies by providing paths to transcendence" (Mosco, 2004, p.141, p.3). Jean-Luc Nancy emphasises that "the sublime is a feeling (...), it is the emotion of the subject at the limit" (Nancy, 1993, p.44), while Hartley points out that the problem of representation at the centre of the sublime is "the problem of the political subject's relationship to the social substance," where the subject is "nothing but the gap," the "radical split in the social-symbolic" (Hartley, 2003, p.4,p.10). Similarly, in her discussion on the digital sublime Eugenie Shinkle reminds us that aesthetic discourse itself emerged as an "inquiry into the subject"; therefore, it is less concerned with "questions of art than with the reckoning of subjective boundaries" and "definitions of subjectivity" (Shinkle, 2012, pp.94-95). Thus, the concept of the sublime does not merely refer to the qualities of the sublime object but also constitutes the relationship between an object and a perceiving subject, a relationship that is both rudimentary and extreme as the rupture of individuation. The sublime encounter, then, can be understood as the moment of separation of the one from the whole, where the imposing presence of the beyond is met by an equivalent resistance in the singular that persists regardless.

Since flow and the sublime can be both seen as formative of subjective consciousness, they are also closely associated with freedom as a dynamic relation between self and world. Csikszentmihalyi is often identified as the theoretical justification of the kind of game design that successfully produces games that are so fun that they are "addictive" but that also promote a sense of freedom and mastery. The sublime, and flow as its persuasive periphrasis in gamification discourse, point to that moment of tension between power and submission, individuation and unity. Just as Csikszentmihalyi talks about the achievement of happiness through "mastery over consciousness itself" (2008, 
p.9), Kant has postulated that "in our aesthetic judgement nature is judged [beurteilt] as sublime not insofar as it arouses fear, but rather because it calls forth our power [Kraft]" (Kant, 2000, p.145). What we see in Kant, and perhaps in Csikszentmihalyi, is the kind of freedom Jean-Luc Nancy poetically qualifies as "the sublime destination of reason itself" (Nancy, 1993, p.27). However, freedom can also be approached from the perspective of the subject's submission to the will of Providence, instinctual drives, and excess as a line of theorists starting with Edmund Burke and extending to Freud and Bataille demonstrate. This split in the understanding of freedom, or freedom as the play of diametrically opposed dispositions, is present in the notion of flow, as well as in that of the sublime.

To contextualize the promise of empowerment through game design, I align Csikszentmihalyi's theory of optimal experience with Kant's vision of the sublime as a reaffirmation of reason. To study flow as disempowerment or self-dissolution, I also read Freud and Bataille's exploration of freedom as submission to instinctual drives as the continuation of Burke's understanding of the human as directed by material forces or Providence. Such an approach is made possible not only by the origin of 'flow' in positive psychology but also by the specific relevance of the above theorists to the problems of freedom and subjectivity.

Against this theoretical background I argue that the success of 'flow' as a promotional banner for gamification consists in its meaning making capacity as a persuasive periphrasis of sublime affect. The urge towards a theoretical explanation of the mysterious power of video games is also an indication of the felt need among designers of virtual reality to endorse the virtual experience not only as an event capable of producing personal fulfilment and meaning, but also as important to the formation and expansion of 
subjective consciousness. In this sense, the concept of 'flow' as used by game designers and gamifiers hints at the possibility of designing and utilizing the sublime, that is, at the equivalence of the virtual and the real. In addition, by adopting the notion of 'flow' as its causa sui, gamification justifies its ambition to coordinate the diverse interests of designers, users, and corporate clients by attempting a solution to the long standing challenge of political economy to reconcile the free willed subject with the well-utilized one.

\section{The Sublime Effects of 'Flow'}

The experience of flow as formulated by Csikszentmihalyi and re-interpreted by gamifiers, can be seen to describe the impact of the sublime encounter as a movement from the overpowering experience of something greater than oneself to an empowering sense of being in control. This movement is clearly distinguishable in the writing of gamification theorists and promoters, who use the notion of flow to justify the capacity of games to simultaneously empower and overpower the gamified subject.

As one of the pioneers and ideologues of gamification, Jane McGonigal sees the transformation of trivial activities into games as an opportunity for a more positive relationship to the world that promotes both individual growth and social advancement. On the one hand, she emphasises the personal agency of players by stating that "we have to make our own happiness" by engaging in "autotelic" ${ }^{32}$ activities that provide "intrinsic" rather than "extrinsic" rewards. On the other hand, she also boasts that "game developers know better than anyone else how to inspire extreme effort and reward hard work"

\footnotetext{
${ }^{32}$ Jane McGonigal describes games as "the quintessential autotelic activity" which is freely chosen and rewarding in itself (McGonigal 2011,2)
} 
(McGonigal, 2011, p.13). Therefore, games are "a unique way of structuring experience" that can generate "extreme emotional activation," or inspire and motivate "millions of people at a time" (McGonigal, 2011, pp.28-34). As the power of the player appears to be framed and preconditioned by the power of the game, McGonigal seeks support from Csikszentmihalyi whom she credits with founding 'the science of happiness'. His theory of optimal experience explains how, when freely entered, the structure of "games and gamelike activities" presents the necessary conditions for the experience of the "intense, joyous engagement" he has called 'flow' (McGonigal, 2011, p.35). McGonigal also points out that game designers - "the most talented and powerful happiness engineers on the planet"-are specifically trained to "relentlessly pursue happiness outcomes, including flow," and their expertise should be employed in the organization of daily life (McGonigal, 2011, p.38).

Tom Chatfield, another proponent of gamification or serious games, discusses the mysterious attraction of fun and games and how these can be applied in "the realms of business and public service" (Chatfield, 2010, p.153). Similar to McGonigal, he argues that a world designed according to the principles of play will be a more ethically elevated and friendlier place. However, he still treats games as means towards ulterior ends, and the satisfaction of the player as the necessary condition for their efficacy: "If the future is looking more and more like a game, it's because the science of satisfaction has never before been so precise, so powerful, or so profitable. Where play goes, the world will follow" (Chatfield, 2010, p.38). The 'science of satisfaction' Chatfield is referring to is again Csikszentmihalyi's theory of optimal experience. Based on it, the utilization of play becomes possible by means of isolating flow as the principle of fun and then applying it 
to other contexts. The conditions Csikszentmihalyi outlines for reaching this mental state are interpreted as a recipe that calls for the right "balance of rules, actions and consequences" that create "ceaseless demands on our attention" (Chatfield, 2010, 42-43).

If McGonigal and Chatfield are concerned with the humanistic promise of a gamelike world, writers like Zichermann and Hugos are more interested in promoting games as tools for engagement with considerable business potential. Hugos' book Enterprise Games clearly treats the concept of flow as a prescription of how to build "engagement engines" (Hugos, 2012, p.2). To work reliably these should be designed with four features in mind: "goals, rules, feedback systems, and voluntary participation" (Hugos, 2012, p.12). Then, he clarifies that the right balance of the first three is "what induces voluntary participation" (Hugos, 2012, p.12). Zichermann, the author of Gamification by Design, directly states that gamification is a way to "get people to take actions that they don't always know they want to take" (Zichermann, 2011, p.15). He is also quick to admit that it is the idea of flow that is "at the heart of the success of games" (Zichermann, 2011, p.16). Zichermann's treatment of the player may be patronizing, but the more careful language of the rest of the authors still caries a similar message. Unlike the traditional reward system, which caters to material needs, gamification seeks to take control over personal motivation by targeting desire and meaning making processes. However, the gamified subject's 'deep' satisfaction is only an interim goal, a stepping-stone to an even greater purpose. Like games themselves, the player is ascribed agency and power in order to be transformed into effective means. Thus, flow, which serves to explain the capacity of games to subordinate by means of exhilaration, appears to produce the sublime affect, but in reverse. 
Without the inkling of a mysterious power that lures us despite our intentions, games and gamified environments would be no different from other organizational structures. Hence, the notion of flow is employed by gamifiers to bridge the gap between mystery and instrumentality, spontaneity and predictability, doing for doing's sake and utility. Still the question remains: what is it about the state of flow that supports both the possibility for autonomous, self-motivated action, and the subject's vulnerability to external manipulation? To answer this, I look at Csikszentmihalyi's own definition of the notion. I examine the empowering effect of flow as a method of structuring the contents of consciousness in the context of Kant's understanding of the sublime as an experience that "awakens the feeling of a supersensible faculty in us" (Kant, 2000, p.134). Then, I study the overpowering effect of flow as a loss of self-consciousness in view of Freud and Bataille's treatment of freedom as submission to instinctual desires.

\section{Flow as Being in Control}

In his Analytic of the Sublime Kant explains that the feeling of the sublime "is a pleasure that arises only indirectly, being generated, namely, by the feeling of a momentary inhibition of the vital powers and the immediately following and all the more powerful outpouring of them" (Kant, 2000, p.129). His description of restraint followed by a release calls to mind a moment of shock and exhilaration when one holds one's breath before letting out a scream of excitement. Or perhaps, the "momentary inhibition of vital powers" refers to a physical or emotional exertion, which mobilizes the whole being into a strenuous suspense. Csikszentmihalyi's description of optimal experience or flow approaches closely this second interpretation. He explains that optimal experience occurs 
“when a person's body or mind is stretched to its limits in a voluntary effort to accomplish something difficult and worthwhile" (Csikszentmihalyi, 2008, p.3). The experience of flow, he adds, is usually earned through pain, persistent effort and discipline, while the pleasure we derive from it comes from the "sense of mastery-or (...) participation in determining the content of life" (Csikszentmihalyi, 2008, p.4). It appears, then, that the state of flow, like sublime affect, is about the collision of pleasure and pain, mastery and deference, that overflow into one another. This is the psychological drama of the subject at its limit, who witnesses and partakes, submits to but also initiates the formational exchange between self and world. However, while Kant speaks of the sublime as an encounter that has to be incorporated into consciousness through a "supersensible" faculty, Csikszentmihalyi develops a technique for "getting control of life" through "achieved control over psychic energy" and "consciousness itself" (2008, pp.6-9). From this perspective, flow is not only consciousness expanding but can be reinterpreted as a kind of intentionally sought sublime affect.

In contrast to classical Freudian psychoanalysis, which is based on the study of pathologies, Csikszentmihalyi collects his data about the experience of 'flow' from people who describe their happiest moments. According to his respondents, these are moments "in which people are so involved in an activity that nothing else seems to matter" (Csikszentmihalyi, 2008, p.4). Distinguishing between pleasure and enjoyment in a way reminiscent of Kant's distinction between the experience of beauty and the sublime, Csikszentmihalyi claims that "enjoyment happens only as a result of unusual investment of attention" or conscious effort (Csikszentmihalyi, 2008, p.46). Therefore, extreme sports make some of the most effective flow inducing activities, where enjoyment, the 
author stresses, is not derived from "courting disaster," but from "the perfectly healthy feeling of being able to control potentially dangerous forces" (Csikszentmihalyi, 2008, p.60). Thus, Csikszentmihalyi conceptualises optimal experience as pleasure earned through pain, or as the result of the imposition of a structure, which organizes the contents of consciousness in order to produce the unobstructed flow of psychic energy ${ }^{33} 34$.

Whether a person creates her own structure or enters an already existing one the organization of psychic energy into flow is achieved only by a willing and wilful participant. As Csikszentmihalyi explains, the produced system of action "takes its form from the rules of the activity; its energy comes from the person's attention" (2008, p.65). In other words, flow is possible when an activity is embraced, based on a subjective criterion, as "autotelic" — enjoyable for its own sake, rather than as means to an end. Thus, the sense of "exercising control in difficult situations" comes as a result of the voluntary

${ }^{33}$ Csikszentmihalyi is admittedly influenced by the American philosopher and psychologist William James (1842-1910) who is the first to claim that consciousness resembles a "stream" of stimuli rather than a linear succession of "ideas." Moreover, the human capacity for attention "to one thing rather than another is for James the sign of an "active element in all consciousness,... a spiritual something... which seems to go out to meet these qualities and contents, whilst they seem to come in to be received by it"". In other words, according to James the free will of human beings consists primarily in their capacity to direct their own attention. [Online Source]: Goodman, Russell, "William James", The Stanford Encyclopedia of Philosophy (Summer 2017 Edition), Edward N. Zalta (ed.), [Available at: <https://plato.stanford.edu/archives/sum2017/entries/james/>] Goodman referring to James, W. The Principles of Psychology, Cambridge, MA: Harvard University Press, 1981. Originally published in 1890 [p285].

34 Csikszentmihalyi's concept of flow is also reminiscent of John Dewey's description of "experience" vs. "an experience". Indiscriminate, humdrum "experience" lacks the "interest that controls attentive rejection or selection of what shall be organized into the developing experience". Impressions and occurrences stream towards us, "but they are neither definitely included nor decisively excluded; we drift". On the other hand, "an experience" is formed when "the material experienced runs its course to fulfillment". As Dewey further writes: "In such experiences every successive part flows freely, without seam and without unfilled blanks, into what ensues. At the same time there is no sacrifice of the self-identity of the parts. (...) In an experience, flow is from something to something. As one part leads into another and as one part carries on what went before, each gains distinctness in itself. The enduring whole is diversified by successive phases that are emphases of its varied colors." (Dewey, J. (1939) Art as Experience. New York: Capricorn Books [pp. 35-57].) [online] Available at: https://www.marxists.org/reference/subject/philosophy/works/us/an-experience.htm [Accessed 8 July, 2017]. 
participation of an autonomous actor, who chooses to attach value to specific goals and challenges. So defined, the state of flow closely approximates modern concepts like intentionality and free will. Remarkably, however, the experience of control and mastery in flow coincides with a dissipating sense of self. This momentary freedom from selfconsideration and signification is not only enjoyable, but allows the experience of unity with something other. As the actor becomes one with the action, "what slips below the threshold of awareness is the concept of self" (Csikszentmihalyi, 2008, p.64). However, as Csikszentmihalyi explains, this "loss of self-consciousness can lead to selftranscendence, to a feeling that the boundaries of our being have been pushed forward" (2008, p.64). And though he recognizes that this may seem paradoxical, he also claims that "giving up self-consciousness is necessary for building a strong self-concept" (Csikszentmihalyi, 2008, p.65).

In his own description of the nature and conditions of optimal experience Csikszentmihalyi describes the complex notion of flow as delineated by a constant movement between extremes. The unobstructed movement of psychic energy is ensured by attention structuring mechanisms. Flow is willed by the participant in the activity, yet it can be addictive. The experience of autonomy and mastery over an object or an action is emphasized by a feeling of unity and an absent sense of self. Such fluctuations between order and disorder, control and surrender, autonomy and dependence, describe the complex ways in which the self is negotiated through its relationship to an outside world.

Csikszentmihalyi's awareness of the implicit tensions on which the concept of flow rests shows at various points in his work. According to his own model of the self "the most basic fact about persons is that they are not only aware of their own existence 
but can assume control of that existence" (Csikszentmihalyi, 1981, p.2). After taking "self-awareness and self-control as givens," Csikszentmihalyi and Halton explain "selfawareness occurs when the self becomes the object of reflection — that is the self takes itself as its own object" (1981, p.3). In view of his own definition of the self, Csikszentmihalyi has to admit that there is something "paradoxical [in the] relationship between losing the sense of self in a flow experience, and having it emerge stronger afterward" (2008, p.65), yet he continues to maintain that retaining self-consciousness in a state of flow indicates a lesser degree of involvement ${ }^{35}$. Such a claim may resonate with the reader's own experience but constitutes a contradiction in terms according to Western concepts of subjectivity. As Brinkmann points out, the defining feature of modern subjectivity is precisely the idea that "object- and self-awareness mutually imply one another", which also makes "self-referentiality a necessary condition of intentionality or object-directedness" (Brinkmann, 2005, p.33). If one's relationship to objects in the world is preconditioned by self-awareness, how can we conceive of 'flow'?

Csikszentmihalyi's alternation between emphasizing the need for control over one's consciousness and insisting that self-consciousness itself dissipates in the exact moment of controlled action calls to mind Kant's statement that "the mind feels itself moved in the representation of the sublime" (Kant, 2000, p.141). Kant compares this movement to a "vibration" between a "repulsion" and an "attraction": the first caused by

\footnotetext{
${ }^{35}$ Csikszentmihalyi explains that "preoccupation with the self" is a protective measure which causes the expenditure of great amounts of psychic energy as it seeks to restore functional order to consciousness. In this regard, he claims, it is not surprising that the absence of self-awareness which becomes possible in the absence of fear or self-concern, is usually experienced as freedom to act. Conversely, he also writes: "It almost seems that occasionally giving up self-consciousness is necessary for building a strong selfconcept. (...) In flow a person is challenged to do her best, and must constantly improve her skills. At the time, she doesn't have the opportunity to reflect on what this means in terms of the self - if she did allow herself to become self-conscious, the experience could not have been very deep. But afterward, when the experience is over and self-consciousness has a chance to resume the self that the person reflects upon (...) is now enriched by new skills and fresh achievements." (Csikszentmihalyi, 2008, 65-68)
} 
"the inadequacy of the imagination" stretched to its limits before "an abyss, in which it fears to loose itself"; and the second caused by the fact that "the subject's own incapacity [Unvermögen] reveals the consciousness of an unlimited capacity [Vermögen]" (Kant, 2000, pp.141-142). This vibration or flow can also be understood as the transition from representational-conceptual thinking to aesthetic perception. In other words, the immersion into sensual input is experienced as a momentary detachment from processes of conceptualization, which resume shortly after to absorb and distribute the new stimuli into the existing scheme of meaning. Kant refers to the same movement from sensual to sensible perception in his definition of the sublime: "That is sublime which even to be able to think demonstrates a faculty of the mind that surpasses every measure of the senses" (Kant, 2000, p.134). Consciousness, overwhelmed by the chaos of sensual stimuli, is about to lose itself in the abyss, but instead remains intact and integrated in the face of it.

The tension between empowerment and disempowerment characterises both the concept of flow and that of the sublime. However, for Kant and Csikszentmihalyi the abyss of the unknown, or the immersion into indiscriminate sensual perception, is that which calls the subject to an awareness of the mind's power to organise and structure reality. By emphasising the consciousness expanding function of flow, Csikszentmihalyi restores Kant's faith in the "unlimited capacity" of reason. However, what distinguishes him from Kant is the claim that optimal experience can be intended and planned for. His idea of flow as an instrument for self-regulating the contents of one's consciousness is precisely what appeals to gamifiers. The problem is that this leaves us with a selfgenerating and self-regulating subject without an exterior. 
Alternatively, the problem of personal freedom (or empowerment and disempowerment) can also be approached from the perspective of Edmund Burke, Freud and Bataille who consider the body and instinctual drives as the original cause of psychic activity.

\section{Flow as Self-Preservation}

In as much as the sublime can be conceived as the traumatic but formational process of withdrawal of the self from the world, it seems appropriate to extend the exploration of the gamified sublime to Freud's conceptualization of liberty as the negotiation between the life and death drives, and Bataille's discussion of "sovereignty" as the tension between singularity and unity, social conformity and excess. As a continuation of Burke's physical sublime, Freud and Bataille offer an important counterbalance to the modernist precedence of reason instituted by Kant and still resonating in Csikszentmihalyi's presentday positive psychology. Ironically also, the discussion of freedom from the perspective of the liberated body and instinctual desire implies the submission of reason to sensual input or material forces beyond its control. In flow, the precedence of the body is marked by the dissolution of self-consciousness in the moment of intense physical engagement ${ }^{36}$. The idea of the primary role of body and senses in the organization of human reality goes back to Burke's discussion of the sublime in A Philosophical Enquiry into the Origin of Our Ideas of the Sublime and Beautiful. His classification of human passions already anticipates what Freud would later call the life and death drives ${ }^{37}$ :

\footnotetext{
${ }^{36}$ Check footnote 2 .

${ }^{37}$ As I explain in the next section, Freud defines the death drive as the aggressive ego-instinct "which $\operatorname{aim}(\mathrm{s})$ at preserving the individual", hence he also compares it to hunger or necessity [Ananke]. The life
} 
Most of the ideas which are capable of making a powerful impression on the human mind, whether simply of Pain or Pleasure, or of the modification of those, may be reduced very nearly to these two heads, self-preservation and society; to the ends of one or the other of which all our passions are calculated to answer. The passions which concern selfpreservation, turn mostly on pain or danger (...), and they are the most powerful of all the passions. (...)

The other head under which I class our passions, is that of society, which may be divided into two sorts. The society of the sexes, which answers the purposes of propagation; and next, that more general society, which we have with men and with other animals, and which we may in some sort be said to have even with the inanimate world. The passions belonging to the preservation of the individual, turn wholly on pain and danger: those which belong to generation, have their origin in gratifications and pleasures. (Burke, 1998, pp.57-61)

Whether Freud's life and death drives ("Eros" and "Thanatos") are directly borrowed from Burke or not, the close parallel between their ideas points to a long line of thought that gives precedence to nature or Providence as a regulating principle, and to the body as their instrument. Burke's definition of the sublime is embedded in his understanding of the human constitution as that "which Providence has framed in such a manner as to find either pleasure or delight (...) in whatever regards the purposes of our being” (Burke 1998, 80). The sublime, according to Burke, can "excite" a myriad of "passions" such as "delight," "astonishment," "awe," but all of these are founded on a peculiar mixture of pleasure and pain. Delight is pleasure that "turns on pain," and astonishment is "that state of the soul, in which all its motions are suspended with some degree of horror" (Burke, 1998, p.85, p.94). Hence, the "idea of pain and danger," which is implied in the experience of the sublime affiliates it with the urge for "self-preservation." However, the actual absence of "such (directly threatening) circumstances" also gives us pleasure, or

drive or Eros, on the other hand, refer to the object-instincts, whose function is the preservation of the species (Freud 1961, 76). 
else, affiliates the sublime with the passions under the head of "society." Translated into Freudian terms, Burke's definition treats the sublime as a liminal state that draws on the energies of both Eros and Thanatos. It is the experience of the ego confronted with the fear of death or dissolution into otherness. Yet, the presence of the "terrible," at an arm's length, also reactivates or draws the ego's boundaries, making them palpable and vibrant. Thus, Burke's understanding of the sublime prepares the ground for the conception of freedom as submission to the body and its demands for pleasure.

At the level of the body and instinct the movement between being in control and resignation is still present, but it has been redressed. Since pleasure and pain are first bodily sensations and then psychological principles, the sublime appears as the unavoidable pain of being, as absolute otherness encountered in the face of another person or society. Therefore, rather than speaking about battling faculties of the rational mind, the presence and absence of consciousness, Freud talks about the struggle of conflicting desires. For him the problem of freedom is the problem of the origin of the autonomous self whose needs are both provided for and frustrated by society.

In Civilization and its Discontents Freud situates the conflict between individual liberty and society at the very origin of the ego. According to him, the unitary sense of self emerges as a self-protection mechanism. The distinction between inner and outer stimuli, which is not yet present in the primary ego-feeling of the infant, is motivated by the need to separate and protect the ego from outside sources of pain and displeasure. As Freud explains, the infant who feels one with the external world "separates off an external world from itself" (Freud, 1961, p.15). From here on, the ego's pursuit of pleasure is regulated by the reality principle, which protects it from frustration by coordinating its 
claims with external reality. Further on, pointing at the object-directedness of the self, Freud explains the origin of civilized life as follows: "The communal life of human beings had, therefore, a two-fold foundation: the compulsion to work, which was created by external necessity, and the power of love, which made man unwilling to be deprived of his sexual object (...). Eros and Ananke [Love and Necessity] have become the parents of human civilization too" (Freud, 1961, p.55). Love (as object-directedness) and necessity (as ego-directedness) or "hunger and love", as he quotes Schiller later, are human needs which civilization is meant to satisfy, but which also reflect man's natural constitution. 'Hunger' for Freud signifies the aggressive ego-instincts "which aim at preserving the individual" (death drive), while 'love' indicates the object-instincts, whose function is the preservation of the species (Eros) (Freud, 1961, p.76).

The familiar conflict between individual and civilization, or self and other does not simply result from the opposing interests of these two drives. The urge to aggression does not always protect the individual, neither does Eros always serve the purposes of the species. Both the instincts of life and death can have an inward or outward orientation. The problem arises from "within the economics of the libido" (Freud, 1961, p.106), which has to be distributed between the ego and its objects. As Freud explains, "any restriction of this aggressiveness directed outwards would be bound to increase the self-destruction" (Freud, 1961, p.78). Conversely, the love of the other, takes away from the love of the self. In this system, Eros and Thanatos are the two extremes of the same movement judged from the perspective of libidinal investment. The same principle underlies the function of the super-ego, which demands instinctual renunciation out of "fear of loss of love" (Freud, 1961, p.85). The aggression of the super-ego towards the ego, Freud 
suggests, returns in equal measure the original aggression of the ego's desire for an external object. In other words, the greater the desire for the other, the greater the fear of loss of love, the greater the self-imposed renunciation.

Clearly, the liberty to indulge one's instinctual desires is frustrated by inner discord, rather than external imposition. The ego's claim to autonomy appears as the defensive reaction of a spoiled child who has been denied the satisfaction of his whims and now stomps its feet in defiance. Otherness is hurtful, hence it is expelled; but otherness is also the source of pleasure that has to be regained. Phrased differently, the sublime encounter translated into the drama of emerging self-consciousness teaches that freedom is a dynamic exchange between self and world. It is a form of breathing where restraint is necessarily followed by release, and where being in control is preconditioned by surrender. Having started with the capacity of humans to build and structure the contents of consciousness, Csikszentmihalyi reaches the peak of optimal experience to find a kind of merging of self with action, and a channelling of energy that is more akin to release and surrender than to control. Freud, on the other hand, understands human nature as driven by instincts and aggression, only to observe how the spontaneous pursuit of pleasure transforms into instinctual renunciation and sublimation under the threat of loss of love.

Thus, if structured, flow-inducing activities are seen as a product of civilization, and are treated as the battle ground of Eros and Thanatos, it becomes easier to understand how they can be both empowering and subordinating. The blending of a sense of mastery with a sense of self-dissolution in the state of flow parallels the change in significance of the libidinal investment, which shifts from Eros to death drive depending on direction. In 
view of Freud's ideas, the "joyous self-forgetful involvement" that allows

Csikszentmihalyi's subjects to feel at one with the world can be read as a return to primitive narcissism. At that early moment of psychic development the ego is still not clearly demarcated and is dominated by a feeling of "limitlessness and of a bond with the universe" (Freud, 1961, p.15). On the other hand, if the "oceanic" feeling, which Freud also considers the basis of religious thinking, turns out to be present in the mature individual, it might as well be equated with Csikszentmihalyi's understanding of flow. Based on its presently expanded definition, flow is the state in which psychic or libidinal energy is so channelled that life and death drives are, at least momentarily, harmonized to serve the interests of both the individual and the species. The non-distinction between 'the inner' and 'the outer' in that very moment is a kind of freedom founded, simultaneously, upon mastery and submission, because the autonomy of the self is hardly more important than the possibility of its transcendence.

\section{Bataille on Eroticism and Sovereignty}

Bataille is another author seeking a standpoint for the exploration of human nature from which the coordination of the divergent "potentialities of the human spirit" would be possible. In Death and Sensuality he brings Freud's notions of Eros and Thanatos to their logical completion. Rather than upholding Freud's vision of a human torn apart by irreconcilable life and death instincts, Bataille seeks the "fundamental unity of the human spirit"(2006, p.1). Eroticism, according to him, is precisely that place where life and death intersect. This is not simply to say that the life and death drives coincide, rather to propose that "at the deepest level" perceivably irreconcilable and "extreme human 
potentialities" express the same aspiration. "Beyond their mutual exclusiveness", religious experience and erotic impulse, divine ecstasy and extreme horror reach out towards the sacred. As Bataille sums it up in his foreword, "human passion has only one object in this forlorn world of ours", which is most commonly seen in "the image of God". With this statement, Bataille ties together the sublime as the search for selftranscendence with the sublime as the generation of self-consciousness, and exposes them as dynamic reverberations of the same formational encounter.

Eroticism as the founding principle of the human spirit is played out in every aspect of life. Similar to Freud, Bataille describes the erotic nature of the relationship between self and community, and mechanisms of individuation and socialization. If eroticism is the human way of being and interacting with the world, that is because it is an original aspect of inner experience. As Bataille points out, "man is everlastingly in search of an object outside of himself, but that object answers the innerness of the desire" (2006, p.29). Eroticism is most readily understood at the physical level where "reproduction implies the existence of discontinuous beings" (2006, p.12). Each singular, finite being which is born alone and dies alone, also gives birth to the next one. In that sense, Bataille claims, the "gulf" between us is death, but it is also the "continuity" of being. As the human modus operandi, eroticism then is the magnetic tension which both enables the withstanding of distinct beings from one another, and at the same time responds to their inner need to break through the enclosures of singularity. As Bataille writes:

We are discontinuous beings (...) but we yearn for our lost continuity. We find the state of affairs that binds us to our random arid ephemeral individuality hard to bear. Along with our tormenting desire that this evanescent' thing should last, there stands our obsession with a primal continuity linking us with everything there is (Bataille, 2006, p.15). 
Bataille's indebtedness to Freud is noticeable here. The life drive, which protects the ego's interest and independence, finds expression in the persevering idea of individuality. The death drive, the desire for the other, coincides with the yearning for continuity, which can reconstruct the "oceanic" feeling of being one with "everything there is". The important distinction, however, is that while for Freud the work of Eros and Thanatos translates into a conflict between ego and civilization, self and social directedness, Bataille's eroticism absorbs these duelling tendencies and brings the tension even closer to the center of the self. Rather than an ego poised against its exteriority, now we have an ego folded onto itself. For that reason, while considering eroticism "an immediate aspect of inner experience" (2006, p.29), Bataille also sees it as the urge to exceed the confines of the discontinuous self:

I said that I regarded eroticism as the disequilibrium in which the being consciously calls his own existence in question. In one sense, the being loses himself deliberately, but then the subject is identified with the object loosing his identity. If necessary I can say in eroticism: I am loosing myself.(Bataille, 2006, p.31)

Bataille's affirmation that erotic desire "presupposes a partial dissolution of the person" or the destruction of "the self-contained characters of the participators" (2006, p.17), corresponds closely with Csikszentmihalyi's description of the effects of flow. The "dissolution" of the self both authors speak about is not a superficial coincidence of terms. Similar to the state of flow, which paradoxically dissolves self-consciousness into pure action, eroticism dismantles the integrity of the discontinuous being to open it up towards continuity. This movement between self-possession and self-abandonment observed both in flow and eroticism offers an important insight about the nature of the relationship between self and world. Conventional understanding of freedom seems to 
account for only a part of this relationship. It considers it from the perspective of an insatiable, but defensive master self, whose agency is contingent on the intact presence of the agent. For Bataille, as well as Csikszentmihalyi, the so called "dissolution" or dispossession of the self is just as desirable as the sense of mastery. Rather than a disappearance, Csikszentmihalyi sees the momentary lapse in self-awareness as an outbound redirection of one's energy and attention. For Bataille on the other hand, if there is a path to ultimate self-control, it runs precisely through surrender to excess and violence.

To observe how each tendency when pushed to its extreme inevitably morphs into its opposite is Bataille's main goal in Death and Sensuality. He delves into a variety of social contexts to demonstrate that "the urge towards love (...) is an urge towards death" (2006, p.42); taboos give shape and meaning to transgression rather than eliminating it; the sacred "owes its character to the profane world it denies" (2006, p.115). It is the nature of eroticism to both contain and reinvigorate the disparity between opposites so as to ensure its own perpetuity. This is precisely what validates it as a founding principle of interaction in a homocentric psychic universe.

By the same token, one can approach the paradox of personal freedom and begin to understand how self-affirmation verges on self-denial, and the highest form of control is a kind of wilful surrender. The movement that sustains such dualities is traced in Bataille's discussion of Marquis De Sade's sovereign man. He uses the composite image of De Sade's gathering of sadistic characters as a way to envision the logic of transgression at its last extreme. In resonance with Freud's theory of primary drives, the sovereign man which emerges from De Sade's works is the literary epitome of liberty as 
the uninhibited pursuit of pleasure ${ }^{38}$. As Bataille explains, pleasure - an end in itself is “essentially extravagant", excessive, tending toward "ruinous waste", degradation and death. In their unrelenting quest for intense pleasure De Sade's protagonists cannot afford to be softened by sympathy or solidarity with others. Sovereignty reigns in absolute solitude, because one "who admits the value of other people necessarily imposes limits upon himself" (2006, p.171). Hence, Bataille continues, the cost of "unfettered freedom" is "a sort of heroic cynicism", an "apathy" which requires extraordinary discipline and strength to be sustained. The consistent opposition to affection, passion and spontaneity is needed for the upholding of sovereignty, but runs counter the initial strife for pleasure. Ironically, Bataille observes, “all the great libertines who live only for pleasure are great only because they have destroyed in themselves all their capacity for pleasure" (2006, p.173). Traced to its logical conclusion De Sade's treatment of sovereignty reveals that "at the very peak of unlimited denial of others is a denial of oneself" (2006, p.174). Thus, what has initially began as an "attitude of utter irresponsibility", has become "stringent self-control" (2006, p175); or else, violent excess has adopted the calm strategies of reason.

To bring the provocation to completion, Bataille also aligns eroticism with mystical experience. He claims that the sovereignty achieved through desensitization from excess is of the same quality as sovereignty reached through wilful abstinence and

\footnotetext{
${ }^{38}$ Baudrillard's critique of Marxism in The Mirror of Production and in Symbolic Exchange and Death is heavily influenced by Bataille's ideas of pleasure and excess as expressions of human liberty and as the natural antidotes to capitalist productivity. As Douglas Kellner points out, Baudrillard's "term "symbolic exchange" was derived from Georges Bataille's notion of a "general economy" where expenditure, waste, sacrifice, and destruction were claimed to be more fundamental to human life than economies of production and utility". (Kellner, Douglas, "Jean Baudrillard", The Stanford Encyclopedia of Philosophy (Winter 2015 Edition), Edward N. Zalta (ed.), URL = $<$ https://plato.stanford.edu/archives/win2015/entries/baudrillard/>.)
} 
asceticism. Both of these entail "non-attachment to ordinary life, indifference to its needs, anguish felt in the midst of this until the being reels, and the way left open to a spontaneous surge of life that is usually kept under control but which bursts forth in freedom and infinite bliss" (Bataille, 2006, p.247). What Bataille describes here is the process of breaking through the boundaries of the discontinuous being in order to access a beyond. Whether one would choose violent excess, or stringent discipline as a starting point, the urge toward continuity requires unwavering commitment, and resignation to the possibility of pain. These, can then procure "the colossal concentration of energy" (2006, p.174) needed to reach a point of spontaneous release and ecstasy. Religious eroticism, as he calls it in the beginning of the book, is only one of the three types of eroticism (physical, emotional, and religious), but the one which most directly points to its ultimate purpose in the pursuit of the sacred. In the moment of theopathic elevation, Bataille explains:

(...) the subject becomes passive and suffers what happens to him with a kind of immobility. (...)The object of contemplation becomes equal to nothing, and at the same time equal to the subject. There is no longer any difference between one thing and another in any respect; (...) the subject lost in the indistinct and illimitable presence of the universe and himself ceases to belong to the passing of time. (Bataille, 2006, p.249)

The theopathic state, which Bataille considers the final destination on the road to "complete sovereignty" is reminiscent of Kant and Burke's definitions of the sublime as a burst or "outpouring" following an "inhibition", and, by the same token, is evocative of Csikszentmihalyi's concept of flow. Sovereignty and flow are thus achieved through a kind of controlled loss of control that is the encounter with the sublime. The subject Bataille describes is not active, but receiving, not autonomous, but one with the universe. Likewise, Csikszentmihalyi who draws a parallel between advanced meditation and flow, 
points out that samadhi is the highest "stage of "self-collectedness", when the meditator and the object of meditation become as one" (2008, p.105). Aware of the conflict between means (strict discipline of attention), and end (the dissolution of the self into unity without discrimination or bounds), Csikszentmihalyi feels the need to further affirm their relation. Hence, he explains that:

(...) till the final stage of liberation, the yogi must maintain control over consciousness. He could not surrender his self unless he was, even at the very moment of surrender, in complete control of it. Giving up the self with its instincts, habits, and desires is so unnatural an act that only someone supremely in control can accomplish it (Csikszentmihalyi, 2008, p.105).

Flow, then, is "liberation" reached through controlled surrender. Like eroticism, it opens every extreme towards its alternative, running like electric current between opposite charges.

It is interesting to observe how thinkers who begin their investigations into human nature from diametrically opposed departure points, finally arrive at the same conclusions. While Csikszentmihalyi starts with the assumption that humans are capable of directing their attention and organizing consciousness in productive ways, he is also pressed to recognize that crime, addictions and alcoholism are also flow-producing mechanisms. Freud and Bataille, on the other hand, having started with the belief that excessive self-indulgence is truer to human nature than law-abiding discipline, come to recognize that the kernel of stringent self-control is also found in the unrestrained pursuit of pleasure. Freud's approach to this paradox is to insist on the opposition of the two primary drives. In his framework, the ego's urge to pleasure is part of the individuation process. The death drive, associated with the ego's aggression, hunger and consumption is 
self-sustaining but implies death for the other. Eros, on the other hand, as the urge towards union with others is altruistic. It supports the other, the unity, the good of all, but not the individual self. It is Eros that restricts instinctual desires, demands self-inhibition or the death of the individual who is now merged with, fed into the whole. Freud's enforcement of a strict opposition between the life and death drives seems too rigid to Bataille. He considers the two primary drives in parallel and rightfully observes that while, both conclude in death, they originate as life's sustenance. As far as the urge to pleasure consumes both self and other/ness, it does not need to be distinguished from the urge to union as self-destruction. Conversely, Eros is not altruistic, but merely strategic, because its original motivation also is pleasure through the other. Here comes to mind the very opening remark of Death and Sensuality: "Eroticism (...) is assenting to life up to the point of death" (Bataille, 2006, p.11). In other words, life is death and death is life because what sustains life also exhausts it. The paragon of bliss is the infantile regression to harmonious unity with the entirety of the universe which breathes in resonance with our innermost desires. Flow, the "oceanic" feeling or ecstasy offer but a momentary glimpse into this utopia that cancels the beyond together with the self for the sake of oneness. Then, we necessarily return to difference, which is life.

\section{Flow as the Gamified Sublime}

As Nancy has asserted, the sublime is the experience of the subject at the limit. Whether it tests the limits of consciousness, comprehension, imagination or the senses, the sublime is that interruption, that flood of world towards the unitary self that threatens its integrity. For Kant the sublime constitutes an abrupt encounter with something radically different 
and greater than the subject, whose senses are intensely overwhelmed, but whose faculty of reason regains control over meaning making processes. His ideas are the foundation of Csikszentmihalyi's insistence that attention, psychic energy and the contents of consciousness can be directed by the will of a subject capable of control over his existence. However, optimal experience is not simply consciousness expanding. It is a reach beyond the self, a momentary contact with otherness that can be intentionally sought after.

At the other end of the spectrum, Edmund Burke also speaks of "terror" as the ruling principle of the sublime, and of the terrible as anything beyond the familiar that is just as hard to imagine as is death itself. The Burkean sublime delight - the pleasure that "turns on pain" - is precisely the point of convergence between the urge for selfpreservation and the attraction of "society." It is the libidinal energy that runs between Eros and Thanatos. At the level of the body pain and pleasure are harder to reconcile, pointing to the paradoxical wishes of an ego that makes a game out of life and death as it struggles to coordinate its autonomy with its 'infantile' need for oneness with the rest of the world. Ultimately, the relationship between flow and Burke's physical sublime, and its extension in Freud and Bataille, demonstrates that human nature expresses itself through a dynamic relation to the world, in which action and passion do not stand in contradiction, self-affirmation tends toward self-denial, and the highest form of control is a kind of wilful surrender.

As I demonstrate the connection of "flow" to the concept of the sublime, Freud's primary drives, and Bataille's ecstasy, I confirm its suitability for the purposes of gamification. "Flow" is another concept that treats the problem of personal freedom as 
part of the individuation process of the self. For that reason, it successfully validates and signifies the mysterious power of the game as means of both empowerment and control. Thus, McGonigal's invitation to entrust our happiness to the expertise of "happiness engineers" and the addiction to games aligns well with Freud's observation that the uninhibited pursuit of pleasure is generally perceived as liberty. On the other hand, the overpowering effect of games that highjack the personal motivation and meaning making processes of the player can also be likened to erotic attraction or the life drive that threatens the integrity of the ego. In a similar fashion, Chatfield's reinterpretation of flow as a ceaseless and, yet, enjoyable demand on our attention and Hugos' "engagement engines" presuppose the same double bind of self-indulgence and enslavement. For Bataille this type of condition is an honest reflection of the divergent "potentialities of the human spirit". Submission to the attraction of fun and games is in line with his view that the course to sovereignty runs through transgression and excess. If the state of flow were achievable through gamification, and it had the quality of liberation achieved through controlled surrender, then gamification would have also constituted a possibility for transcendence.

Gamification's reference to the sublime through Csikszentmihalyi's notion of flow has several persuasive functions. On the one hand, it equates virtual and real experience by suggesting that sublime affect is possible in pre-designed, virtual environments. While the Kantian sublime referred to an affect produced by the abrupt presentation of external stimuli that had to be incorporated by consciousness, the concept of "optimal experience" or "flow" suggests that the effects of the sublime can be willed forth with the help of ready structures of engagement and mind techniques. Since the gamified subject thus 
becomes an instrument of oneself (and others) and is enclosed in a technological environment of its own making, its encounter with the sublime is also pre-designed. If 'flow' is the gamified sublime encounter, it follows that the imagination of the designer contains both the predictable and the unpredictable, both the imaginary as the interior of one's mind and the unimaginable as its ultimate challenge and exterior. For example, the user of Pymetrics may experience a form of sublime affect at the discovery of one's core traits if these match her own self-concept, and hence, are felt as a revelation. In this scenario, the user, at least momentarily equates the authority of the man-made algorithm to that of a higher power. Alternatively, if the user of Pymetrics is also the designer of the game algorithms, the games can only work properly on her if the designed algorithm continuously surpasses her design vision in unexpected ways. Paradoxically, then, the designer contains and designs the unpredictable without ever knowing it herself.

On the other hand, flow as a 'self-inflicted' sublime affect serves to validate the contradictory treatment of the gamified subject as both a means to an end and the source of an original intent. As it works through the conflicting desires of humans who want to be both separate and together, the notion reveals the underlining goal of the gamification project. It constructs an ideology that seeks to harmonize our claim to power with the need to belong, the individual desire for mastery with the comfortable predictability of structured group behaviour. In the context of Pymetrics, the coordination of opposing urges of the user translates into a consensual submission to the rules of the games or one's voluntary resignation from the responsibility of self-awareness and self-direction for the sake of access to one's own character blueprint and clues to one's best possible future. In 
other words, we submit to the authority of technology and accept the guidance of algorithms in order to purchase a "sense of mastery" over ourselves and our own fate.

While the meaning of flow in gamification discourse is mostly based on Csikszentmihalyi's conception of flow, it is not entirely identical with it. Csikszentmihalyi insists that the state of flow can be reached on the condition of voluntary investment of attention in an activity that is a purpose in itself. Yet, for Csikszentmihalyi flow is the ultimate purpose, while for gamifiers flow is the means to something else. As recognized by players, game theorists and designers, the experience of flow as an intense engagement and self-forgetfulness is a common occurrence in a video or computer game environments. In addition to the attractive visuals and elaborate plots, games still offer a refuge, a separation from the demands of real-life necessities and consequences. For that reason alone, the participation in a game is a matter of free choice and an end in itself. Conversely, in a gamified environment, where the game is defined as the means to an ulterior end, Csikszentmihalyi's conditions of flow are simply not met. If at all possible, the state of flow in a gamified environment will not only constitute a "self-inflicted" sublime affect, but will function as the sublime in reverse. It would be an experience in which the sense of exhilaration and mastery of the gamified subject precedes and facilitates the subject's subordination to the "ineffable", or at least unuttered purpose of the gamifier. Whether the state of flow is achievable in a gamified environment or not, the discourse and rhetoric of gamification promoters demonstrates their unprecedented ambition to design and utilize the sublime. 


\section{CONCLUSION}

Gamification claims to be a way of designing desirable experiences, and by extension, a way of evaluating, validating, designing ourselves. Its rhetoric and discourse outline the parameters of an ideological construct, a taken-for-granted real, where the self-esteem of humanity is expressed by an infinite faith in its technologies. It is the likely offspring of a techno-centrist ontology which respects the authority of the machine over that of the human.

If gamification rhetoric functions ideologically, it does so by constructing and addressing a subject. Therefore, in as much as this work has been about the rhetorical devices which legitimate gamification as a worldview, it has also been about the status of the gamified subject within a gamified environment. Gamifiers' adoption of Csikszentmihalyi's notion of 'flow' as a primary ground and aspiration is an attempt to reconcile the free-willed subject with the well-utilized one. On the one hand, the game part of gamification promises freedom, spontaneity and fun to a self-motivated agent. On the other hand, the craft of pre-designed experience develops and applies standard criteria for fun, desire, motivation, and thus produces a standardized subject, who is reliable and efficient. The ambiguity embedded in the concept of gamification is intended and 
functional. It persuades the gamified subject to submit to the mysterious logic of virtual environments and algorithms in order to gain access to the role of a master designer. The preceding chapters of this work reveal the levels of persuasion unfolding in the gamification narrative.

As my analysis of the texts of Hugos, Penenberg, McGonigal, and even Bogost demonstrates, the promotional rhetoric of gamification conflates the modern concept of the subject as rational and autonomous agent with the idea that subjective perceptions and affects can be designed and instrumentalized. Dramatistic analysis clearly exhibits this contradiction thorough series of changing pentadic relationships that construct the subject as both an agent and an agency, as the locus of the action and as the means to an end. In the texts of Hugos and Penenberg the variety of pentadic roles ascribed to the gamified subject are eventually coordinated into an hierarchy. Since utilitarian gamifiers are strictly concerned with the business application of games, the system of relationships they describe is comparatively straightforward. The order of participants reveals that the pentadic role of agent is strictly reserved for the designer or the gamifier, while the role of agency is ascribed to the gamified user. Games, or algorithms, play the important role of a mediator, which functions as agency (means) in respect to the gamifier, and as agent or scene (higher power, environment) in respect to the gamified user. According to Hugos, games motivate employees to work even harder towards the goals of the company. Their rhetorical and practical role is to make invisible the gamifier and her goals (ex: corporate owner) to the gamified (ex: employees). According to Penenberg, games are simulations which successfully disguise the pre-designed mechanical reactions of players as spontaneous choice of action. In both cases, however, the responsibility and intent of the 
true originator of the act of gamification is mystified through or dissolved into the "magical powers" of games and technology.

The hierarchical orders put forth by the texts of McGonigal and Bogost still place the gamifier in the leading agent position. As a humanitarian gamifier McGonigal proposes that gamification is not only a better instrument for organizing social interactions, but also a new mode of self-relation. In other words, the empowered gamified subject is the one who uses gamification on itself. Her proposition, constructed through an ambiguous entanglement of pentadic functions and ratio-transformations, normalises a logic of circular causality, according to which, consequences double as causes and ends trade places with the means. McGonigal's definition of the human being supports the idea that feelings and emotions, including happiness, can be reverseengineered and managed like other mechanical or chemical processes. However, the selfalienated, self-design perspective of oneself as both the gamified and the gamifier calls for a different kind of deception. This time games are needed as a mediator who obfuscates the consciousness of the self of itself as the designer. Self-deception through games is the only way an engineered emotion can be experienced as genuine.

Finally, Bogost, who positions himself as a theorist of games and gamification, also works within the already established hierarchical structure. His observation on the construction of meaning through effective word coinage offers the logological perspective on gamification rhetoric. However, the meta discussion he begins in his blog post is brought down to a detailed account of existing game applications in How to Do Things with Games. Here, by treating games as a medium Bogost invests them with the same kind of instrumentality that utilitarian gamifiers promote. Once again, the author's use of 
grammar demonstrates that the gamified subject is conditioned by the games, which, in turn, are used as means to further ends by the gamifier. Not unlike McGonigal, Bogost suggests that gamification can be empowering or understood as "performative play" when the player and the game designer coincide or the player freely accepts the conditions of the game. As a gamifier who applies games on itself to achieve certain experiences and emotions, the gamified subject is an agent who acts upon itself, uses itself as means, and is its own purpose. In other words, we use games and gamification to mediate and mystify the relationship of the self to itself.

The examined writers in chapter two agree that the real is insufficient without games or, at least, that games have a valid place as means for the production of real impact into non-game environments. Admittedly, gamification seeks to mould the real, design it, master it, upgrade it. However, the criteria it uses, and the goals it poses for itself, are undisclosed to the majority of users or participants in that real, and that seems to be a prerequisite for its success. Idealistically speaking, gamification works due to the "mysterious power" of games. Pragmatically speaking, it works thanks to the application of demystified principles of engagement which guarantee the reproducibility of desired effects. Whether this magical attraction to a gamified world is the result of voluntary suspense of disbelief or the masterful entrapment of good design, it would not work without a dose of deception or submission into an unknown. In that sense, Gamification seeks to add a layer of mystery onto the mundane, but ironically, it also seeks to make use of it, and control it. The idea of planned or designed mystery is the kind of paradox that points toward the inherent problems of the gamification attitude. 
If the promotional rhetoric of gamifiers like McGonigal and Hugos constructs a value system and a program of action, the applied gamification rhetoric used by Pymetrics acts according to it. Hence, the address of the Pymetrics website towards its users treats the subject as inaccessible to itself without the mediation of technology. This is a subject that has to accept and embody a subordinate position to that of algorithms and technology that validate, regulate, and authorise its self-understanding, desires and actions. The Pymetrics subject, however, is not simply knowable and acquiescent by means of technology, but it is continuously verifiable as a sign of itself. The content analysis of the Pymetrics website and neuroscience games captures the logic, according to which, the user submits to technology for the sake of self-discovery and better self-control only to be equated with and petrified into one's own digital trace. As a fact of contemporary life, the Pymetrics games and the social practices they initiate are the actualisation of Baudrillard's integral reality. Every claim Pymetrics makes, and function of algorithms it promotes, implies the exact translatability of one sign in another, as well as the equivalence of representation and experience. According to Baudrillard, one of the symptoms of virtuality or integral reality is that the sign is taken as the proof for the existence of its referent. The Pymetrics' Trait Report claims to be exactly that - the everlasting map and evidence of a person's cognitive blueprint, the guarantor of one's sincere coincidence with oneself. In this regard, the existence of Pymetrics, as a business practice and a cultural phenomenon, demonstrates that the gamification attitude is founded in and perpetuates integral reality as a value system and worldview. Integral reality, however, is also what Baudrillard considers the final destination of neo-liberal capitalism. It has been brought about by the signification logic of techno-scientific objectivism (i.e. "the code"), holds the 
aspirations of neo-liberal productivity and produces subjects that are ever more efficient, predictable and readily applicable as means or labour power.

As chapter four reveals, the complex psychology and motivation of the gamified subject is ultimately construed through the notion of flow. This is the notion used by gamifiers to address and coordinate the opposing tendencies towards mastery and submission on which gamification rests. Csikszentmihalyi's understanding that the sense of self is strengthened in moments of self-abandon can be aligned with familiar notions such as the Burkean and Kantian sublime, Freud's life and death drives, Bataille's eroticism. The commonality between these notions is the implicit problematization of freedom as the constant reverberation between control and surrender. The sublime in Kant is a self-affirming confrontation with the ineffable which brings the rational mind into awareness of itself. The Burkean sublime delight, the pleasure that "turns on pain", is another description of the individuation encounter that happens as a result of the clash between the urge for self-preservation and the attraction of "society." In turn, Freud treats the problem of human liberty as part of the ego formation process that coordinates the desire for self-preservation ("hunger", death drive) and the desire for belonging (Eros, life drive). For Bataille, individual sovereignty can only be attained through excess, whether that is excessive self-indulgence or excessive self-restraint. The ecstatic self emerges at the limit of its own annihilation, at the breaking point of the boundaries of the discontinuous being as it reaches towards a beyond. All of these thinkers, spanning the 18th to 20th centuries, refer to extreme states of being in order to comprehend the structure of self-consciousness or self-perception. In one form or another, all of them speak about the confrontation of this emergent, unified self with that which exceeds it, 
and in doing so, draw its horizon. However, in the context of gamification, the exteriority that confronts the self in the experience of flow or the sublime, is no other than predesigned and technological. Hence, the gamified sublime diverges from the traditional one by eliminating the other or the beyond from the formational encounter, and enclosing the self into itself as an absolute.

As a rhetorical gesture, the notion of "flow" is used by gamifiers to persuade that the entirety of experience can be technologically predicted, controlled and safe-guarded. The required faith in the impartial objectivity of algorithms is of the same nature as the faith in the chance operations of the dice. Baudrillard uses the metaphor of the dice to explain how conferring oneself to "objective randomness" is a tactic that seeks to relieve us from the responsibility of free will just as much as to protect us "against the unpredictable faithfulness of the world as it is" (2001, pp.61-62). "Objective randomness", however, is just as illusory as the impartiality of algorithms, because "nothing can come out of a computer which hasn't been programmed in, [and] nothing can come from the dice which hasn't already been programmed mentally" (Baudrillard, 2001, p.59). Thus, Baudrillard's dice is an early example of self-mediation technology, but also the epitome of the technological attitude that can conjure up gamification as the next attempt of "constructing an artificial destiny" or "programming the deregulation of life" (Baudrillard, 2001, p.62). The concepts of programmable chance or pre-designed mystery are perfect contradictions of terms that demonstrate the complex relationship between self and world. The technological effort for transcendence they constitute is doomed to failure, because our technologies may still be external to our bodies, but do not exceed our desires and imagination. 
Gamification rhetoric and discourse exude the techno-scientific enthusiasm that defines our time. If language mirrors the culture that produces it, then we live in a culture that equates human progress with the evolution of technology. The belief that mechanisms are necessarily more accurate and fair than humans verges on superstition. A number of thinkers have described the contemporary faith in the techno-scientific complex as a form of mythical thinking. In tracing the production of knowledge as ideology, Althusser finds the root of the problem in Spinoza's "theory of the difference between the imaginary and the true" (1970, p.17). Hence, he contends that "the knowledge effect of scientific knowledge" (Althusser, p.67) is the result of the nondistinction between the real object and the object of knowledge. In other words, like myth, ideological knowledge is "concerned with the real world through its specific mode of appropriation of the real world" (Althusser, 1970, p.54).

Working from within the same paradigm, Marcel O'Gorman (2015) is another author who emphasises the similarity between the social and cultural roles of myth and technology. He points out that "the technical aspect of the human has collided with the symbolic aspect, resulting in a situation where technicity itself has become the basis for heroic recognition" $(2015$, p.3). As O'Gorman explains, the myth making and "archival" functions of technology are evident in its fundamental motive. We use technology to develop strategies for the denial of human finitude and to achieve a sense of recognition without exposure to physical risk.

Vincent Mosco, on the other hand, underlies the connection between myth and technology by examining the meaning of cyberspace. He notes that "cyberspace has become the latest icon of the technological and electronic sublime, praised for its epochal 
and transcendent characteristics and demonized for the depth of the evil it can conjure" (Mosco, 2004, p.24). While he emphasizes the cultural significance of myth as a meaning-making and reality-shaping mechanism, he also draws attention to the political implications of the myths we make. As he details the construction of conventional truth, Mosco comments, "Myth can be viewed as an early step in a process that, when examined with a critical eye, can restore with every critical retelling a political grounding that myths appear to leave out $(2004$, p.16)". Undoubtedly, the ubiquitous belief in the authority of technology and science sustains a contemporary myth, which does not yet recognize and own its own political grounding. The gamification phenomenon itself is evidence that a world organized around the belief in scientific objectivity is not devoid of mystery and mystification.

The concept of gamification adds its own hue to the already vibrant myths constructed around technology. A strong indication of the ideological or myth-making functions of gamification rhetoric is its claim on our perception of reality. It promises to "fix" the triviality of the real by means of the imagination, or rather, imagined virtual means. By this suggestion, the realm of physical experience is not only mixed with that of the virtual, but is placed under its regulation. The myth of gamification extends two of the foundational values of neo-liberal capitalism: the freedom of the individual, and the belief that techno-scientific progress leads to better life. It constructs a narrative of promise that both imposes its hierarchy and represses consciousness of the inbuilt social inequality. Thus, once again, the neo-liberal or the gamified subject is coaxed by visions of personal grandeur in order to accept the role of resource or means. It is a myth built on ambiguity and contradiction, which naturalizes the higher authority of science and the machine. 
According to its instructions, the reward of self-understanding is earned by submitting oneself to the discerning skill of algorithms. The joy of intense engagement can be earned through submission to the addictive power of games. Self-mastery is purchased by delegating the responsibility of one's actions to the gamification app. Thus, the belief in the magic power of technology is necessarily supplanted by the internalisation of one's insufficiency in relation to it. More importantly still, the credibility of the technoscientific has to be guaranteed by its externalisation and the detachment from human intention, emotion and bodily sensation. We have estranged the body as the source of knowledge - a source, the technological way of knowing has also emerged from, but is now consistently alienating. The algorithmic knowledge of gamification eliminates the senses as the criteria of experience or as the measure of the real. It is the kind of knowledge which claims to have escaped the bias of human desire, but unavoidably remains the product of human imagination. Ironically, the human emotion that was the first to be sacrificed for the sake of 'scientific objectivity', is now the first candidate for reconstruction in the new machinic environment. Gamification constitutes the process of digitizing emotion in order to utilize it, but also ensuring its existence in a virtual realm and at a distance from the physical body. Thus, the technological instrument of knowledge has a curious effect when applied on oneself. To know myself technologically, I have to submit to the guidance and authority of the machine. Yet, to know myself technologically still remains a form of self-interpretation. This is how technology creates a magic circle of its own.

Finally, the important lesson that can be drawn from the observed likeness of mystical and techno-scientific thinking is not that both are unreliable and misleading. It 
would be more accurate to say that both myth and science are modes of representation. However, every representation is mythological, due to its metaphysical ambitions of organizing a world, and ideological, due to the implicit intentionality of its taken-forgranted truths. Embracing the mystery of technology is the same as believing in its neutrality. Both of these orientations work to distance the machine from the human who invents it, and allow the human purpose to reveal itself as a higher intent in the pretended absence of the human. From inception to application, technology is political, mythical, symbolic. It is an action with force and direction, a meaning-making, world-shaping manoeuvre. As Burke warns, "the dialectical approach to knowledge is through the act of assertion, whereby one "suffers" the kind of knowledge that is the reciprocal of his act" (1969, p.35). For this reason, technological inventions should not be seen as neutral and inevitable, but as an extension of our self-concept, as a mode of self-relation that does not need to be evaluated as accurate or inaccurate. Rather, it should be assessed as a motivated choice, as a beneficial or a detrimental human choice. 


\section{Bibliography}

Austin, J. L. (1975) How to do things with words. Second Edition, (Eds.) Urmson J. O. and Sbisà, M. Harvard University Press, Cambridge Massachusetts.

Althusser, L., \& Balibar, E. (1970). Reading "Capital". Ben Brewster (transl.) London: NLB.

Barthes, R. \& Lavers, A. (1987): Mythologies. New York: Hill \& Wang (1957c)

Bataille, G. (1962). Death and sensuality: A study of eroticism and the taboo.

New York:Walker and Company. [electronic resource]

Bataille, G., \& Dalwood, M. (2006). Eroticism. London: Boyars.

Baudrillard, J. (1975). The mirror of production. Mark Poster (transl.) St. Louis: Telos Press.

Baudrillard, J. (1981). For a critique of the political economy of the sign. St. Louis, MO: Telos Press. (First published in French 1972) . (1994). Simulacra and simulation. Ann Arbor: University of Michigan Press.

(Original work published 1981) . (1996). Cool memories II: 1987-1990. Durham: Duke University Pres. . (2001). Impossible exchange. London: VERSO . (2005). The intelligence of evil or the lucidity pact. Chris Turner (transl.)

Oxford: Berg.

Bogost, I. (2007) Persuasive Games: The Expressive Power of Videogames. Cambridge, Massachusetts: The MIT Press 
. (2011a) Gamification is Bullshit, [Online] Available at:

http://bogost.com/writing/blog/gamification_is_bullshit/ [Accessed 04 May 2013] . (2011b) How to do things with videogames. Minneapolis London: University of Minnesota Press. . (2016). Play anything: The pleasure of limits, the uses of boredom, and the secret of games. New York: Basic Books

Brinkmann, K. (2005). "Consciousness, self-consciousness, and the modern self", History of the Human Sciences, vol. 18.(4): 27-48.

[DOI: 10.1177/0952695105058469]

Brooker, P. (1999). A concise glossary of cultural theory. London: Arnold.

Burke, E. (1998) A Philosophical Enquiry into the Origin of our Ideas of the Sublime and Beautiful, ed. Adam Philips, Oxford and New York: Oxford University Press. (Copy of 6th ed. Dublin: Graisberry and Campbell, MDCCLXCI [1771]).

Burke, K. (1966). Language as symbolic action: Essays on life, literature, and method. Berkeley and Los Angeles: University of California Press. . (1969a). A grammar of motives. Berkeley: University of California Press. (1945c) . (1969b). A rhetoric of motives. Berkeley: University of California Press. $(1950 c)$ . (1985). "Dramatism and logology", Communication Quarterly, 33:2, 89-93, DOI: 10.1080/01463378509369584 [Online] Retrieved Dec 14, 2016 (http://dx.doi.org/10.1080/01463378509369584) 
Caillois, R. (2001). Man, play, and games. Meyer Barash (transl.) Urbana and Chicago: University of Illinois Press. (1st translation in English 1961)

Campbell, M. (Jan 5, 2011) "Game on: When work becomes play", New Scientist, ISSUE 2799 [Online] Available at:

(https://www.newscientist.com/article/mg20927940-300-game-on-when-workbecomes-play/) [Accessed 10 April 2013]

Castells, M. (2000). The rise of the network society (2nd ed.). Malden, Mass.: Blackwell Publishers.

Castronova, E. (2006). Synthetic worlds: The business and culture of online games. Chicago: University of Chicago Press.

Chatfield, T. (2010). Fun inc.: Why play is the 21st century's most serious business. London: Virgin.

Chou,Yu-Kai. (2017, February 7) "Top 10 eCommerce Gamification Examples that will Revolutionize Shopping" [Web log message] Retrieved from http://yukaichou.com/gamification-examples/top-10-ecommerce-gamificationexamples-revolutionize-shopping/

Coffey, P. (1999). The ethnographic self. London: Sage.

Consalvo, M. (2009). "There is no magic circle". Games and Culture, 4(4), 408-417. doi:10.1177/1555412009343575

Coonradt, C. (1985). The game of work: How to enjoy work as much as play. (1st ed.) Shadow Mountain. 
Constable, C. (2006) "Baudrillard reloaded: interrelating philosophy and film via The Matrix Trilogy." Screen 47, no. 2 (Summer 2006): 233-249. Communication \& Mass Media Complete, EBSCOhost (Accessed November 11, 2012).

Csikszentmihalyi, M., Halton, E. (1981).The Meaning of Things: Domestic Symbols and the Self . Cambridge: Cambridge University Press.

Csikszentmihalyi, M. (1997). Finding flow: The psychology of engagement with everyday life. (1st ed. ed.). New York: BasicBooks. . (2008). Flow: The psychology of optimal experience. New York: Harper Perennial. (c1990. 1st Harper Perennial Modern Classics ed.)

Crawford, C. (1997) The Art of Computer Game Design, Osborne/McGraw-Hill, Berkeley California. Print. (First published 1984)

Derrida, J. 'Speech on Différance', Margins of Philosophy, 1982, [electronic source]: www.hydra.umn.edu/derrida/diff.html, 15 - 16

Derrida, J., \& Anidjar, G. (2002). "Faith and Knowledge", Acts of religion. New York: Routledge.

Deterding, S., Dixon, D., Khaled, R., \& Nacke, L. (2011) "From game design elements to gamefulness: defining gamification". In Proceedings of the 15th International Academic MindTrek Conference: Envisioning Future Media Environments (September, 2011) pp. 9-15. ACM

Edery, D. Mollick, E. (2009) Changing the game: How video games are transforming the future of business. Pearson Education, Inc. publishing as FT Press 
Ellis, C. Adams, T. E. \& Bochner, A. P. "Autoethnography: An Overview", Forum Qualitative Social Research, Volume 12, No. 1, Art. 10 - January 2011 [Online] Available at: http://www.qualitativeresearch.net/index.php/fqs/article/view/ 1589/3095\#footnote_1 [Retrieved 30/03/2017]

Encheva, L. and Pedersen, I. (2014) ''One Day...': Google's Project Glass, integral reality and predictive advertising' Continuum: Journal of Media and Cultural Studies Vol. 28 - Issue 2: Postfeminist inflections in television studies, pp. $235-246$

Encheva, L. (2017) "The Gamified Sublime" in Trifonova, T. (ed.) Contemporary Visual Culture and the Sublime, Routledge: Taylor \& Francis Group

Fleming, D., and D. Sturm. (2011). Media, masculinities and the machine: F1, Transformers and fantasizing technology at its limits. New York: Continuum.

Freud, S. \& Strachey, J. (1961). Civilization and Its Discontents. New York: Norton. Goodman, Russell, "William James", The Stanford Encyclopedia of Philosophy (Summer 2017 Edition), Edward N. Zalta (ed.) [Accessed 8 July, 2017] $\mathrm{URL}=<$ https://plato.stanford.edu/archives/sum2017/entries/james/ $>$.

Grimes, M. Feenberg, A. "Rationalizing Play: A Critical Theory of Digital Gaming", (Re)Inventing the Internet: Critical Case Studies, A. Feenberg and N. Freisen (Eds), Sense Publishers, Rotterdam 2012

Gross, E. L. (2017, February 6) How This Founder Is Using Neuroscience To Help People Discover Their Dream Jobs. Forbes. Retrieved from https://www.forbes.com/sites/elanagross/2017/02/06/how-this-founder-is-usingneuroscience-to-help-people-discover-their-dream-job/\#5129f5541d16 
Halliday, M. A. K. (1978). Language as social semiotic: The social interpretation of language and meaning. London: Edward Arnold.

Hartley, G. (2003). The abyss of representation: Marxism and the postmodern sublime. Durham, NC: Duke UP.

Holt, N. L. (2003). Representation, legitimation, and autoethnography: An autoethnographic writing story. International Journal of Qualitative Methods, 2(1), 18-28.

Hugos, M. (2012) Enterprise games: Using game mechanics to build a better business. Sebastopol, CA : O'Reilly.

Huizinga, J. (2002). Homo ludens: A study of the play-element in culture. London: Routledge. (First published 1949)

Jenkins, H. (2006a). Convergence Culture: Where Old and New Media Collide. New York: New York University Press. . (2006b). Fans, Bloggers, and Gamers: Exploring Participatory Culture. New York: New York University Press.

Jensen, R. (1999). The dream society: How the coming shift from information to imagination will transform your business. New York: McGraw-Hill.

Kant, I. (2000) Critique of the Power of Judgment, (ed.) Paul Guyer, trans. Paul Guyer and Eric Mathews, Cambridge and New York: Cambridge University Press.

Kellner, D. "Jean Baudrillard", The Stanford Encyclopedia of Philosophy (Winter 2015 Edition), Edward N. Zalta (ed.), [Accessed on Jan. 2, 2014] URL $=<$ https://plato.stanford.edu/archives/win2015/entries/baudrillard/>. 
Koch, A. "Cyber Citizen or Cyborg Citizen: Baudrillard, Political Agency, and the Commons in Virtual Politics." Journal Of Mass Media Ethics 20, no. 2/3 (June 2005): 159-175. Communication \& Mass Media Complete, EBSCOhost (accessed November 11, 2012).

Koster, R. (2004). Theory of fun for game design. Scottsdale, AZ, USA: Paraglyph Press.

Lancelin, A. (July 2004). "The Matrix Decoded: Le Nouvel Observateur Interview with Jean Baudrillard", IJBS 1(2). Transl. Gary Genosko and Adam Bryx. [Online] Available at: (http://www.ubishops.ca/baudrillardstudies/vol1_2/genosko.htm)

Lapenta, F. "Geomedia: on location-based media, the changing status of collective image production and the emergence of social navigation systems." Visual Studies (March 2011), 26 (1), pg. 14-24

Lazzaro, Nicole. (June 3, 2010) "The Future of Work is Play", TEDxSoMa [Online] Available at: (http://www.youtube.com/watch?v=X_3KyV31iqg) [Accessed 6 June 2012]

Lipovetsky, G. (2005). Hypermodern Times. Cambridge: Polity Press.

Liyakasa, K. (May 2012). "Game On: Gamification Strategies Motivate Customer and Employee Behaviours" CRM Magazine, [Online] (Retrieved June 6, 2014) http://www.destinationcrm.com/Issue/3250-May-2012.htm [Access 6 June 2012]

Malone, T. W. (1980). What makes things fun to learn? A study of intrinsically motivating computer games. (Ph.D., Stanford University). ProQuest Dissertations and Theses, (303047451). 
Malone, T.W. \& Lepper, M.R. (1987) "Making learning fun: A taxonomy of intrinsic motivations for learning". In R.E. Snow and M.J. Farr (Eds.) Aptitude, Learning and Instruction III: Conative and Affective Process Analyses. Hillsdale, N.J.: Erlbaum.

Marx, K., Engels, F., In Arthur, C. J., \& Marx, K. (2004). The German ideology. New York: International Publishers. Print. 1972c

Maslow, A. H. (1970a). Motivation and personality. New York: Harper \& Row. . (1970b). Religions, values, and peak experiences. New York: Penguin.

\section{(1964c)}

McGonigal, J. (2011). Reality is broken: Why games make us better and how they can change the world. New York: Penguin Press.

Mosco, V. (2004). The digital sublime: Myth, power, and cyberspace. Cambridge, Mass: MIT Press.

Nancy, J. L. (1993). "Preface to the French Edition," Of the Sublime: Presence in Question, Jeffrey S. Librett (eds.), Albany, NY: SUNY Press.

Newton, E. (Oct 7, 2000). When work becomes play. The Vancouver Sun, pp. C.1.FRO-C1 / FRONT. Press.

O'Gorman, M. (2015). Necromedia. Minneapolis, MN : University of Minnesota Press Pedersen, I. (2013) Ready to wear: A rhetoric of wearable computers and realityshifting media. Parlor Press.

Penenberg, A. L. (2013) Play at work: how games inspire breakthrough thinking. New York : Portfolio/Penguin. 
Pink, D. H. (2009). Drive: The surprising truth about what motivates us. New York, NY: Riverhead Books.

Poster, M. (2001). What's the Matter with the Internet, University of Minnesota Press.

Reeves, B., \& Read, J. L. (2009). Total engagement: Using games and virtual worlds to change the way people work and businesses compete. Boston: Mass: Harvard Business Press

Tekinbaş, K. Salen., \& Zimmerman, E. (2003). Rules of play: Game design fundamentals. Cambridge, Mass: MIT Press.

Schell, J. (2008). The art of game design: A book of lenses. Amsterdam ; Boston: Elsevier/Morgan Kaufmann.

Schell, J. (2010). Jesse Schell: Visions of the Gamepocalypse. [Online] Available at: http://fora.tv/2010/07/27/Jesse_Schell_Visions_of_the_Gamepocalypse\#fullprogr am [Accessed 08 November 2016].

Schell, J. (2010). "The Future is Beautiful". TEDxUniPittsburgh [Online] Available at https://www.youtube.com/watch?v=0tg55pdNMxw [Accessed 08 Nov 2016]

Schell, J. (2011)."The Pleasure Revolution: Why Games Will Lead the Way". Google Tech Talk [Online] Retrieved: https://www.youtube.com/watch?v=4PkUgCiHuH8 [Accessed 08 Nov 2016]

Shinkle, E. (2012). "Videogames and the Digital Sublime", Digital cultures and the politics of emotion: feelings, affect and technological change, Karatzogianni, A. and Kuntsman, A. (eds.), Basingstoke: Palgrave Macmillan, 94-107 
Soja, E. W. (July 2001) "Digital communities, simcities and the hyperreality of everyday life." Lotus International no. 110 (July 2001): 73-87. Art Full Text (H.W. Wilson), EBSCOhost (accessed November 11, 2012).

Sparkes, A. C. (2000). Autoethnography and narratives of self: Reflections on criteria in action. Sociology of Sport Journal, 17, 21-41.

Stillar, G. (1998). Analyzing Everyday Texts: Discourse, Rhetoric, and Social Perspectives, Sage Publications: Thousand Oaks London New Delhi

Suits, B. (1978). Grasshopper: Games, life, and utopia. Buffalo: University of Toronto Press.

Sutton-Smith, B. (1997). The ambiguity of play. Cambridge, Mass.: Harvard University Press.

Taylor, T. L. (2006). Play between worlds: Exploring online game culture. Cambridge, Mass: MIT Press.

Thacker, E. (2001) "The Science Fiction of Technoscience: The Politics of Simulation and a Challenge for New Media Art". Leonardo. Vol. 34, No. 2 2001: 155-158.

Turner, C. (2005). "Foreword" to Baudrillard, J. The Intelligence of Evil and the Lucidity Pact. Oxford: Berg.

Turkle, S. (1995). Life on the screen: Identity in the age of the internet. Toronto: Simon $\&$ Schuster.

Wark, M. (2007). Gamer theory. Cambridge, Mass.: Harvard University Press.

Zichermann, G., \& Cunningham, C. (2011). Gamification by design. O'Reilly Media, Inc. Zichermann, G. (2010) "Fun is the Future: Mastering Gamification" GoogleTech Talks, (http://www.youtube.com/watch?v=6O1gNVeaE4g\&list=PL1B1A83D44CDBCA12) 


\section{Referenced company websites:}

Bunchball, Inc. ( )Home page. [Online] Retrieved from http://www.bunchball.com/), [Accessed 10 Nov, 2016].

The Games Institute, University of Waterloo. Home page [Online] Retrieved from https://uwaterloo.ca/games-institute/ [Accessed 24 June, 2017]

Genetics, Environment and Therapies: Food Allergy Clinical Tolerance Studies (GET FACTS) Project. Neil Randall, Ryan Clement, Kayla Marie Oliveira [Online] Available at: https://uwaterloo.ca/games-institute/projects\#GETFACTS. [Accessed 24 June, 2017]

Knack.it Corporation, Home page. Retrieved from: https://www.knack.it/ [Retrieved 23 June, 2017].

Lithium, Resources: "Drive the Right Customer Behaviours with Gamification!" (white paper) Retrieved from http://pages.lithium.com/gamification-spectrum.html), [10 Nov 2016]

Peer Doc: Gamifying a Professional Writing Collaboration Environment. Home page Retrieved from https://uwaterloo.ca/games-institute/Projects\#PeerDoc [June, 2017]

Pymetrics, Inc. (2013) Home page. Retrieved from https://www.pymetrics.com/ . Link: About Us. Retrieved from https://www.pymetrics.com/about/ . Link: Job Seekers. Retrieved from https://www.pymetrics.com/explore-

careers/ . Link: For Employers. Retrieved from https://www.pymetrics.com/enterprise/ 
. Link: The Science. Retrieved from https://www.pymetrics.com/the-science/ . Link: FAQ. Retrieved from https://www.pymetrics.com/faq/job-seeker/

Film:

Scott Card, O. (Producer) \& Hood, G. (Director). (2013). Ender's Game [Motion picture] United States: Summit Entertainment, Lionsgate Films

\section{Games:}

Brandstorm, L'Oréal. Reveal: The 2017 Challenge: Disrupt men's grooming with lifechanging innovation. Retrieved from http://www.brandstorm.loreal.com/en/challenges/play-experiment-innovate Enterprise Gamification Consultancy. (August, 2015) Trust by Danone, Retrieved from http://www.enterprise-gamification.com/mediawiki/index.php?title=Trust

Foursquare Labs, Inc. (2009) Foursquare. Retrieved from https://foursquare.com/

Habitica. (2015) Habitica: Motivate Yourself to Do Anything. Retrieved from https://habitica.com/static/front

Niantic. (2016) Pokémon Go. Retrieved from http://www.pokemongo.com/

Samsung. (2011) Samsung Nation. Retrieved from http://www.samsung.com/us/welcome_BV.html

Thales Group. (2008) Moonshield. Retrieved from https://www.thalesgroup.com/en/content/moonshield-discovering-thales-throughnew-online-game 